ANL-6499

Physics

(TID-4500, 17th Ed.)

AEC Research and

Development Report

ARGONNE NATIONAL LABORATORY

9700 South Cass Avenue

Argonne, Illinois

\title{
THE MEASUREMENT OF ENERGY AND INTENSITY \\ OF GAMMA RAYS BY USE OF A \\ SCINTILLATION SPECTROMETER
}

by

R. T. Julke, J。E. Monahan,

S. Raboy, and C. C. Trail

Physics Division

April 1962

Operated by The University of Chicago

under

Contract W-31-109-eng-38 


\section{DISCLAIMER}

This report was prepared as an account of work sponsored by an agency of the United States Government. Neither the United States Government nor any agency Thereof, nor any of their employees, makes any warranty, express or implied, or assumes any legal liability or responsibility for the accuracy, completeness, or usefulness of any information, apparatus, product, or process disclosed, or represents that its use would not infringe privately owned rights. Reference herein to any specific commercial product, process, or service by trade name, trademark, manufacturer, or otherwise does not necessarily constitute or imply its endorsement, recommendation, or favoring by the United States Government or any agency thereof. The views and opinions of authors expressed herein do not necessarily state or reflect those of the United States Government or any agency thereof. 


\section{DISCLAIMER}

Portions of this document may be illegible in electronic image products. Images are produced from the best available original document. 
ABSTRACT

I. INTRODUCTION 2

II. PROCEDURES FOR ANAL YSIS OF COMPIEX SPECTRA 6

Correction for Natural Background 14

Preparation for Analysis of Full-Energy Peaks: Program I 15

Preparation for Analysis of Full-Energy Peaks: Program II 20

Fitting Procedure: The Newton-Raphson Iteration Method 26

III. THE RESPONSE OF A SPEGTROMETER TO MONOENERGETIC GAMMA RAYS

Moment and Semi-invariant Generating Functions 32

a. Alternative events 34

b. Simultaneous events 34

c. Cascade events 35

Application to the Scintillation Counter $\quad 37$

a. Generation of light quanta in the scintillator 38

b. Collection of light quanta 39

c. Cascade of secondary electrons 41

Semi-invariants of the Pulse-height Distribution 41

The Response Function $\quad 44$

Maximum Likelihood Estimates of $\vec{a} \quad 46$

Probability Distribution of the Corrected Counts 52

Estimates Based on Corrected Spectra $\quad 54$

Summary $\quad 56$

IV. EXPERIMENTAL INVESTIGATION OF THE RESPONSE FUNCTION 58

The Line Shape $\quad 59$

The Line Width 62

The Energy Calibration of the Spectrometer: The Two-error Problem 
V. EXPERIMENTAL TESTS FOR CONSISTENCY

Measurement of Energy

Measurement of the Lifetimes of Radioactive Nuclei $\quad 74$

Discussion and Conclusions 82

APPENDIX. THE LEAST-SQUARES FORMALISM 83 


\begin{abstract}
The analysis of gamma-ray spectra measured with a scintillation counter with an anticoincidence annulus is described in detail. In particular the problem of the determination of the energy and intensity of gamma rays from such measurements is considered. The shapes of the full-energy peaks in observed complex spectra are studied and an attempt is made to justify their description in terms of a Gausian "response function." The details of the statistical analysis of complex gamma-ray spectra are given and, finally, procedures which have been used to confirm the validity of error estimates in energy and intensity measurements are described.
\end{abstract}




\section{INTRODUCTION}

This report deals mainly with the analysis of data taken with a. scintillation spectrometer with an anticoincidence annulus ${ }^{1}$ for the determination of energies and intensities of gamma rays. In an effort to utilize the full accuracy of this apparatus a program of analysis of observed spectra was developed for the digital computer GEORGE at the Argonne National Laboratory. The details of this method of data analysis as well as some experimental results which have been obtained by use of these methods are included in this report. The primary aim of this investigation is the establishment of realistic limits for the accuracy that can be attained with a crystal spectrometer in various energy and intensity measurements. The discussion begins with a brief description of the anticoincidence spectrometer.

The main detecting crystal is a cylinder of $\mathrm{NaI}(\mathrm{T} 1)$ about 2.4 in. in diameter and 6.0 in. long. It is optically coupled to a Dumont 6292 photomultiplier. If degraded photons resulting from Compton scattering or annihilation radiation escape from the center crystal, they are detected in a crystal annulus enveloping the main crystal. The annulus is a hollow cylinder, also of $\mathrm{NaI}(\mathrm{T} 1)$, with an 8-in, o.d., a 2.5-in, i.d, and a 12-in. length. Six photomultipliers are coupled to one end face of the annulus. In Fig. 1, we present an exploded view of this apparatus.

Signals from the center crystal are fed through a conventional linear amplifier to a multichannel pulse-height analyzer of the type designed by Schuman and McMahon. ${ }^{2}$ The six photomultipliers which are coupled to the annulus feed their signal through a common preamplifier and a linear amplifier to an anticoincidence gate of the analyzer. A pulse corresponding to a loss in energy of $30 \mathrm{kev}$ or more in the annulus is large enough to prevent the analysis of a pulse from the center crystal.

The spectra obtained with this anticoincidence spectrometer are characterized by the suppression of those contributions from Compton 


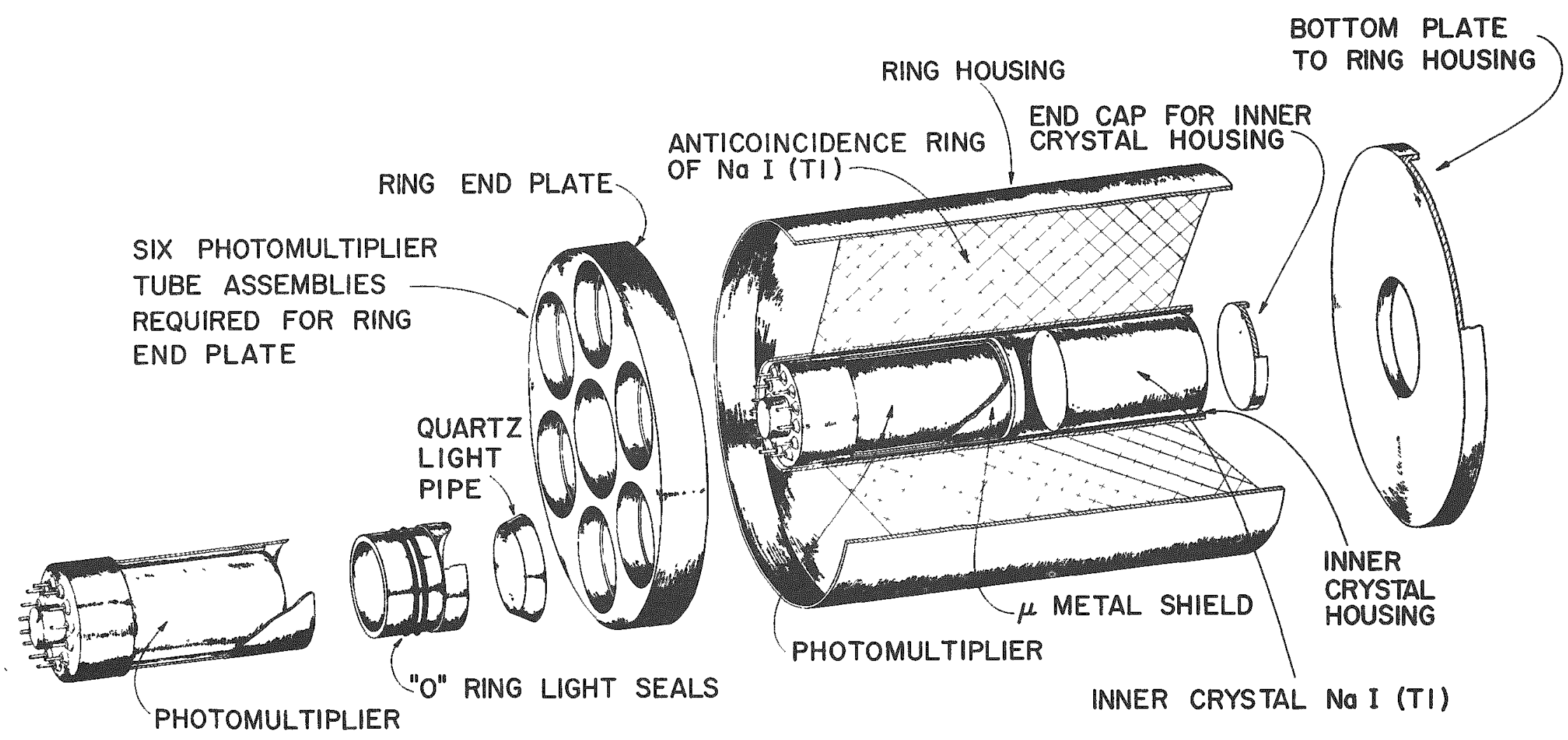

Fig. 1. An exploded view of scintillation spectrometer with an anticoincidence annulus. Signals from the outer crystal are placed in anticoincidence to those from the inner cylinder. 
and pair-production processes which do not contribute to the full energy peak. Thus the full energy peak of the gamma rays is apparently enhanced relative to the lower-energy portion of the spectrum. The improvement in the spectrum is illustrated in Fig. 2 which is a spectrum from a Na24 source.

The full-energy peaks of a "corrected spectrum" can be des cribed approximately by a Gaussian function and the essential feature of the analysis is the estimation of the values of the parameters that characterize this analytic function. (A "corrected spectrum" is one from which the con tributions from natural, or room, background and from other gamma rays have been subtracted in the neighborhoods of the full-energy peaks of interest.) The methods of obtaining such "corrected spectra" from observed spectra are discussed in Sec. II. Also in Sec. II is given the prescription for the determination of the parameters of the Gaussian function which approximates the full-energy peaks in such spectra.

Section III is devoted to the study of the response of the scintillation spectrometer, $i_{0} e$, to the derivation of the conditions necessary for an observed pulse-height distribution to approach a Gaussian form. The validity of this approximation for conditions which pertain in an actual experiment as well as the problem of calibration of the pulse height scale in terms of the energies of known gamma rays are described in Sec. IV. An experimental determination of the dependence of the widths of the line shape on the energy is also presented in Sec. IV.

In Sec. $V$ it is shown that the error estimates for the values of the parameters of the Gaussian function are realistic in two types of experiments, one is an experiment to determine the energy of a gamma ray and the other, an experiment to measure the intensity of a gamma ray from a radioactive source. These considerations indicate that within the interval from about $0.1 \mathrm{Mev}$ to 3.0 Mev energy measurements of about $0.1 \%$ accuracy are possible with this system and that measurements of relative intensity are a.ccurate to within about $0.5 \%$. 


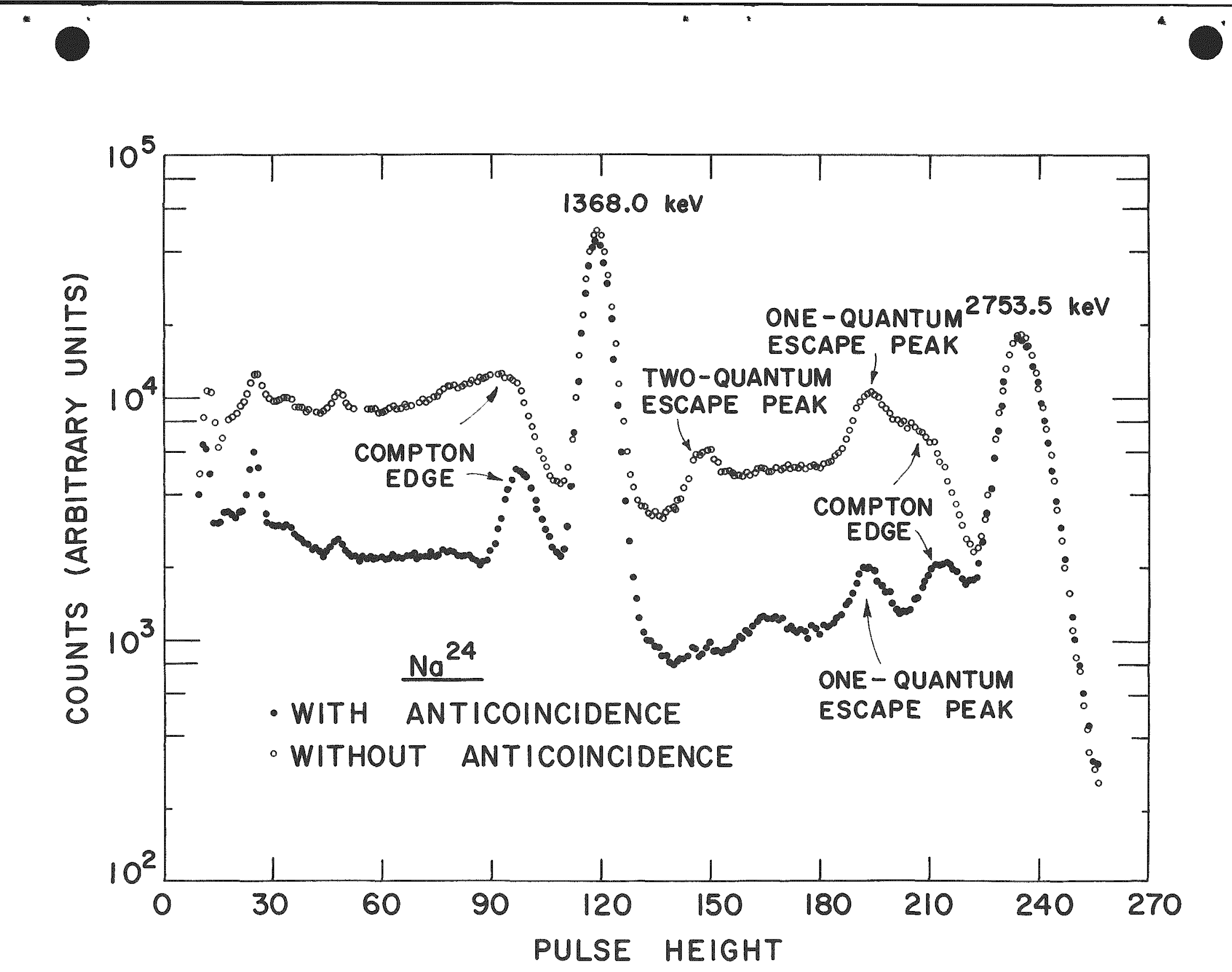

Fig. 2. Response of scintillation spectrometer to radioactive source $\mathrm{Na}^{24}$ to show advantage obtained by use of the anticoincidence system. 
The calibration of the pulse height scale in terms of gammaray energy is an example of a "multiple error" problem in statistical estimation. A least-squares formulation which is sufficiently general to include such problems is outlined in an appendix.

\section{ANALYSIS OF COMPLEX SPECTRA}

In a typical experiment, the observed gamma ray spectrum is a complex one which must be unscrambled in some way to yield quanti tative information about the nuclear parameters being studied. The analysis which is described in this section was developed for two specufic experiments, however, the methods are applicable to a wide range of experiments with the scintillation spectrometer.

In Fig. 3 is shown a spectrum of gamma rays from radioactive sources of $\mathrm{Na}_{24}, \mathrm{Ce} 144, \mathrm{Y}^{88}$ and Bi207. The results of this study have been published, but are included here as an example of the analytic method. The purpose of this experiment is to measure the energies of the gamma rays from Cel44 in terms of some "standards" from Na24, Y88, and Bi207. The spectra from all the sources must be accumulated simultaneously in order to reduce spurious effects such as variation of the gain with counting rate. As shown in Fig. 4, which is an abbreviated decay scheme of Ce144, the two gamma rays with lower energy are in cascade and the sum of their measured energies should equal, within expermental error, the measured energy of the crossover transition. This experiment, then, provides a quantitative check on the analytical procedures.

A second type of experiment which is of interest is the study of the variation in intensity of a gamma transition with some other parame. ter in the experiment such as time or energy of bombarding particle. In Fig. 5 two gamma-ray spectra from the $\mathrm{F}^{19}(\mathrm{p}$, a In this experiment the interest is in the variation in the yield of the 6. 1 , 6.9-, and 7.1-Mev gamma rays with proton energy. 
A.s will be shown in Sec. IV the response of a scintillation spectrometer to a source of monoenergetic gamma rays gives a full energy peak which to a good approximation is Gaussian in shape. However, even for this simple source the observed response exhibits a secondary peak from Compton processes in which the incident gamma ray is back-scattered and escapes through the entrance aperture of the spectrometer system. For such processes the energy remaining in the crystal gives rise to a peak which is lower in energy than the full-energy peak by the mean energy $\delta$ of the escaping quantum. This mean energy loss can be calculated in terms of the energy of the incident gamma ray and the geometric arrangement of the detector system. If the mean solid angle for such escape is sufficiently small, the response of the spectrometer to these Compton processes should also be Gaussian with an energy spread only slightly larger than the width of the full-energy peak.

These considerations lead to the postulate that the observed pulse-height spectrum near the full energy peak from a source of monoenergetic gamma rays can be described in terms of a set of functions of the form

$$
F_{j}(\vec{a})=\int_{\Delta_{j}} d x f(x ; \vec{a}), j=1,2, \ldots, N
$$

where

$$
f(x ; \vec{a})=a_{1} \exp \left[-\frac{x-a_{2}}{a_{3}}: a_{4}^{2} \exp \left[-\left(\frac{x-a_{2}+\delta}{k a_{3}}\right)^{2}\right]+a_{5}\right.
$$

In these expressions $F_{j}(\vec{a})$ represents the number of counts in the jth channel of the pulse-height analyzer, $\Delta_{j}$ is the width of the jth channel, $\delta$ is the mean energy carried off by the degraded photon in a Compton process as discussed in the preceding paragraph, $k$ is an input parameter which corrects for the difference in width of the second peak, and $\vec{a}$ is a vector with five components which are the parameters of the response function (II. 2). If $\Delta_{j}$ is defined to 
Fig. 3. Response of system to simultaneous exposure to a source containing several different activities and also the response to the corresponding isolated sources. Full scale corresponds to about 3 Mev。

a. Response of scintillation spectrometer to the gamma rays of $\mathrm{Na}^{24}, \mathrm{Ce}^{144}, \mathrm{Y}^{88}$, and $\mathrm{Bi} 207$.

b. Spectrum of isolated source of $\mathrm{Na} 24$

c. Spectrum of isolated source of Cel44

d. Spectrum of isolated source of $Y^{88}$

e. Spectrum of isolated source of Biz07

f. Spectrum of isolated source of $\mathrm{Zn}^{65}$. This spectrum was used to simulate the gamma ray at $1.06 \mathrm{MeV}$ of $\mathrm{Bi} 207$

g. Room background accumulated in a length of time about 10 times longer than required to accumulate the data for the other spectra. 


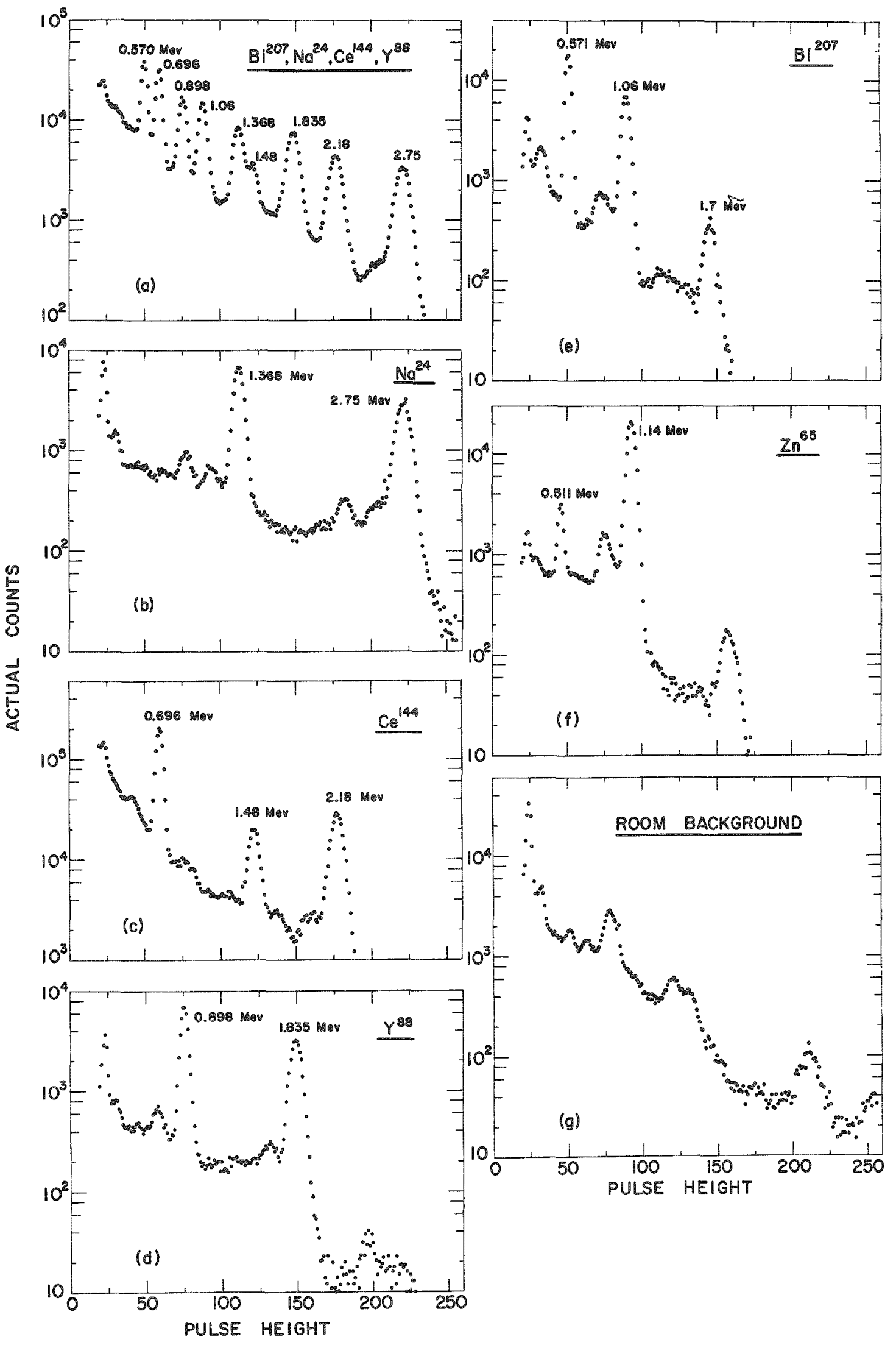




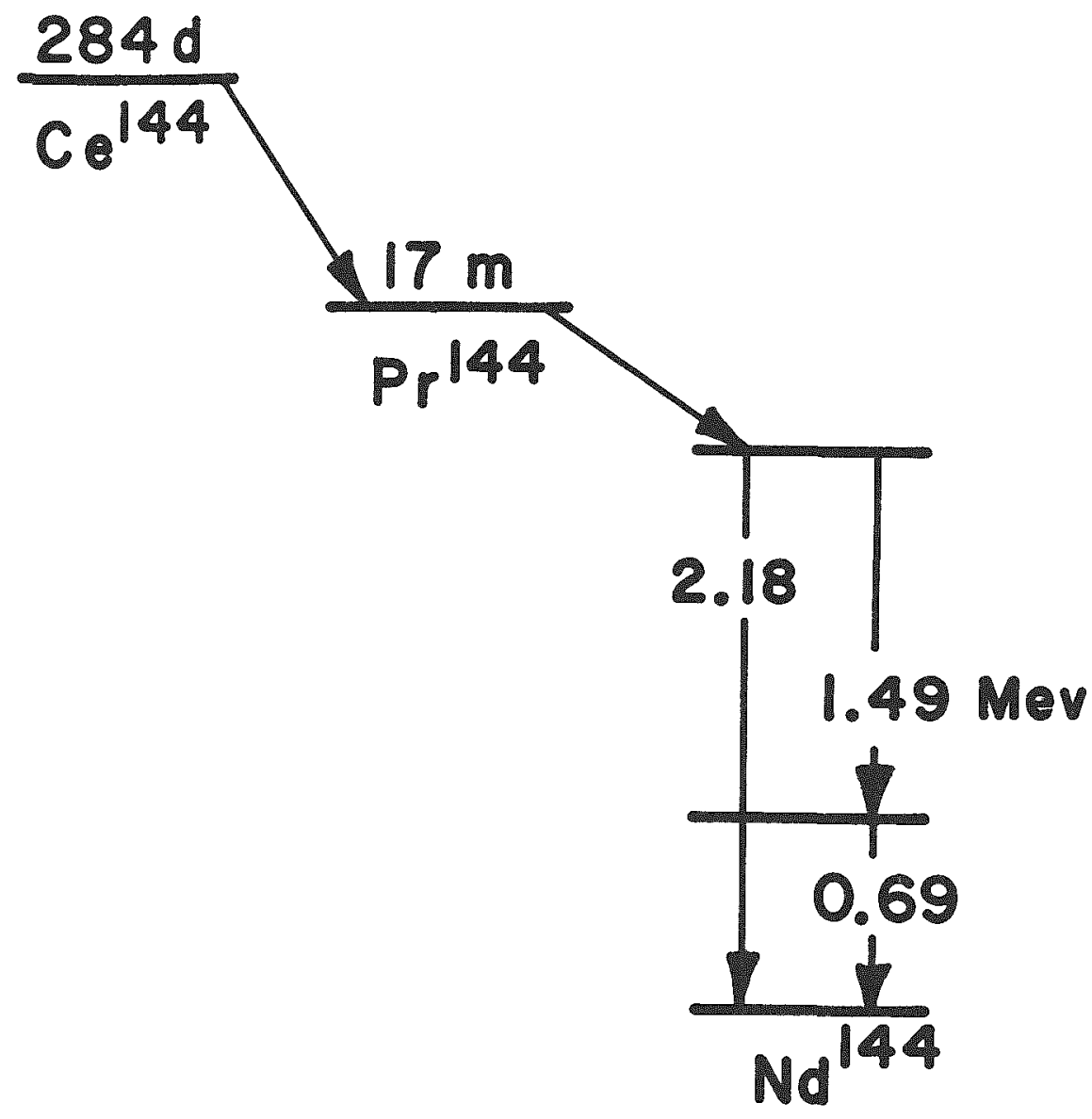

Fig. 4. Partial decay scheme of Cel44. 


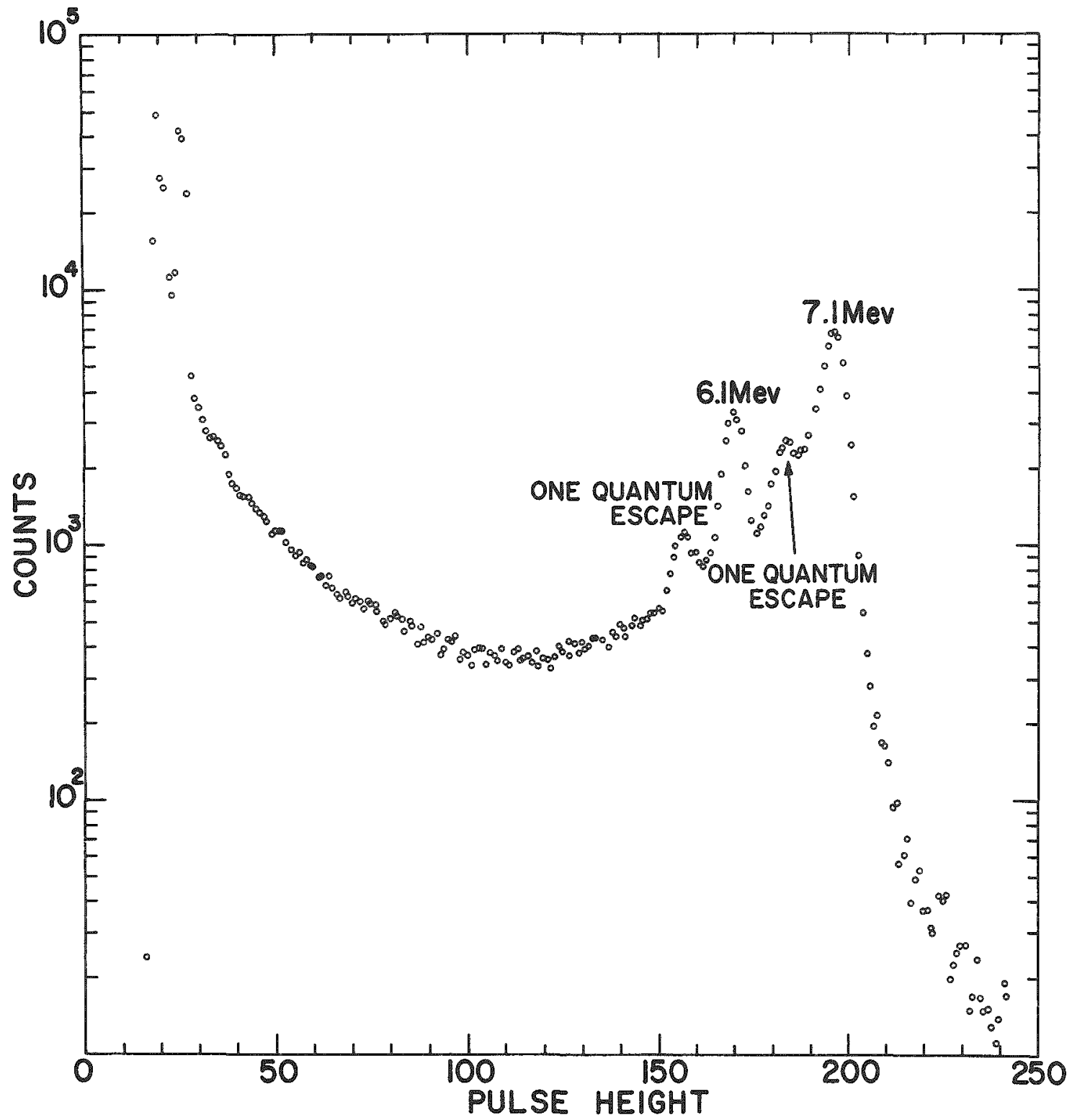

Fig. 5. Spectra of gamma rays from F19(p. ay)O1E. Energy of bombarding proton is $1.387 \mathrm{Mev}$. 
be the interval from $j-\frac{1}{2}$ to $j+\frac{1}{2}$, then $a_{2}$ and $a_{3}$ are respectively the mean position and width (in units of channel number) of the full-energy peak, $a_{1} a_{3}$ is proportional to the axea of the full-energy peak, $a_{4} a_{3}$ is proportional to the area of the back-scattered peak, and $a_{5}$ is a constant background.

Consider now the measured spectrum from a source of monoenergetic gamma rays. The spectrum is represented by a set of counts $c_{j} \Delta_{j}, j=1,2, \ldots, N$, where $\Delta_{j}$ is the channel interval defined above and $c_{j}$ is the average value in the channel of the number of counts per unit pulse-height interval. From the data it is possible to obtain a "best" estimate of the vector $\vec{a}$ and of the corresponding set of response functions $E_{j}(\vec{a})$. For the present a "best" estimate of $\vec{a}$ is defined as that vector for which the function $Q(\vec{a})$ is a minimum, where

$$
Q(\vec{a})=\sum_{j} \Lambda_{j}^{2}\left[c_{j} \Delta_{j}-F_{j}(\vec{a})\right]^{2}
$$

The $\Lambda_{j}^{2}$ are weighting factors determined from the counting statistics of the observed spectrum as specified in Sec. III. The rationale of this def1. nition is also discussed in Sec. III.

The analysis discussed above is applicable only to the case of a spectrum which results from a single source of monoenergetic gamma rays. In what follows, this type of spectrum will be designated as a "simple spectrum" and a spectrum that includes gamma rays of more than one energy will be called a "complex spectrum." For complex spectra it is necessary to modify the above approach. To effect this modification we attempt to isolate a set of equivalent simple spectra from a measured complex spectrum by subtracting from the measured spectrum all contributions other than that from the particular gamma ray of interest. This leads to a consideration of the background in the neighborhood of a particular full-energy peak that results from the presence of gamma rays of different energy in the measured spectrum. 
As long as the separation between photopeaks in the complex spectrum is much larger than the instrumental line widths, photopeaks at lower energies contribute negligibly to the background in the neighborhood of a given full-energy peak. However, because of incomplete suppression of Compton and pair processes by the anticoincidence feature of the spectrometer, there can be an appreciable contribution in the neighborhood of the photopeak from gamma rays of higher energy. Thus, at least in the case of gamma rays whose energies are well separated, we need consider only the contribution from higher-energy gamma rays in any estimation of background in the neighborhood of a given full-energy peak。

In many measurements, the observed complex spectrum results from several different radioactive sources so that it is possible to measure the component simple spectra separately. For complex spectra resulting from many transitions of a single nuclear species, the component simple spectra may be simulated, in special circumstances, by sources of monoenergetic gamma rays with appropriate energy. On the other hand, there are complex sources for which this is not feasible. The subtraction of background due to the "tails" of other gamma rays is considerably less arbitrary in those cases where such auxiliary measure. ments are possible. For this reason two subtraction procedures are used. The first one takes advantage of the additional information provided by the measurement of the component spectra and differs from the procedure used to analyze spectra for which no such auxiliary measurements are possible. The details of these methods are described in this section as Programs I and II. First, however, the correction of a measured spectrum for natural background will be considered.

It is always possible to determine the natural (or room) background spectrum in an obvious manner. It seems desirable, therefore, to include the subtraction of this background in any data-preparation procedure so that the resulting isolated lines, $i_{0} e_{0}$, the component simple spectra, should be approximated by the functions $F_{j}(\vec{a})$ in $E q$. (II. 1) with $a_{5}$ approximately equal to zero. 


\section{Correction for Natural Background}

Although these subtraction procedures are simple and

straightforward, the details are quite tedious and their exposition necessitates an inordinate amount of symbology which we now introduce. Many of the definitions are given in terms of sets. However, this is merely a convenient notational device and no use whatever is made of any settheoretical operations.

Let the observed (complex) spectrum consist of a set of counts $c_{j} \Delta_{j} ; i_{0} e_{0}$,

$$
Q=\left\{c_{j} \Delta_{j}: j=1,2, \ldots, N\right\} .
$$

The corresponding room-background spectrum will be denoted by , where

$$
\mathscr{C l a}_{\mathrm{a}, \mathrm{j}}=\left\{\mathrm{b}_{\mathrm{j}} \Delta_{\mathrm{j}} \mathrm{j}=1,2, \ldots, \mathrm{N}\right\}
$$

If $\mathrm{T}_{\mathrm{c}}$ and $\mathrm{T}_{\mathrm{b}}$ denote the counting times for the measurements $P$ and respectively then the spectrum corrected for room background is the set

$$
\left\{c_{j} \Delta_{j}-T_{c} b_{j !} \Delta_{j} / T_{b^{\circ}} j=1, \ldots, N\right\}
$$

where for each index $j$ the corresponding value $j^{\prime}$ is to be determined such that $j$ and $j$ 'refer to the same gamma-ray energy and $\Delta_{j}$ and $\Delta_{j}$ refer to the same interval in energy. The possible inequality between $j$ and $j^{\prime}$ and between $\Delta_{j}$ and $\Delta_{j}$, can arise because of slight changes in gain of the am plifier or of the photomultiplier tube in the time interval between the measurements $\odot$ and $\boldsymbol{B}$.

It is possible to insure experimentally to a good approximation against a relative displacement of the zero position of the two spectra and thus to maintain a constant value for the ratio $j^{\prime} / \mathrm{j}$ for changes in the 
gain of the system. A measure of the value of this ratio can be obtained as follows: Spectrum is also evident in 2 . This structure provides a means of calibrating the gain of the spectrometer. In the event such structure is not present, this calibration can be provided with a radioactive source before and after the measurements of $Q$ and $k$. Suppose such a calibration, or "mark," exists in 2 at $j=\widetilde{j}$ and in $\mathcal{K}^{\prime}$ at $j^{\prime}=\widetilde{j}+$ p. Since these "marks" correspond to the same energy it follows that

$$
j^{\prime}=j(1+p / \widetilde{j})
$$

and the spectrum corrected for room background consists of a set of elements of the form

$$
\bar{c}_{j} \Delta_{j}=\left[c_{j}-T_{c}(1+p / \widetilde{j}) b_{j^{\prime}} / T_{b}\right] \Delta_{j^{\prime}} j=1, \ldots, N
$$

Since $j^{\prime}$ is not necessarily an integer, an interpolation formula is used for the evaluation of $\mathrm{b}_{j}$, in Eq. (II.5). This is given as

$$
b_{j^{\prime}}=b_{\lambda}+\left(b_{\lambda+1}-b_{\lambda}\right)\left(j^{\prime}-\lambda\right), \quad \lambda<j^{\prime}<\lambda+1
$$

where $\lambda$ is an integer and $j^{\prime}$ is related to the integer $j$ by Eq. (II. 4).

\section{Preparation for Analysis of Full-Energy Peak: Program I}

We now consider the subtraction of the degraded radiation from higher energy gamma rays in those cases in which all of the component simple spectra of the complex spectrum have been measured separately, In this program, the possibility is neglected of perturbations by its one-quantum escape peak on the photopeak being analyzed. This approximation is permissible for gamma-ray energies less than about $3 \mathrm{Mev}$ 
since for the se energies the one-quantum escape peak is well resolved from the photopeak. Also at these energies the cross sections in NaI for the creation of pairs is relatively small. The modifications necessary for the inclusion of these processes are considered in Program II.

Let the complex spectrum $C$ consist of $r$ full-energy peaks and let the parameter vector (i.e., the vector for which $Q$ in Eq. (II.3) is a minimum) for the ith line in $C$ be denoted by $\vec{\beta}^{(i)}$. The measured simple spectrum for the ith line in $\Theta$ is denoted by $\Theta^{(i)}$ and the corresponding parameter vector by $\overrightarrow{\mathbf{a}}(i)$. The determination of the values of the components of the $r$ vectors $\vec{\beta}^{(i)}$ is, of course, the goal of this analysis. Let the $r$ lines in $C$ be numbered in order of decreasing energy such that

$$
\beta_{2}^{(1)}>\beta_{2}{ }^{(2)}>\cdots>\beta_{2}{ }^{(x)} \text {. }
$$

where $\beta_{2}{ }^{(i)}$ is component no. 2 of $\vec{\beta}^{(i)}$, and let the measured spectrum $C^{(i)}$ whose full-energy peak is centered at $a_{2}{ }^{(i)}$ correspond to the fullenergy peak in $C$ centered at $\beta_{2}{ }^{(i)}$. Reference to Fig。 3 may be helpful at this point.

Consider first the subtraction from the line in $Q$ at $\beta_{2}{ }^{(2)}$ of the tail of the higher energy line at $\beta_{2}{ }^{(1)}$. In order to carry out this subtraction the spectrum $\bigodot^{(1)}$ is first normalized to the intensity of $\Theta$ in the neighborhood of $\beta_{2}^{(1)}$ by multiplying each element in $\varrho^{(1)}$ by the

TIf the sources for the simple spectra have lifetimes long compared with the time interval for their measurements, the correction for the influences of these various peaks can be made in the manner described above for natural background. However, if these sources decay appreciably during the course of the experiment, these subtraction procedures must be modified to allow for the normalization of the areas of the photopeaks in the simple spectra to the areas of the corresponding photopeaks in the complex spectra. 
ratio of the intensities of the two spectra; $i_{0} e_{0}$, by the factor $N^{(I)}$, where

$$
N^{(1)}=\beta_{1}^{(1)} \beta_{3}^{(1)} / a_{1}^{(1)} \alpha_{3}^{(1)}
$$

(Since the superscript (1) refers to the highest energy line in $P$, there is no subtraction of the tails of higher energy lines in the neighborhood of this peak and values of the components of $\vec{\beta}^{(1)}$ can be obtained in the same manner as in the case of a simple spectrum.) The contribution from the tail of the line centered at $\beta_{2}^{(1)}$ to the line centered at $\beta_{2}^{(2)}$ in $\sigma_{-}$is the corresponding tail in $\mathrm{O}^{(3)}$ multiplied by $\mathrm{N}^{(1)}$. Thus in the neighborhood of $\beta_{z}{ }^{(2)}$ the spectrum corrected for the influence of the higher energy line at $\beta_{2}{ }^{(1)}$ is

$$
c_{j} \Delta_{j}-N^{(1)} c_{j !}^{(1)} \Delta_{j 1}
$$

where $c_{j} \Delta_{j}$ is an element of $\bigcirc$ and $c_{j^{\beta}}{ }^{(1)} \Delta_{j !}$ is the corresponding element in $C^{(1)^{j}}$. Again a distinction between $j$ and $j^{\prime}$ is made to allow for drifts and changes in gain during the interval of time between the measurements $\Theta$ and $C^{(1)}$.

To a good approximation, the electronic apparatus can be adjusted to make the pulse height of the full energy peak proportional to the energy of the incident gamma ray. For purposes of background subtraction, therefore, this proportionality is a valid assumption. The condition then that $j$ and $j$ ' refer to the same energy is

$$
j=k j^{\prime} \text { 。 }
$$

where, since $a_{2}^{(2)}$ and $\beta_{2}^{(2)}$ refer to the same gamma-ray energy

$$
k=\beta_{2}^{(1)} / a_{2}^{(1)}
$$


Expression (II.7) can be rewritten as

$$
\overline{c_{j} \Delta_{j}}=\left[c_{j}-\beta_{I}{ }^{(1)} \beta_{3}{ }^{(1)} a_{2}{ }^{(I)} c_{j}{ }^{(I)} /\left(a_{1}{ }^{(I)} a_{3}{ }^{(I)} \beta_{2}{ }^{(I)}\right)\right] \Delta_{j},
$$

where

$$
j^{\prime}=a_{e}^{(1)} j / \beta_{2}^{(1)}
$$

Again, since $j^{\prime}$ is not necessarily an integer, the value of $c_{j \prime}\left({ }^{\prime}\right)$ is calculated from the formula

$$
c_{j^{\prime}}^{(1)}=c_{\lambda}^{(1)}+\left(c_{\lambda+1}{ }^{(1)}-c_{\lambda}^{(1)}\right)\left(j^{\prime}-\lambda\right), \quad \lambda<j^{\prime}<\lambda+1,
$$

where $\lambda$ is an integer.

In order to facilitate the remainder of this discussion we make the following definitions:

$$
\mathcal{Y}\left(\underline{j}_{2} \bar{j}: C \backslash B\right) \equiv\left\{\bar{c}_{j} \Delta_{j}, c_{a} \Delta_{a}: \underline{j} \leqslant j \leqslant \bar{j} ; i \leqslant a \leqslant N, a \neq j\right\}
$$

where $\bar{c}_{j} \Delta_{j}$ is defined by Eq. (II.5) and $c_{a} \Delta a$ is an uncorrected element in $Q_{\text {. This set }}$ then is the spectrum $e_{\text {corrected for room background }}^{a}$ $B$ in the closed interval $[j, \bar{j}]$.

$$
\not\left(j_{2} \bar{j}_{0} \varrho \oint^{(i)}\right) \equiv\left\{\bar{c}_{j} \Delta_{j}, c_{a} \Delta_{a}: j \leqslant j \leqslant \bar{j} ; 1 \leqslant a \leqslant N, a \neq j\right\}
$$

where $\bar{c}{ }_{j} \Delta_{j}$ is defined by Eq. (II.8) with $c_{j} \Delta_{j}$ in $\varrho_{\text {and } c_{j}{ }^{(i)} \Delta_{j !} \text { in }} C^{(i)}$. Again $c_{a} \Delta_{a}$ is an uncorrected element in $\varrho^{j}$. The set $\mathcal{H}$ denotes the complex spectrum $Q$ corrected in the closed interval $[j, \bar{j}]$ in the neighborhood of $\beta_{2}{ }^{(i+1)}$ for the tail of the peak at $\beta_{2}{ }^{(i)}$. 
We are now in a position to outline the sequence of steps by which the vectors $\vec{\beta}^{(i)}$ are obtained from a set of measurements con-

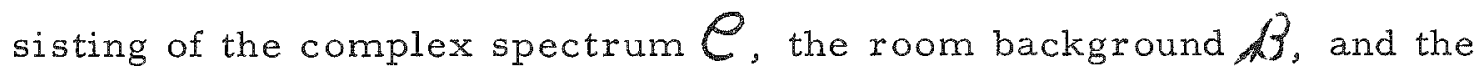
family of simple spectra $O(i)$. Recall that the $x$ lines in $C$ are numbered in order of decreasing energy and that the isolated spectrum corresponding to the ith line in $C_{\text {is }}(i)$. The steps of the subtraction procedure are as follows: (1) The spectrum $C_{\text {is corrected for room background }}$ and the resulting spectrum, namely $Y\left(j, j_{0}: C, B\right)$, where $[j, \bar{j}]$ is a predetermined interval about $\beta_{2}{ }^{(1)}$, is used to estimate the values of the components of $\vec{\beta}^{(1)}$ in such a way that they will satisfy the conditions associated with Eq. (II. 3). The summation in Eq. (II.3) is to be taken over the interval $[j, \bar{j}]$. (2) The isolated spectrum $Q^{(I)}$ is corrected for room background and the resulting spectrum $\left.Y\left(y_{0}, \alpha^{1}\right), \beta\right)$ is used to estimate the values of the components of $\vec{a}^{(1)}$. (3) The influence of the gamma ray of highest energy is subtracted from the entire spectrum 0 give

$$
e^{\prime} \equiv \Delta\left(1, N: e e^{(1)^{\prime}}\right)
$$

where

$$
Q^{(1)^{\prime}} \equiv Y\left(1, N: O^{(1)} \not B\right)
$$

The set of elements, $P$, represents the original complex spectrum with all influence of the highest energy gamma ray subtracted out. Thus $O_{1}$ is a complex spectrum whose highest energy peak is that centered at $\beta_{2}{ }^{(2)}$ and it is possible to analyze this spectrum to obtain the components of $\vec{\beta}^{(2)}$ in exactly the same manner in which $\vec{\beta}^{(1)}$ was obtained from $C$. Obviously this closed cycle of steps can be used to exhaust the lines in $\bigcirc$ and so to obtain the entire family of parameter vectors $\vec{\beta}$ (i). 
Preparation for Analysis of Full Energy Peaks: Program II

We again consider a complex spectrum $P$ consisting of $r$ full-energy peaks with associated parameter vectors $\vec{\beta}$ (i). However, we no longer assume that the complete family of component simple spectra have been measured, nor do we assume that all full-energy peaks in $e$ are sufficiently well separated that there is negligible contribution in the vicinity of a given peak from a neighboring peak at lower energy。

Originally this program was formulated to analyze photopeaks associated with gamma rays with energies in the neighborhood of $7 \mathrm{Mev}$ from the $\mathrm{F}^{19}\left(\mathrm{p}, \mathrm{a}_{\gamma}\right) \mathrm{O}$ reaction. Some typical spectra are shown in Figs. 5, 6, and 7. At these energies two additional complications arise. First, the one-quantum escape processes associated with each full-energy line are not negligible; and second, the back-scattered events leave enough energy in the crystal to distort the low energy side of the full-energy peak. Both of these possibilities are considered in the present formulation。

Let $\operatorname{Bdenote}$ the room background spectrum associated with the measurement $\varrho$ and consider the set, $1\left(\underline{j}, \bar{j} ; C_{1}(3)\right.$ where the interval $[j, \bar{j}]$ includes both $\beta_{2}{ }^{(1)}$ and $\beta_{2}{ }^{(2)}$. Within this interval there may be a non-negligible contribution from the four peaks representing the back-scattered and one-quantum escape events associated with both fullenergy peaks. We assume that, for each channel $j$ included in $[j, \bar{j}]$, the contribution to the number of counts due to the se secondary events can be approximated by four Gaussians which are given by an expression of the form

$$
d_{j}=\sum_{k=1}^{4} A_{k} \exp \left[-\left(\frac{j-j_{k}}{\sigma_{k}}\right)^{2}\right] .
$$

Since the one-quantum escape peak is $511 \mathrm{kev}$ lower in energy than its as sociated full-energy peak and the back-scattered peak is lower in energy 


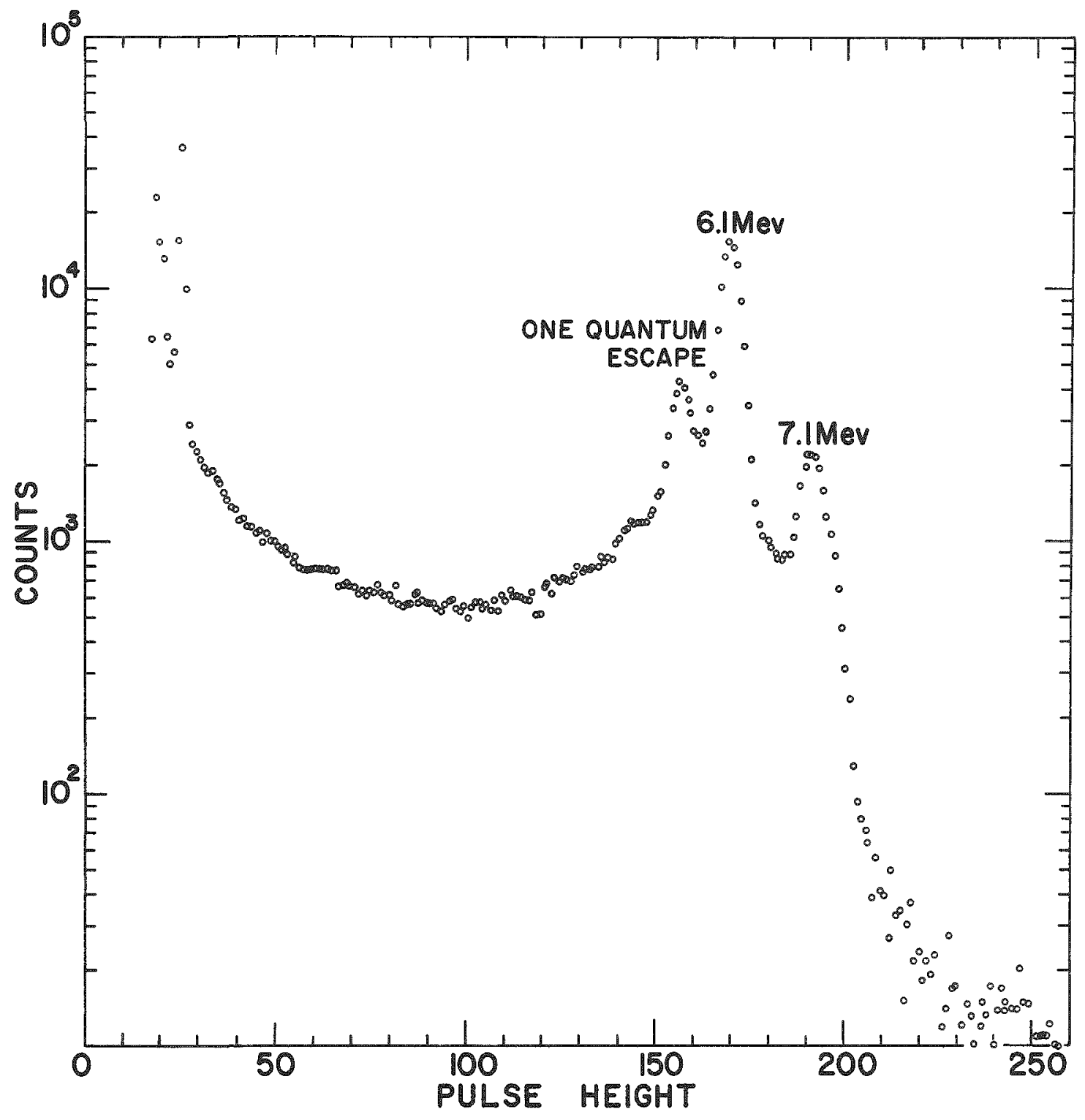

Fig. 6. Gamma rays from F19(p, ay)O1s. Energy of bombarding proton is $1.868 \mathrm{Mev}$. 


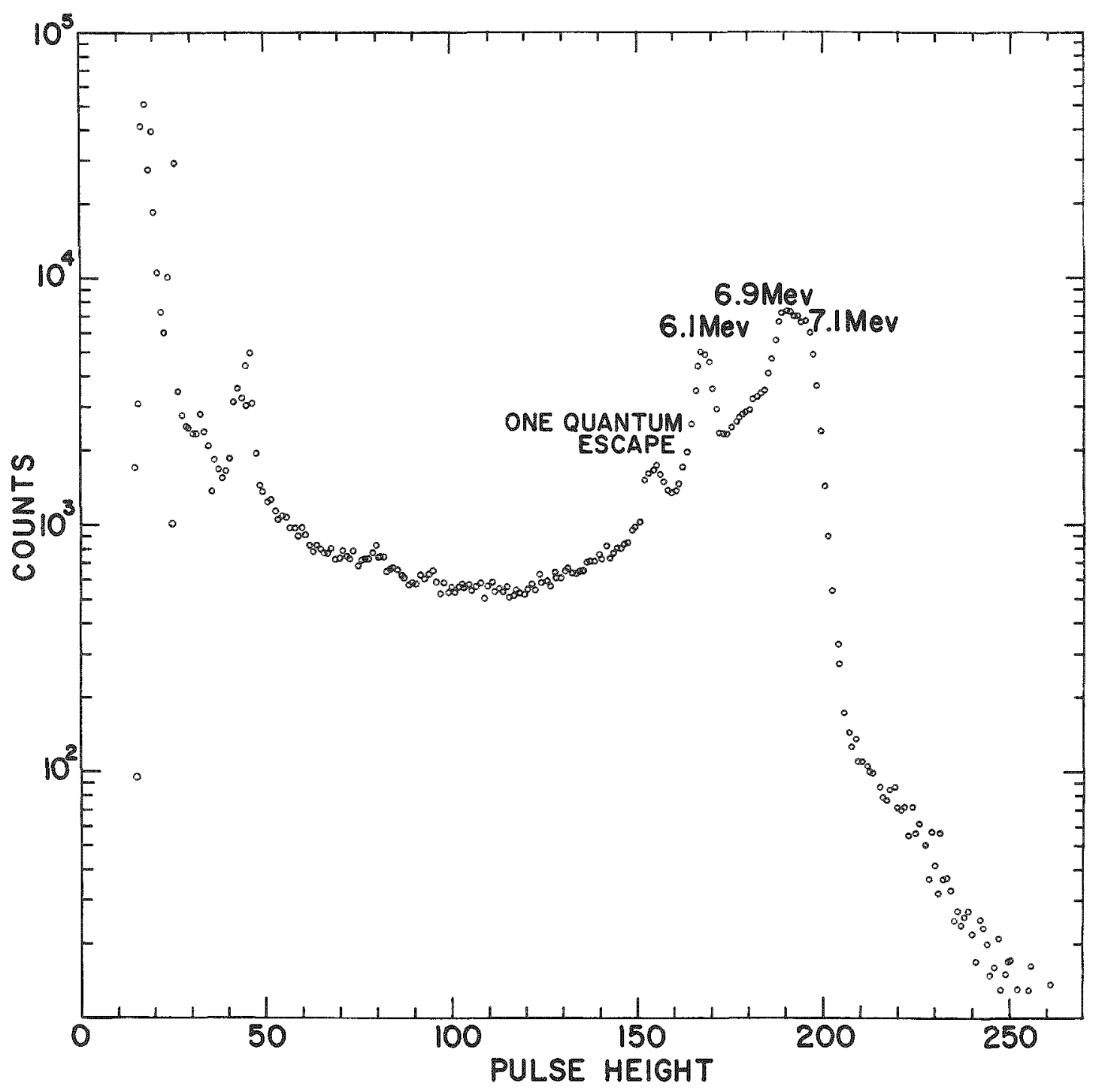

Fig. 7. Gamma rays from $F^{19}(\mathrm{p}, \mathrm{a}) \mathrm{O}$ 15. Energy of bombarding proton is $2.330 \mathrm{Mev}$. 
than its full-energy peak by a calculable amount (about $250 \mathrm{kev}$ ), the four mean positions $j_{k}$ in Eq. (II.9) can be estimated provided the relation between pulse height and energy is known. Further, the intensity, i.e., the product $A_{k} \sigma_{k}$, and width $\sigma_{k}$ of each of these peaks can be calculated from the corresponding parameters of the associated full-energy peaks provided the response of the counter system to an isolated full-energy peak has been determined for gamma rays in this energy region.

set

$$
\text { Let } g_{j} \Delta_{j} \text { be an element of }(j, \bar{j} ; 6, \beta) \text { and consider the }
$$

$$
\left\{g_{j} \Delta_{j}-d_{j} \Delta_{j}: j \leqslant j \leqslant \bar{j}\right\}
$$

where $d_{j}$ is obtained from Eq. (II.9). This set represents the partial spectrum of two full-energy peaks after it has been corrected for room background and for all secondary events. Although the room background is generally negligible in this energy region, we include its effect for completeness. This set can be described in terms of the quantities $F_{j}(\vec{a})$ defined in Eq. (II. 1) provided the second term in Eq. (II.2) is reinterpreted to represent the full-energy peak associated with the line $i=2$ in $\vec{\infty}$. Thus if $\vec{a}$ is the vector which minimizes $Q(\vec{a})$ in Eq. (II. 3), the components of the vectors $\vec{\beta}^{(1)}$ and $\vec{\beta}^{(2)}$ associated with the two full-energy peaks are

$$
\begin{aligned}
& \beta_{1}^{(1)}=a_{1}, \beta_{2}^{(1)}=a_{2}, \beta_{3}^{(1)}=a_{3}, \beta_{4}^{(1)}=0, \\
& \beta_{1}^{(2)}=a_{4}, \beta_{2}^{(2)}=a_{2}-\delta, \beta_{3}^{(2)}=k a_{2}, \beta_{4}^{(2)}=0,
\end{aligned}
$$

and

$$
\beta_{5}^{(1)}+\beta_{5}^{(2)}=a_{5} \approx 0
$$

The energy difference $\delta$ as well as the width ratio $k$ are treated as known in this discussion. However, there is no difficulty in considering $\delta$ as an 
additional parameter, the value of which is to be determined in the same manner as are the values of the components of $\vec{a}$.

In order to extend this analysis to photopeaks of lower energy in the measured spectrum $\bigcup$ it is necessary to subtract from $C$ the contribution from the lines $i=1$ and 2 . In order to carry out this subtraction we assume that a spectrum, say $Q(x)$, of an isolated line has been measured for a gamma-ray energy somewhere in the neighborhood of the energies associated with the photopeaks centered at $\beta_{2}{ }^{(1)}$ and $\beta_{2}{ }^{(2)}$. The proposed procedure is as follows. The spectrum $\sigma^{2}$ is shifted and normalized so that $i t$ is superimposed first on the peak at $1=1$ in $Q$ and then on the peak at $i=2$. The two spectra calculated in this manner are then used to subtract from $\bigodot$ the entire contribution from its two photopeaks from the gamma rays of highest energy. In the example considered here, the gamma rays from $F^{19}(p, a \gamma) 0^{16}$, the $Q^{(x)}$ spectrum is obtained by selecting the proton energy such that the yield of the 6, 1-Mev gamma ray is much more intense than the 6.9- and 7.1-Mev gamma rays. Fig.6 has been used as $Q^{(x)}$ in the analysis of our data on this reaction.

First let us consider the details of this subtraction for the contribution to $@$ from the gamma ray whose full-energy peak is the line $i=1$. Let $Q^{\prime}$ and $Q^{\prime}$ denote the following spectra

$$
Q^{\prime} \equiv y(1, N ; G, B) \text { and } Q^{\prime(x)^{\prime}} \equiv Y_{\left(1, N: e^{(x)}, B\right)}
$$

and let $\vec{a}$ denote the parameter vector corresponding to the single line in

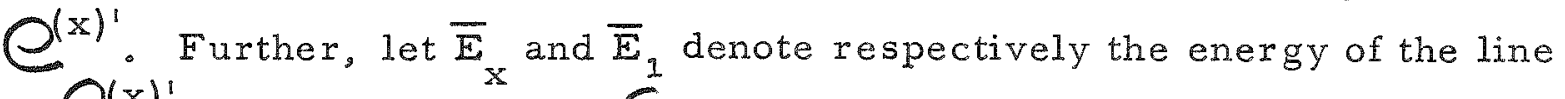
in $Q(x)^{\prime}$ and the $i=1$ line in $E^{\prime}$. Corresponding to an arbitrary energy $E_{I}$ in $\varrho^{\prime}$ is the energy $E_{x}$ in $C^{\prime(x)^{\prime}}$, where

$$
E_{x}=E_{1}-\bar{E}_{1}+\bar{E}_{x} .
$$


If the channel number corresponding to $E_{x}$ in $e^{(x)^{\prime}}$ is $\eta$ and that corresponding to $E_{1}$ in $Q_{1}^{\prime}$ is $j$, then

$$
k \eta=k a_{2}-k^{\prime}\left(\beta_{2}^{(1)}-j\right),
$$

where $k$ and $k^{\prime}$ are the proportionality constants giving the energy in terms of pulse height for $C^{(x)^{\prime}}$ and $C^{\prime}$, respectively. The necessary adjustments on the apparatus can be made to establish this proportionality to a good approximation. If $c_{j}{ }^{\prime} \Delta_{j}$ denotes an element in $\varrho^{\prime}$ and $c_{j}{ }^{(x)} \Delta_{j}{ }^{(x)}$ an element in

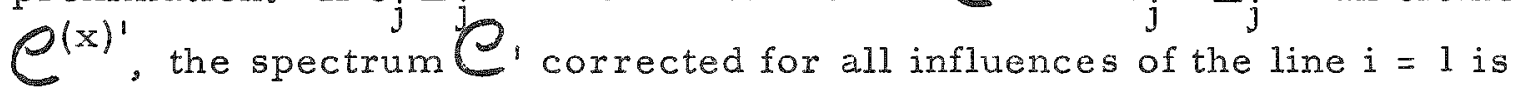

$$
\left\{c_{j} \Delta_{j}-\beta_{1}{ }^{(1)} \beta_{3}{ }^{(I)} c_{\eta}{ }^{(x)} \Delta_{\eta} / a_{1} a_{3}: \eta=a_{2}-k^{\prime}\left(\beta_{2}{ }^{(1)}-j\right) / k ; 1 \leqslant j \leqslant N\right\} .
$$

In order to determine the relation between corresponding channel width $\Delta_{j}$ and $\Delta_{\eta}$, it is necessary that there exist identifiable channel numbers, say $r$ in $e^{(x)^{\prime}}$ and $s$ in $e^{\prime}$, such that they correspond to the same energy. In this case,

$$
k^{1 / k}=r / s
$$

and

$$
\Delta_{\eta}=\left(k^{1} / k\right) \Delta_{j}=(r / s) \Delta_{j}
$$

The set that results from this normalizing and shifting $e^{(x)^{\prime}}$ to match the intensity and mean energy of the $i=1$ line in $Q^{\prime}$ will be denoted by $Q^{(1)}$, i. e.

$$
e^{(1)^{\prime}} \equiv\left\{\beta_{1}{ }^{(1)} \beta_{3}{ }^{(1)} k^{\prime} c{ }_{\eta}^{(x)} \Delta_{j} /\left(a_{1} a_{3}\right): \eta=a_{2}-k^{\prime}\left(\beta_{2}{ }^{(1)}-j\right) / k ; 1 \leqslant j \leqslant N\right\}
$$

By an entirely analogous procedure it is possible to normalize and to shift $e^{(x)^{\prime}}$ so that it is superimposed on the $i=2$ line in $C^{\prime}$. 
Denote this spectrum as $C^{(2)^{\prime}}$. Then in $C^{\prime}$ the influence of the two lines $i=1$ and $i=2$ is simply the sum of corresponding elements in $e^{(1)^{\prime}}$ and $Q^{(2)^{\prime}}$. Thus the set

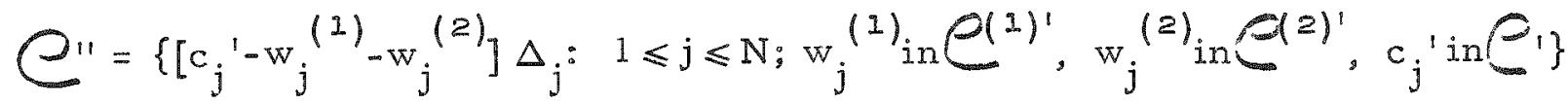
represents the original spectrum corrected for natural background and for all contributions from the gamma rays associated with the lines $i=1$ and $i=2$. The highest energy line $i n Q^{\prime \prime}$ is the line $i=3$ in the original spectrum.

If the photopeaks in 11 are sufficiently well separated and the corresponding component simple spectra can be measured, the set " can be analyzed by means of the procedures outlined in Program I. In those cases in which there is a non-negligible contribution from one-quantumescape processes a term of the form

$$
A \exp \left\{-\left(j-j_{0} / \sigma_{0}\right) 2\right\} \Delta_{j}
$$

should be subtracted from each element in $Q^{\prime \prime}$ in order to correct for these contributions. If any of the conditions necessary for the application of Program I are not satisfied, it is possible to analyze the highest energy lines in $Q^{\prime}$ by repeating the steps given above as Program II with $\bigodot_{\text {" }}$ replacing the set $\left.Y_{(j,}, \bar{j} ; Q y\right)$ wherever it occurs.

The Fitting Procedure: The Newton-Raphson Iteration Method

The vector $\vec{a}$ defined in Eqs. (II.1) and (II.2) describes the response of the spectrometer to an isolated source of monoenergetic gamma rays. The "best estimates" $a_{\ell}$ " of the components of $\vec{a}$ have been defined (thus far as an ad hoc assumption) to be those values for which the quadratic function $Q(\vec{a})$, Eq. (II. 3$)$, attans its minimum value. Since the 
energy of the gamma ray, the intensity of the source, and certain physical properties of the spectrometer system are functions of the components of this vector it is of some interest not only to justify this definition but also to obtain a measure of the accuracy to which such estimates can be calculated for given spectral data.

In Sec.III it is shown that the "best estimates," as defined above, are actually maximum-likelihood estimates and as such they are asymptotically normal and efficient estimates. The variance-covariance matrix associated with their joint probability distribution is given in a form suitable for numerical calculation. Before considering these points, however, let us first outline the numerical procedure that is used to minimize the function $Q(\vec{a})$.

The conditional equations for the evaluation of the vector components that minimize $Q(\vec{a})$ are conveniently written in the form

$$
Q_{\ell}{ }^{\prime}\left(\vec{a}^{*}\right)=0, \quad \ell=1, \ldots, 5,
$$

where, for each component $a_{\ell}$ of $\vec{a}$,

$$
Q_{\ell}{ }^{\prime}(\vec{a}) \equiv \partial Q(\vec{a}) / \partial a_{l}=2 \sum_{j} \Lambda_{j}{ }^{2}\left[F_{j}(\vec{a})-c_{j} \Delta_{j}\right] \partial F_{j} / \partial a_{l} .
$$

The vector $\vec{a}$ specifies the point in five-dimensional parameter space for which $Q(\vec{a})$ attains its minimum value.

The Newton-Raphson method of iteration consists in generating a sequence of successive approximations $\{\vec{a}: \rho=0,1, \ldots\}$ which converges to the limit $\vec{a}^{*}$. The convergence of this sequence depends, of course, on the accuracy of the initial approximation $\vec{a}^{0}$. For the cases of interest here, we have found that a sufficiently accurate initial approximation can be obtained by visual inspection of a measured spectrum. 
Once the components of $\vec{a}^{p}$ have been found for any value of $\rho$, the next term in the sequence is generated by means of a recurrence formula obtained from the expansion

$$
Q_{\ell}\left(\vec{a}^{*}\right)=0=Q_{\ell}^{\prime}\left(\vec{a}^{p}\right)+\sum_{\mathrm{m}} Q_{\ell m}{ }^{\prime \prime}\left(\vec{a}^{p}\right)\left(a_{m} p+1-a_{m}{ }^{p}\right) .
$$

where

$$
\left.Q_{\ell m}{ }^{\prime \prime}(\vec{a})=2 \sum_{j} \Lambda_{j}{ }^{\prime}{ }_{i}\left[F_{j}(\vec{a})-c_{j} \Delta_{j}\right] \frac{\partial 2 F_{j}(\vec{a})}{\partial a_{l} \partial a_{m}}+\frac{\partial F_{j}(\vec{a})}{\partial a_{l}} \frac{\partial F_{j}(\vec{a})}{\partial a_{m}}\right)
$$

If we let $Q^{\prime}(\rho)$ denote the column vector with components $Q_{\ell}^{\prime}\left(\vec{a}^{p}\right)$ and $Q^{\prime \prime}(\rho)$ denote the real symmetric matrix. with components $Q_{\ell m} "(\vec{a} p)$ the solution of the set of equations (II. 14) can be written

$$
\vec{a}^{\rho+1}=\vec{a}^{\rho}-\left[Q^{\prime \prime}(\rho)\right]^{-1} Q^{\prime}(\rho)
$$

where $\left[Q^{\prime \prime}(\rho)\right]^{-1}$ is the inverse of the matrix $Q^{\prime \prime}(\rho)$ and $\vec{a}^{\rho}$ is to be interpreted as a column vector.

The concept of convergence of a sequence of vectors depends on the choice of a vector norm. In the consideration of the conditions sufficient for asymptotic stability of the recurrence relations (II, 16), it is convenient to define the norm of $\vec{a}$ as

$$
\|\vec{a}\|=\max _{\ell}\left|a_{\ell}\right| \text { 。 }
$$

It is then possible to construct a compatible matrix norm in terms of which the conditions for stability of the sequence $\left\{\vec{a}_{0}: \rho=0,1, \ldots, \ldots\right.$ are relatively simple - at least compared with the corresponding conditions which are obtained for the customary definition of vector norm. A complete discussion of this aspect of the iteration procedure is too lengthy to be included in this report. However, it should be mentioned that in the cases 
considered here the repeated application of Eq. (II.16) generates a sequence which converges in the sense that for sufficiently large values of p, the condition

$$
\left\|\vec{a}^{\rho+1}-\vec{a}^{\rho}\right\|<
$$

can be satisfied for any which is larger than the "round-off" error of the computation.

Figure 8 is a flow diagram of the numerical analysis of a complex spectrum. The fitting of the highest-energy photopeak and all isolated photopeaks is actually done three times; once with the background spectrum shifted with respect to the spectrum of interest as indicated by an input datum and twice more with the background curve displaced one channel each way from the initial "guessed" position. The best fit (i。e., the one which gives the lowest value of $Q(\vec{a}))$ is the only one saved. In the subsequent fitting of all lower energy photopeaks the background spec trum is held fixed in the position that yielded the best fit for the highest energy photopeak.

The computer printout consists of the identzfication of the photopeak and the experimental run, the values of $a_{\ell}^{*}$, the value of $Q(\vec{a})$. the error matrix for the joint distribution of the estrmates $a_{\ell}$ as determined by the reciprocal of the matrix $B$ defined below by Eq. (III.95), and the correlation matrix containing the correlation coefficients for the estimates. Also printed for each channel involved in the fitting procedure are the calculated and observed (corrected) counts together with their difference divided by the standard deviation of the observed counts. This latter information is printed also for twelve channels to either side of the interval specified for the fitting routine. 


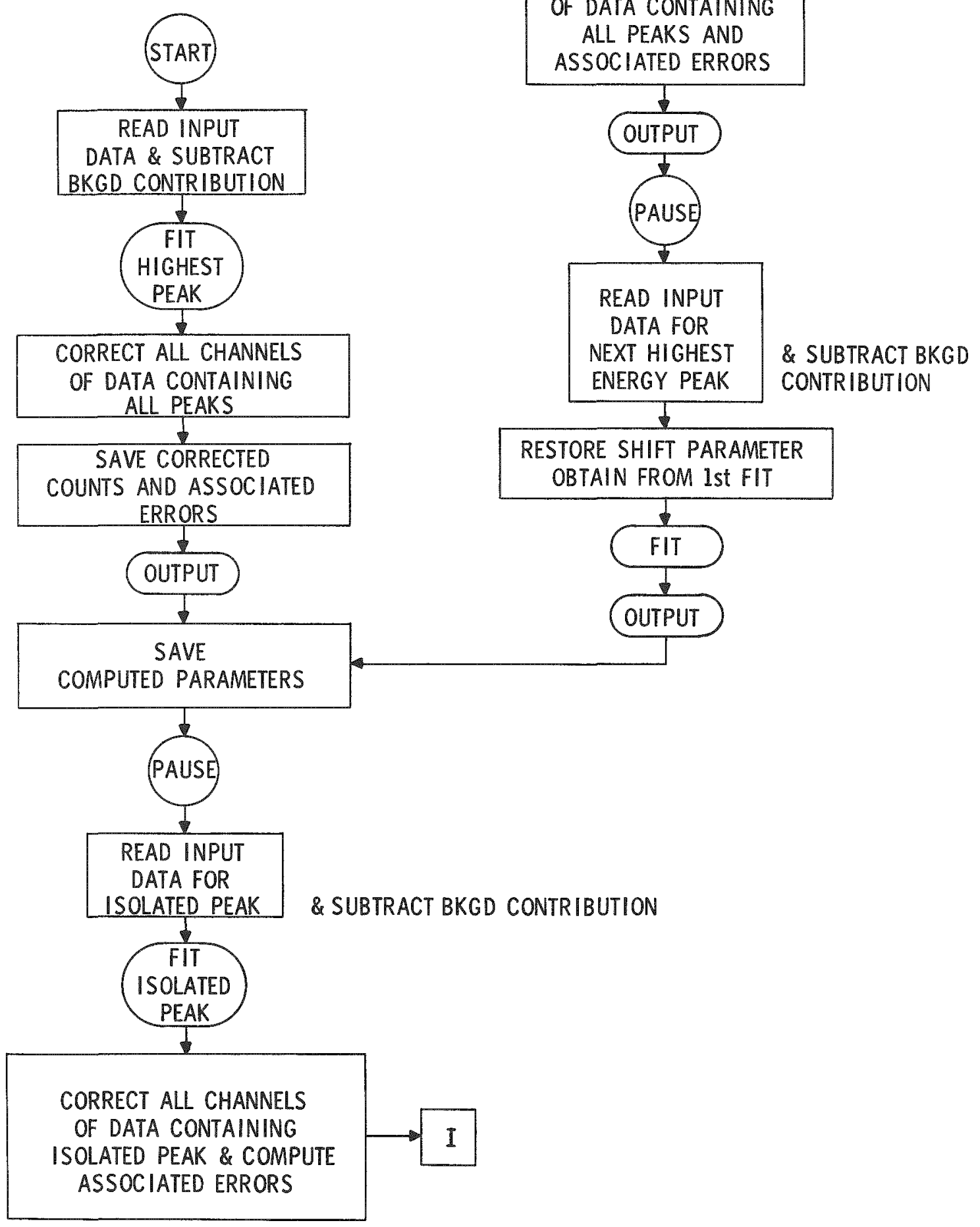

Fig. 8. Flow diagram of the computational procedure. 


\section{THE RESPONSE OF A SPECTROMETER TO MONOENERGETIC GAMMA RAYS}

As is well known, the response of a scintillation spectrometer to a monochromatic gamma ray takes the form of a broad pulse-height distribution. This instrumental distortion of the incoming pulse must be understood if observations made with a crystal spectrometer are to be properly interpreted. In this section we attempt to find an analytic expression which adequately describes this pulse-height distribution. Such an expression will be called the "response function" of the spectrometer system.

As described previously, the spectra obtained by use of a scintillation spectrometer with an anticoincidence annulus are characterized by the suppression, relative to the full energy peak, of those events in which only a part of the energy of the incident gamma ray is converted to the output pulse of the counter. This should make possible a more nearly unique interpretation of the observed response at least in the neighborhood of the full energy peak.

Because of the complex nature of the physical processes that contribute to the formation of the output pulse, the problem of obtaining the actual $x$ esponse function cannot be tackled directly. However, the properties of this distribution function can be summarized in terms of its

moments or other statistical parameters. Several authors ${ }^{3}$ have treated this problem by the use of probability generating functions and have obtained expressions for the first and second moments of the response function. In this formalism, however, the algebra becomes intractable when moments of higher order than the second are considered.

With the help of moment and semi-invariant generating functions we have obtained expressions for the first four semi-invariants (or equivalently for the first four moments) of the response function. From these expressions it is possible to obtain conditions that are necessary in order that the response function approach a Gaussian form and 
also to estimate the extent of deviations of the observed response from normality in practical experimental situations. Such deviations can be of importance in the interpretation of many measurements. For example, the coefficient of skewness of the response function is related directly to the question of the linearity of the energy calibration of the pulse-height spectrum.

In the derivation of expressions for the semi-invariants that characterize the response function it is necessary to repeat some of the considerations contained in previous works on this subject. In particular, the treatment of the formation of the output pulse as a cascade of three events is taken from the review article by Breitenberger. ${ }^{3}$ The reader is referred to this work for a more detailed account of these processes than is given here.

Before we consider the processes by which an output pulse is generated in a spectrometer we briefly review some of the properties of semi-invariants and of moment and semi-invariant generating functions.

\section{Moment and Semi-invariant Generating Functions}

Let $\mathrm{X}$ denote an integer-valued random variable with probability distribution given by

$$
\operatorname{Pr}\{\mathrm{X}=\mathrm{n}\}=\mathrm{p}_{\mathrm{n}} \text { 。 }
$$

The moment generating function $\Omega X^{(\kappa)}$ for this distribution is defined by

$$
\Omega_{\mathrm{X}}(\kappa)=\sum_{\mathrm{n}} \mathrm{p}_{\mathrm{n}} \mathrm{e}^{\mathrm{n} \kappa}
$$

where the summation extends over all integers $n$ for which $p_{n}$ is nonzero. The corresponding semi-invariant generating function $\Psi_{X}(\kappa)$ is defined in terms of $\Omega_{X}(\kappa)$ by the relation

$$
\Psi_{X}(\kappa)=\ln \Omega X^{(\kappa)}
$$


The jth semi-invariant $\lambda_{X}{ }^{(j)}$ of this distribution is obtained from $\Psi_{X}(\kappa)$ by differentiating this function $j$ times with respect to $k$ and evaluating the result for $k$ equal to zero, i.e.

$$
\lambda_{X}^{(j)}=\left.\frac{d^{j} \Psi_{X}^{(k)}}{d \kappa^{j}}\right|_{\kappa=0}=\Psi_{X}^{(j)}(0) .
$$

The first four semi-invariants are related to the central moments of the distribution through the equations

$$
\begin{aligned}
& \lambda_{x^{(2)}}^{(2)}=\langle x\rangle, \\
& \lambda_{x^{(2)}}=\left\langle(x-\langle x\rangle)^{2}\right\rangle, \\
& \lambda_{x^{(3)}}=\left\langle(x-\langle x\rangle)^{3}\right\rangle,
\end{aligned}
$$

and

$$
\lambda_{X}^{(4)}=\left\langle(X-\langle X\rangle)^{4}\right\rangle-3\left[\left\langle(X-\langle X\rangle)^{2}\right\rangle\right]^{2}
$$

where the expectation brackets are defined in the usual manner, viz.,

$$
\left\langle\mathrm{x}^{\kappa}\right\rangle=\sum_{\mathrm{n}} \mathrm{n}^{\kappa} \mathrm{p}_{\mathrm{n}}
$$

The coefficients of skewness $\gamma_{X}^{(2)}$ and excess $\gamma_{X}^{(2)}$ of the distribution are defined by the relations

$$
\gamma_{X}^{(1)}=\lambda_{X}^{(3)} /\left[\lambda_{X}{ }^{(2)}\right]^{3 / 2}
$$

and

$$
\gamma_{X}^{(2)}=\lambda_{X}^{(4)} /\left[\lambda_{X}^{(2)}\right]^{2}
$$

The utility of generating functions arises from the simple manner in which they combine to describe certain compound distributions. We shall have need for the generating functions corresponding to the following possible ways of compounding a distribution from sequences of fundamental distributions. 
a) Alternative events. Suppose an event consists of a sequence $\left\{X_{i}: i=1,2, \ldots.\right\}$ of basic events $X_{i}$ such that at each trial one and only one of the $x_{i}$ occur. If $q_{i}$ is the relative (normalized) frequency of occurrence of the alternative $X_{i}$ in a long sequence of such trials, then the moment generating function corresponding to the composite event $\left\{x_{i}: i=1,2, \ldots ..\right\}$ is given by

$$
\Omega_{X}(\kappa)=\sum_{i} q_{i} \Omega_{X}(\kappa)=\sum_{i} q_{i} \sum_{n} p_{n}^{(i)} e^{n \kappa},
$$

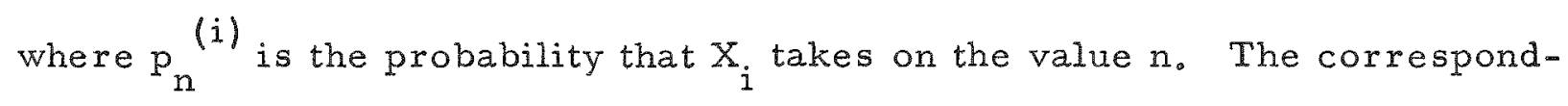
ing semi-invariant generating function is obtained from $\Omega X^{(k)}$ as indicated in Eq. (III. 3).

b) Simultaneous events. Consider the finite sequence $\left\{X_{i}: i=1,2, \ldots . N\right\}$ of independent random variables $X_{i}$, where, for each value of $i$,

$$
\operatorname{Pr}\left\{X_{i}=n\right\}=p_{n}^{(i)}
$$

Let $\mathrm{S}$ denote the random variable defined as

$$
S=\sum_{i=1}^{N} x_{i}
$$

The moment and semi-invariant generating functions for the variable $S$ are

$$
\Omega_{S}(\kappa)=\sum_{i=1}^{N} \Omega_{X_{i}}(K)
$$

and

$$
\Psi_{S}(\kappa)=\sum_{i=1}^{N} \Psi_{X_{i}}(\kappa)
$$


where

$$
\Omega_{X_{i}}(\kappa)=\sum_{n} p_{n}^{(i)} e^{n k}
$$

In the sequence considered here the number $N$ of independent random variables is fixed.

c) Cascade events. Consider next a sequence

$\left\{\mathrm{X}_{\mathrm{i}}: \mathrm{i}=1,2, \ldots ..\right\}$ of mutually independent random variables with the common distribution

$$
\operatorname{Pr}\left\{X_{i}=k\right\}=p_{k} \text { 。 }
$$

Let $S_{N}$ denote the random variable defined by

$$
\mathrm{S}_{\mathrm{N}}=\sum_{\mathrm{k}=1}^{\mathrm{N}} \mathrm{x}_{\mathrm{k}}
$$

where $\mathrm{N}$ is also a random variable, independent of the $\mathrm{X}_{i}$, with the distribution

$$
\operatorname{Pr}\{\mathrm{N}=\mathrm{n}\}=\mathrm{g}_{\mathrm{n}} \text { 。 }
$$

The moment and semi-nnvariant generating functions for the variable $\mathrm{S}_{\mathrm{N}}$ are

$$
\Omega_{S_{N}}(\kappa)=\Omega_{N}\left(\Psi_{X}(\kappa)\right)
$$

and

where

$$
\Psi_{S_{N}}(\kappa)=\Psi_{N}\left(\Psi_{X}(\kappa)\right)
$$

$$
\Omega_{N}(\kappa)=\sum_{n} g_{n} e^{n \kappa}
$$


and

$$
\Psi_{X}(\kappa)=\ln \sum_{k} p_{k} e^{k \kappa}
$$

Let us apply these results to the case of a sequence of simple Bernoulli trials, i.e., consider a sequence of two-valued random variables $\left\{X_{i}: X_{i}=0,1 ; i=1,2, \ldots\right\}$ where, for each value of $i$,

$$
\operatorname{Pr}\left\{X_{i}=1\right\}=P_{i}
$$

and

$$
\operatorname{Pr}\left\{\mathrm{X}_{\dot{\mathrm{I}}}=0\right\}=1-\mathrm{P}_{\mathrm{i}}
$$

The moment generating function for the variable $\mathrm{X}_{i}$ is

$$
\Omega_{X_{i}}(\kappa)=p_{i} e^{\kappa}+\left(1-p_{i}\right)
$$

If the relative frequency of occurrence of the alternative event $X_{i}$ is given as $q_{i}$, the generating function for the sequence is

$$
\Omega_{X}(\kappa)=\sum_{i=1}^{N} q_{i} \Omega_{X_{i}}(\kappa)=P e^{\kappa}+(1-P)
$$

where

$$
P=\sum_{i=1}^{N} q_{i} p_{i}
$$

Thus a sequence of alternative Bernoulli trials is also a Bernoulli trail with probability given by Eq. (III,24). As pointed out by Breitenberger, this seemingly trivial result has an important application in the discussion of the photon transfer efficiency of a scintillation counter.

$$
\text { For a sequence }\left\{X_{i}: X_{i}=0,1 ; i=1,2, \ldots, N\right\} \text { of }
$$
Bernoulli trials with the common distribution 


$$
\operatorname{Pr}\left\{X_{i}=1\right\}=p
$$

the random variable

$$
S=\sum_{i=1}^{N} x_{i}
$$

has the moment generating function

$$
\Omega_{S}(\kappa)=\left[p e^{\kappa}+(1-p)\right]^{N}
$$

If the product $\mathrm{Np}=v$ remains finite as $\mathrm{p}$ approaches zero, the generating function (III.27) approaches the limiting form

$$
\Omega_{S}(\kappa) \rightarrow \exp \left\{\nu\left(\mathrm{e}^{\kappa}-1\right)\right\}
$$

This is the moment generating function for a random variable $S$ that obeys the Poisson distribution

$$
\operatorname{Pr}\{s=s\}=\nu s e^{-\nu / s !}
$$

where $\nu$ is the expected value of $s$. The semi-invariant generating function for the Poisson distribution is

$$
\Psi_{S}(\kappa)=\nu\left(e^{K}-1\right)
$$

\section{Application to the Scintillation Counter}

The mechanism by which an output pulse is generated in a scintillation counter system can be discussed as a cascade of three processes: 
i) The absorption of energy by the phosphor and the subsequent emission of photons.

i1) The collection of these quanta at the cathode of the photomultiplier tube and the resulting ejection of photoelectrons by the cathode.

1ii) The subsequent multiplication of these photoelectrons by successive dynode stages in the photomultiplier tube

Given the generating function corresponding to the probdbility distribution of each of these processes, the above methods of compounding sequences of events can be used to obtain the generating function for the distribution of the output pulse for an incident monoenergetic gamma ray. Each of these cascade events will be considered separately in an effort to obtain reasonable approximations to their generating functions.

\section{a) Generation of light quanta in the scintillator. One of} three primary processes may take place when a gamma ray photon traverses a NaI crystal. These are photoelectric absorption; Compton scattering, or pair production. In each of these processes energy is transmitted to electrons in the crystal and the se energetic electrons interact with the atoms of the crystal. Ultimately a fraction of this excitation energy is emitted as light in a wave length band which the photomultiplier can detect and amplify. Here it will be assumed that the entire energy of the incident gamma ray is transmitted to the crystal in each of these primary processes. Such an assumption is strictly valid in the case of photoelectric absorption provided that the energetic electrons thus produced, do not escape from the crystal nor produce bremsstrahlung which escapes from the crystal. The assumption is applicable also to the case of pair production if neither particle of the pair escapes nor produces bremsstrahlung which escapes the crystal; furthermore, after the annihilation of the positron both of the annihilation quanta must be stopped within the crystal. The assumption of complete energy transfer in the case 
of Compton scattering is equivalent to the "thick case" approximation discussed by Seitz and Mueller. If ${ }^{4}$ represents the efficiency with which a luminescent crystal converts the excitation energy it receives into light quanta, the average number $\bar{x}$ of light quanta produced by an incident gamma ray of energy $E$ is given by

$$
\bar{x}=\xi E
$$

For a thallium-activated sodium iodide crystal, $\xi$ is of the order of $104 / \mathrm{Mev}$ and is assumed to be independent of the energy of the incident gamma ray.

It is convenient at this point to drop the convention of using capital letters to denote random variables. Let $\mathrm{x}$ be the random variable whose value is the number of light quanta produced in a NaI crystal by a gamma ray of energy $\mathbf{E}$.

The nature of the luminescence process itself is not completely understood and it is difficult to justify any particular distribution for the variable $x$. However, the general features of this process satisfy the conditions for a Poisson distribution as may be seen by considering the production of $\mathrm{x}$ quanta as a sequence of simultaneous Bernoulli trials each with the same probability for the production of a light quantum. It is therefore assumed that the semi-invariant generating function for the $r$ andom variable $x$ is

$$
\Psi_{x}(\kappa)=\bar{x}\left(e^{\kappa}-1\right)
$$

b) Collection of light quanta. Only a fraction t of the light quanta produced in the crystal contributes to the initiation of the secondary emission avalanches at the first dynode of the photomultiplier. Specifically, a successful photon transfer is a cascade of four Bernoulli-type events: 
(1) The light quanta find their way (with probability e) from the phos phor into the optical system which guides them toward the cathode of the photomultiplier. (2) Some of the quanta in the optical system arrive (with probability $f$ ) at the cathode. A fraction $(1-f$ ) of these quanta are lost by absorption and reflection en route from the phosphor to the cathode. (3) A light quantum striking the cathode releases (with probability p) a photoelectron. (4) Photoelectrons from the cathode initiate (with probability c) secondary emission avalanches at the first dynode. Since each step in this sequence is a Bernoulli event, the entire process of photon transfex is also a Bernoulli event with probability $t$ for successful transfer of the photon from the phosphor to the first dynode of the photomultiplier tube. Furthermore, by Eq. (III.23), this transfer efficiency is just the product of the individual probabilities for each step in the sequence, i.e.,

$$
t=\operatorname{efpc}
$$

Successive scintillations, however, never occur at exactly the same point inside the phosphor and photons produced at different points have different escape probabilities. This implies that the probability $t$ will vary from one scintillation to the next. Let $\left\{t_{i}: i=1,2, \ldots ..\right\}$ denote the sequence of possible transfer probabilities for each photon in a given scintillation and suppose that a given value $t_{i}$ occurs with relative frequency $q_{i}$ in a series of many scintillations. The transfer efficiencies thus form a sequence of alternative events such that for each photon one and only one of the $t_{i}$ occur. The moment generating functions for these alternatives are

$$
\Omega_{t_{i}}(\kappa)=t_{i} e^{\kappa}+\left(1-t_{i}\right)
$$

for all $t_{i}$. 
c) The cascade of secondary electrons. The photoelectron that arrives at the first dynode produces several secondary electrons. In a multistage multiplier tube, these secondaries in turn become primaries with respect to another dynode and on impact produce several secondaries each. Because of the random nature of the process at each step the cumulative effect on the output pulse after a given number of multiplication stages can also be treated as a cascade of events. Such a calculation has been carried out by Woodward. ${ }^{5}$ However, the only property which we shall assume for this distribution is the existence of finite semi-invariants of all orders. In the discussion that follows $\Psi_{m}(\kappa)$ will denote the semiinvariant generating function for the tube gain variable $m$ and $\lambda_{m}(j)$ the corresponding jth semi-invariant.

\section{Semi-invariants of the Pulse-height Distribution}

If an incident gamma ray of energy E initiates the emission of $x$ light quanta from the phosphor, the number $S_{x}$ of photoelectrons which arrive at the first dynode is given by

$$
S_{x}=\sum_{k=1}^{x} t_{k} q_{k} .
$$

The generating functions for the distributions of the random variables $x$ and $t_{k}$ are given respectively by Eqs. (III.32) and (III.34). If $m_{i}$ denotes the tube gain for the ith such photoelectron the resulting pulse height $\mathrm{q}$ is obtained as the sum of the $S_{x}$ values $m_{i}, i_{0} e_{0}$,

$$
q=\sum_{i=1}^{s} m_{i} .
$$

The $m_{i}$ are assumed to have a common distribution defined by the semiinvariant generating function $\Psi_{m}(\kappa)$. Reference to Eq. (III. 17) reveals that the moment generating function $\Omega_{q}(\kappa)$ for the pulse height $q$ can be 
expressed in the form

$$
\Omega_{\mathrm{q}}(\kappa)=\Omega_{\mathrm{S}}\left(\Psi_{\mathrm{m}}(\kappa)\right)
$$

According to Eqs。(III, 8) and (III, 17) and the discussion preceding Eq. (III. 17), the moment generating function for $\mathrm{S}_{\mathrm{X}}$ is

$$
\Omega_{S}(\kappa)=\sum_{i} q_{i} \Omega_{x}\left(\Psi_{t_{i}}(\kappa)\right),
$$

where $q_{i}$ is the frequency with which the transfer probability $t_{i}$ occurs. The $\mathrm{q}_{\mathrm{i}}$ are normalized such that

$$
\sum_{i} q_{i}=1
$$

Combining these results leads to the expression

$$
\Omega_{q}(\kappa)=\sum_{i} q_{i} \Omega_{x}\left(\Psi_{t_{i}}\left(\Psi_{m}(\kappa)\right)\right)=\sum_{i} q_{i} \exp \left\{\bar{x}_{i}\left(e^{\Psi}(\kappa)-1\right)\right\},
$$

for the moment generating function of the random variable q. Expressions (III.32) and (III.34) have been substituted for the generating functions $\Omega_{\mathrm{x}}$ and $\Psi_{t_{i}}$ in obtaining this last relation.

The first four semi-invariants of the pulse height distribution are obtained from Eq. (III,40) as indicated in Eqs。(III,3) and (III,4)。 The result is

$$
\begin{aligned}
& \lambda_{\mathrm{q}}{ }^{(1)}=\overline{\mathrm{x}} \lambda_{\mathrm{t}}{ }^{(1)} \lambda_{\mathrm{m}}{ }^{(1)} \\
& \lambda_{q}^{(2)}=\bar{x} \lambda_{t}^{(1)}\left[\left(\lambda_{m}^{(1)}\right)^{2}+\lambda_{m}^{(2)}\right]+\bar{x}^{2}\left(\lambda_{m}{ }^{(1)}\right)^{2} \lambda_{t}^{(2)} \\
& \lambda_{\mathrm{q}}^{\left({ }^{(3)}\right.}=\bar{x} \lambda_{\mathrm{t}}{ }^{(1)}\left[\left(\lambda_{\mathrm{m}}{ }^{(2)}\right)^{3}+3 \lambda_{\mathrm{m}}{ }^{(2)} \lambda_{\mathrm{m}}{ }^{(2)}+\lambda_{\mathrm{m}}{ }^{(3)}\right] \\
& +3 \bar{x}^{2} \lambda_{t}{ }^{(2)}\left[\left(\lambda_{m}{ }^{(2)}\right)^{3}+\lambda_{m}{ }^{(1)} \lambda_{m}{ }^{(2)}\right]+\bar{x}^{3}\left(\lambda_{m}{ }^{(1)}\right)^{3} \lambda_{t}^{(3)}, \quad \text { (III。4lc) }
\end{aligned}
$$


and

$$
\begin{aligned}
& \lambda_{\mathrm{q}}{ }^{(4)}=\bar{x} \lambda_{\mathrm{t}}{ }^{(1)}\left[\left(\lambda_{\mathrm{m}}{ }^{(1)}\right)^{4}+6\left(\lambda_{\mathrm{m}}{ }^{(1)}\right)^{2} \lambda_{\mathrm{m}}{ }^{(2)}+3\left(\lambda_{\mathrm{m}}{ }^{(2)}\right)^{2}+4 \lambda_{\mathrm{m}}{ }^{(1)} \lambda_{\mathrm{m}}{ }^{(3)}+\lambda_{\mathrm{m}}{ }^{(4)}\right] \\
& +\bar{x}^{2} \lambda_{t}^{(2)}\left[14 \lambda_{m}{ }^{(1)} \lambda_{m}{ }^{(3)}+18\left(\lambda_{m}{ }^{(1)}\right)^{2} \lambda_{m}^{(2)}+3\left(\lambda_{m}{ }^{(2)}\right)^{2}+7\left(\lambda_{m}{ }^{(1)}\right)^{4}\right] \\
& +6 \bar{x}^{3} \lambda_{t}^{(3)}\left[\left(\lambda_{m}^{(1)}\right)^{4}+\left(\lambda_{m}{ }^{(1)}\right)^{2} \lambda_{m}^{(2)}\right]+\bar{x}^{4} \lambda_{t}^{(4)}\left(\lambda_{m}^{(1)}\right)^{4}
\end{aligned}
$$

where $\lambda_{q}{ }^{(j)}, \lambda_{m}{ }^{(j)}$, and $\lambda_{t}{ }^{(j)}$ denote respectively the semi-invariants of the distributions of the random variables $q, m$, and $t$, The semi-invariant $\lambda_{t}{ }^{(j)}$ is defined by Eq. (III.5) in terms of the moments $\left\langle t^{j}\right\rangle$ where

$$
\left\langle t^{j}\right\rangle=\sum_{i} q_{i} t_{i}^{j}
$$

The relative variance $\mathbb{1}_{\mathrm{q}}$ of the pulse-height distribution is defined as the ratio $\lambda_{q}{ }^{(2)} /\left(\lambda_{q}(1)\right)^{2} \cdot$ From Eqs. (III.41a) and (III. 41b), it follows that

$\mathcal{L}_{\mathrm{q}}=\lambda_{t}^{(2)} /\left(\lambda_{\mathrm{t}}^{(1)}\right)^{2}+\left[1+\lambda_{\mathrm{m}}^{(2)} /\left(\lambda_{\mathrm{m}}^{(1)}\right)^{2}\right] /\left(\bar{x} \lambda_{t}^{(1)}\right)=\mathcal{L}_{t}+\left(1+\mathcal{L}_{\mathrm{m}}\right) /\left(\bar{x} \lambda_{t}^{(1)}\right)$,

where $\mathbb{N}_{t}$ and $\mathbb{1}_{\mathrm{m}}$ are the relative variances of the distributions of $t$ and (III.43) $m$, respectively. The coefficients of skewness and excess are defined in terms of the semi-invariants by Eq. (III.7). For the pulse-height distribution, these coefficients can be obtained from Eqs. (III.4Ia) through (III.41d). They are

$\left.\gamma_{q}^{(1)}=\mathcal{L}_{q}^{-3 / 2} \sum_{\left[\frac{1+3 \mathcal{D}_{m}+\Lambda_{m}}{(3)}\right.}^{\left(\bar{x} \lambda_{t}^{(1)}\right)^{2}}+\frac{3 \mathcal{L}_{t}\left(1+\mathcal{L}_{m}\right)}{\bar{x} \lambda_{t}^{(1)}}+\Lambda_{t}^{(3)}\right]^{\prime}$ 
and

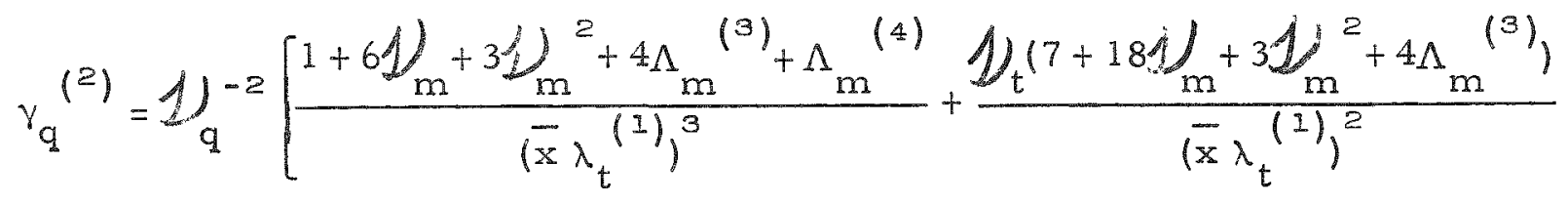

$$
\begin{aligned}
& \left.+\frac{6 \lambda_{t}^{(3)}(1+1)}{\left(\bar{x} \lambda_{t}^{(1)}\right)}+\Lambda_{t}^{(4)}\right]
\end{aligned}
$$

where

$$
\left.\Lambda_{m}^{(j)}=\lambda_{m}^{(j)} / \lambda_{m}^{(I)}\right)^{j}
$$

and.

$$
\Lambda_{t}^{(j)}=\lambda_{t}^{(j)} /\left(\lambda_{t}^{(I)}\right)^{j}
$$

In principle, at least, each of these statistics can be measured as a function of the energy $E=\zeta^{-1} \bar{x}$ of the incident gamma ray. In Sec. IV are given the results of such measurements of $\mathbb{L}_{q}$ and $\gamma_{q}{ }^{(1)}$.

\section{The Response Function}

The standardized ${ }^{\text {thh }}$ semi-invariant $\lambda_{q}^{(j)} /\left(\lambda_{q}{ }^{(2)}\right)^{j / 2}$ for the $\mathrm{q}$ distribution as defined by its generating function (III.40) behaves for large values of $\bar{x}$ as

$$
\lambda_{q}^{(j)} /\left(\lambda_{q}{ }^{(2)}\right)^{j / 2}-\Lambda_{t}^{(j)}+\ldots
$$

where the remaining terms are of the first and higher order in $1 / \bar{x}$. If the ratio $\Lambda_{t}(j)$ is zero for $j \geqslant 3$, then as $\bar{x}$ approaches infinity each semiinvariant of order greater than two vanishes for the q distribution. This condition, if it is satisfied, uniquely determines the asymptotic form of 
the pulsemeight distribution to be Gaussian. In this case the asymptotic frequency function for the random variable $q$ becomes

$$
f(q) d q=\frac{1}{\sigma \sqrt{2 \pi}} \exp \left[-\frac{1}{2}\left(\frac{q-q_{0}}{\sigma}\right)^{2}\right] d q \text {. }
$$

where

$$
q_{0}=\bar{x} \lambda_{m}^{(1)} \lambda_{t}^{(1)}
$$

and

$$
\sigma^{2}=q_{0} 21 L_{q}
$$

Note that the asymptotic values of the coefficients of skewness and excess are zero provided the third-and fourth-order semi-invariants of the $t$ distribution vanish.

Deviations of an observed response function from this simple asymptotic form depend not only on the assumptions explicitly mentioned in this derivation but also on many factors which have been ignored in the discussion. For example, the electronic equipment was not mentioned although it is always a source of small parasitic fluctuations. Many of these "secondary" effects are discussed in reference (3). It is quite obvious that, for any given experimental setup, the nature and extent of any differences between the observed response function and the simple form of Eq. (III.47) must be obtained experimentally.

One direct method of obtaining such a measurement is to compare a measured full-energy pulse-height distribution with the Edgeworth distribution, viz.,

$$
\phi(q)=f(q)-\frac{\gamma_{q}^{(1)}}{3 !} f^{(3)}(q)+\frac{\gamma_{q}^{(2)}}{4 !} f^{(4)}(q)+\ldots
$$


where $f(q)$ is the Gaussian function (III.47) and

$$
f^{(v)}(q)=(-1)^{v} H_{v}(q) f(q)
$$

Here $\mathrm{H}_{v}(\mathrm{q})$ is the Hermite polynomial of order $v$.

The results obtained in preliminary measurements of this kind are discussed in Sec.IV. At this point it suffices to say that Eq. (III.47) satisfactorily describes the observed pulse-height response for values of $q$ that satisfy the inequality

$$
\left|q-q_{0}\right| \leq 2.5 \sigma
$$

These limits correspond to roughly $97 \%$ of the total number of counts in any full-energy peak. (The word "satisfactorily" in the above context means that the measured deviations from Eq. (III.47) were such that they could be attributed to expected statistical fluctuations in counting. This will be discussed in Sec. IV.)

\section{Maximum-Likelihood Estimates}

The present problem of obtaining estimates of the values of the components of the vector $\vec{a}$ differs from the usual problems in statistical estimation in that the total number of events in the spectrum, i.e., the value of $\sqrt{\pi} a_{1} a_{3}$, is not specified in advance. As a practical matter, the entire observed spectrum $\left\{c_{j} \Delta_{j}^{a} j=1, \ldots, N\right\}$ must be truncated (somewhat arbitrarily) so that only the data included in the subset

$$
C=\left\{c_{j} \Delta_{j} \equiv c_{j}: \underline{j} \leqslant j \leqslant \bar{j}\right\}
$$

is available for the estimation of the values of $a_{\ell}$. In this subsection we develop a method for obtaining maximum-likelihood estimates from such truncated spectra. Also the limiting form of the joint probability distribution of these estimates is discussed. 
For the present we treat the background subtractions as nonrandom processes. The modifications necessary for the inclusion of these corrections as statistical processes are given in the subsections which immediately follow this subsection.

First we consider the probability of observing the data represented by the set $($ Let $m$ be the total number of counts actually observed in $\rho_{\text {i. e. }}$

$$
m=\sum_{j \in Q} c_{j}
$$

Given that the total number of counts in the complete (nontruncated) spectrum is $N$, the probability of obtaining 0 as a measured result is

$$
\operatorname{Pr}\{\operatorname{C} \mid N\}=\frac{N !}{(N-m) !}(1-p)^{N-m} \prod_{j \in P} \frac{p_{j}^{c_{j}}}{c_{j}^{j}},
$$

where $\mathrm{p}_{j}$, the probability of a single count occurring in the jth channel, is

$$
p_{j}=p_{j}(\vec{a})=\frac{1}{a_{3} \sqrt{\pi}} \int_{j-\frac{1}{2}}^{j+\frac{1}{2}} d x \exp \left[\frac{-\left(x-a_{z}\right) \geq}{a_{3} z^{2}}\right],
$$

and

$$
p=\sum_{j \in C^{p}} p_{j}
$$

[In Eq. (III.55) and throughout this section we use the notation $f(\vec{a})$ to indicate a function of one or more of the components of the vector $\vec{a}$. In many instances where there is little chance of confusion, the dependence of $f$ on $\vec{a}$ will not be written explicitly as, e.g., in Eq. (III.56).] In the present problem $\mathrm{N}$ is itself a random variable whose distribution is assumed to be that given by the Poisson law

$$
\operatorname{Pr}\{N\}=\mathbb{M}^{N} e^{-M} / N !
$$


where $M=\sqrt{\pi} a_{1} a_{3}$ is the expected total number of counts in the nontruncated spectrum. The probability of observing the truncated spectrum with no a priori knowledge of the value of $N$, other than the obvious condition that

$$
N \geqslant m \text {, }
$$

is given by a combination of probability distributions, namely,

$$
\operatorname{Pr}\{e\}=\sum_{N=m}^{\infty} \operatorname{Pr}\{e \mid N\} \operatorname{Pr}\{N\}=e^{-M p} \operatorname{T/}_{j \in C}\left(\operatorname{Mp}_{j}\right)^{\mathrm{c}} / \mathrm{c}_{j} !
$$

The mean value, variance, and covariance of the variables $c_{j}$ in this distribution are respectively

$$
\bar{c}_{j}=M_{j^{\prime}} \quad \operatorname{var}\left(c_{j}\right)=M_{p^{\prime}} \quad \text { and } \quad \operatorname{cov}\left(c_{j^{\prime}}, c_{j^{\prime}}\right)=0
$$

This is most easily shown by observing that the moment-generating function for the probability distribution $\operatorname{Pr}\{C\}$ is

$$
\mathscr{Y}\left(s_{j}: j \in Q\right)=\exp \left\{-M\left(p-\sum_{j \in \ell} p_{j} s_{j}\right)\right\}
$$

The maximum-likelihood estimates $a_{\ell}^{* k}$ for the values of $a_{\ell}$ are defined by the condition that the probability $\operatorname{Pr}\{\Omega$ of obtaining the

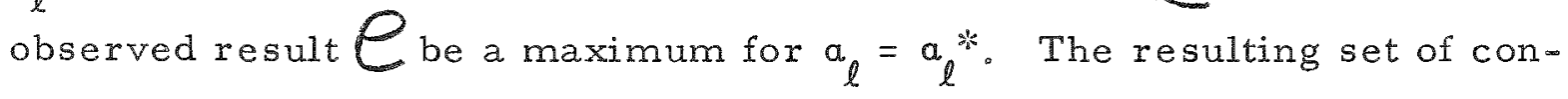
ditions for the determination of the estimates is then

$$
\left.\sum_{j \in \ell}^{l} \mid \frac{c_{j}}{F_{j}(\vec{a} *)}-1\right] \quad \frac{\partial F_{j}\left(\vec{a}_{*}^{*}\right)}{\partial a_{\ell}}=0, \quad l=1, \ldots, 5
$$

In the derivation of Eq. (III.61) we have used the relation

$$
F_{j}(\vec{a})=M(\vec{a}) p_{j}(\vec{a})
$$

which follows from the definition, Eq. (II. 1), of $F_{j}(\vec{a})$. 
From the definition of the weights $\Lambda_{j}{ }^{2}$ in Eq. (III.3) coupled with Eq. (IV.13) it follows that

$$
\Lambda_{j}^{2} \approx<1 / \operatorname{var}\left(c_{j}\right) \approx 1 / F_{j}\left(\vec{a}^{*}\right)
$$

With this result, the conditional equations (III.61) for the maximumlikelihood estimates are seen to be completely equivalent to the conditional equations (II. 10) for the minimization of $Q(\vec{a})$.

Next we consider some properties of the joint probability distribution of the estimates $a_{\ell} \%$. The meaning of a probability distribution for these components is the following. From a sequence of similar measurements select the subsequence which resulted in the same spectrum Q. Then the distribution of the estimates $a_{\ell}$ over this subsequence is the joint distribution in which we are interested. The necessity of this definition may be understood by a consideration of the meaning of the averaging process carried out below in Eq. (III.75). Actually, we must content our selves with a discussion of this distribution in the limit in which the number of independent measurements in the above subsequence approaches infinity。

Let

$$
\mathcal{X}(\vec{a})=\ln \operatorname{Pr}\{Q\}=\sum_{j \in C} \ln L_{j}\left(c_{j} \mid \vec{a}\right) .
$$

where, from Eq. (III. 58), it is seen that $L_{j}\left(c_{j} \mid \vec{a}\right)$ is the Poisson distribution for observing $c_{j}$ events in channel $j$ when the average number of such events is $M p_{j}=F_{j}$, i.e,

$$
L_{j}\left(c_{j} \mid \vec{a}\right)=e^{-F_{j}(\vec{a})}\left[F_{j}(\vec{a})\right]^{c_{j}} / c_{j} !
$$


The notation $L_{j}\left(c_{j} \mid \vec{a}\right)$ is used to denote the conditional distribution of $c_{j}$ when the values of the components of $\vec{a}$ are given. Consider the Taylor expansion

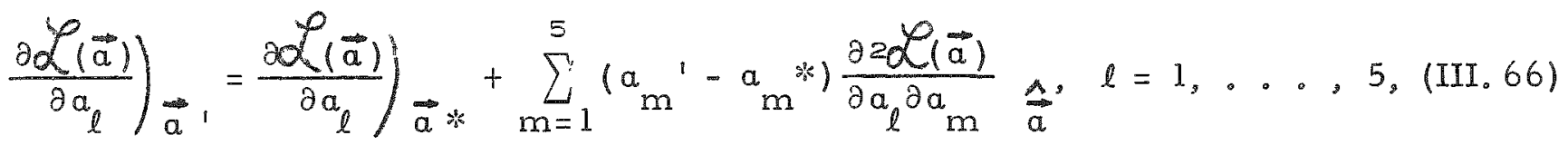
where the $a_{l}$ ' are the "true values" of the components of $\vec{a}$, the $a_{l} *$ are the corresponding maximum-likelihood estimates, and the vector $\hat{a}$ is defined such that $\hat{a}_{l}$ exists in the interval $\left(a_{l}{ }^{*}, a_{l}{ }^{\prime}\right)$ for all values of $l_{0}$. By definition of maximum-likelihood estimates the first term on the righthand side of Eq. (III.66) vanishes and, if $a_{\ell}$ * converges stochastically to

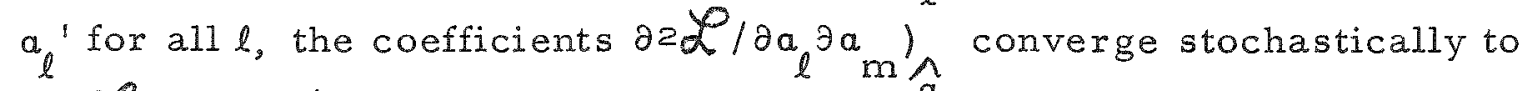
$\left\langle\partial 2 \mathcal{L} / \partial a_{\ell} \partial a_{m}\right\rangle$; Here the subscript on " the "expectation brackets" denotes that the averaging process is to be carried out under the hypothesis that $a_{\ell}=a_{\ell}{ }^{\prime}$ for all $\ell$. The joint distribution of the $a_{\ell}{ }^{*}$ is not affected by the substitution of this expectation value so that in the limit Eqs. (III. 66) become

$\left.\frac{\partial \mathcal{Q}(\vec{a})}{\partial a_{l}}\right)_{\vec{a}}=\sum_{m=1}^{5}\left(a_{m} \cdot-a_{m}\right)^{*} B_{l n}, \quad l=1, \ldots, 5$,

where

$$
B_{\ell n}=\left\langle\partial 2 \mathcal{L}(\vec{a}) / \partial a_{\ell} \partial a_{m}\right\rangle_{\vec{a}}, \quad \ell, m=1, \ldots, 5 .
$$

Carrying out the averaging process leads to

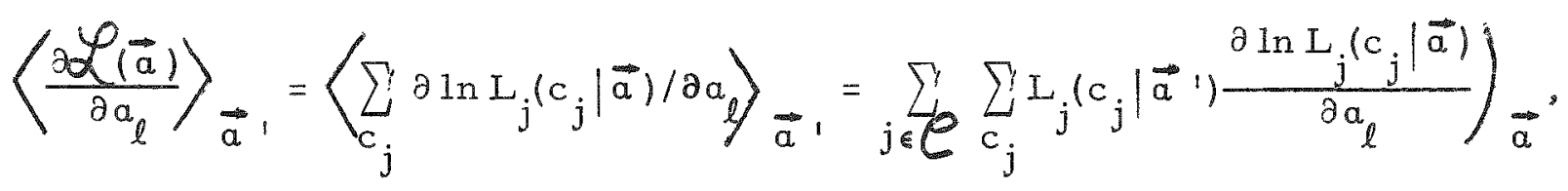

$$
\begin{aligned}
& =\sum_{j \in \ell} \frac{\partial}{\partial a_{\ell}}\left[\sum_{c_{j}} L_{j}\left(c_{j} \mid \vec{a}\right)\right]_{\vec{a}_{1}}=0,
\end{aligned}
$$


since

$$
\sum_{c_{j}} L_{j}\left(c_{j}|\vec{a}|\right)=1
$$

and

$\left.B_{l m}=\sum_{j \in j} \sum_{c_{j}} L_{j}\left(c_{j} \mid \vec{a}^{\prime}\right) \frac{\partial z_{w}}{\partial a_{\ell} \partial a_{m}}\right)_{\vec{a}^{\prime}}=-\sum_{j \in=} \sum_{c_{j}}\left[\frac{1}{L_{j}} \frac{\partial L_{j}}{\partial a_{\ell}} \frac{\partial L_{j}}{\partial a_{m}}\right\rfloor_{\vec{a}^{\prime}}$,

where

$$
\frac{\partial L_{j}}{\partial a_{\ell}}=\left[\frac{c_{j}}{F_{j}}-1\right] \frac{\partial F_{j}}{\partial a_{\ell}} L_{j} .
$$

From the definition of expectation value and Eq. (III.59) we obtain

$$
\sum_{c_{j}} L_{j}\left(c_{j} \mid \vec{a}^{\prime}\right)\left[c_{j}-F_{j}\left(\vec{a}^{\prime}\right)\right]^{2}=\operatorname{var}\left(c_{j}\right)=F_{j}\left(\vec{a}^{\prime}\right)
$$

The substitution of Eqs. (III.72) and (III.73) into Eq. (III.71) gives

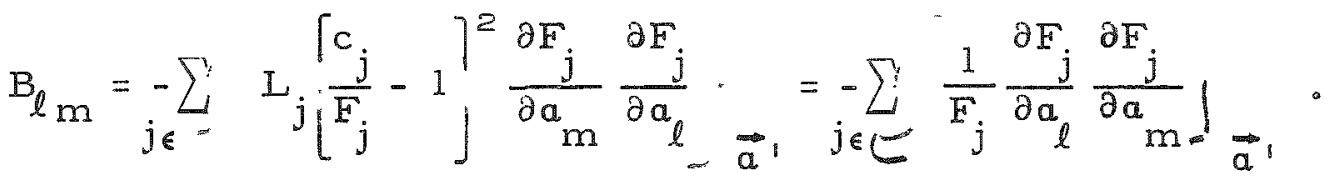

Let $\left(B^{-1}\right)_{\ell m}$ denote a component of the reciprocal of the symmetric matrix whose components are defined by Eq. (III. 74). The solution of the set of equations (III.67) for $a_{m}^{\prime}-a_{m} *$ is then

$\left.a_{m}^{\prime}-a_{m} *=\sum_{\ell=1}^{5}\left(B^{-1}\right)_{m \ell} \partial \mathcal{C}^{\prime}(\vec{a}) / \partial a_{\ell}\right)_{\vec{a}^{\prime}}, \quad m=1, \ldots, 5$.

The components of the error matrix which specify the joint asymptotic distribution of the $a_{m} *$ are obtained by carrying out the averaging process over a subset of measurements yielding the spectrum $\bigcirc$ as discussed above. The result is 
$\left\langle\left(a_{m}{ }^{\prime}-a_{m}^{*}\right)\left(a_{r}{ }^{\prime}-a_{r}^{*}\right)\right\rangle=\sum_{\ell, t=1}^{5}\left(B^{-1}\right)_{m \ell}\left(B^{-1}\right)_{r t}\left\langle\frac{\partial \mathcal{L}}{\partial a_{\ell}} \frac{\partial \mathscr{L}}{\partial a_{t}}\right\rangle_{\vec{a}^{\prime}}=-\left(B^{-1}\right)_{m r}$

since

$$
\left\langle\frac{\partial \mathcal{L}}{\partial a_{\ell}} \frac{\partial \mathcal{L}}{\partial a_{t}}\right\rangle_{a_{1}}=\sum_{j \in \varrho_{j}} \sum_{c_{j}}\left\{\frac{1}{L_{j}} \frac{\partial L_{j}}{\partial a_{l}} \frac{\partial L_{j}}{\partial a_{t}}\right\}_{a^{\prime}}=-B_{\ell t} .
$$

\section{Probability Distribution of the Corrected Counts}

In this subsection we consider the probability distribution of the counts $c_{j} \Delta_{j}$ in any of the spectra corrected for "background" by the methods discussed in Sec. II. Knowledge of this distribution is necessary in order that the se background subtractions can be included as random processes which can contribute unequal weights to the data points $c_{j} \Delta_{j}$ in the estimation of the values of the corresponding response parameters $a_{\ell}$.

Let us as sume that the counts $a_{j}$ actually measured in the jth channel obey a Poisson distribution, the expected value of $a_{j}$ being denoted by $\bar{a}_{j}$. Further, let us suppose that the associated background counts $b_{j}$ for this channel also obey a Poisson distribution with expected value $\bar{b}_{j}$. Each of the subtraction techniques discussed in Sec. II can be written in the form

$$
c_{j}=a_{j}-\theta_{j} b_{j},
$$

where $\theta_{j}$ is a numerical constant for any channel and the $c_{j}$ are elements of the corrected spectrum. The probability distribution of the $c_{j}$ is most easily obtained by the introduction of characteristic functions.

The characteristic function for the Poisson distribution is

$$
\phi_{\zeta}(t)=\exp \left\{\bar{\zeta}\left(e^{i t}-1\right)\right\} .
$$


where $\bar{\zeta}$ is the expected value of the Poisson-distributed variable $\zeta$. The characteristic function for the random variable $c_{j}$ defined by Eq. (III.78) is

$$
\phi_{c}(t)=\phi_{a}(t) \phi_{b}(-\theta t)=\exp \left\{\bar{a}\left(e^{i t}-1\right)+\bar{b}\left(e^{-i \theta t}-1\right)\right\}
$$

Let us consider the distribution of the quantity $\xi$, where

$$
\xi=\frac{c-(\bar{a}-\theta \bar{b})}{\sqrt{\bar{a}+\theta^{2 \bar{b}}}}
$$

The characteristic function for this variable is

$$
\begin{aligned}
\phi_{\xi}(t) & =\exp \left\{-i t \frac{\bar{a}-\theta \bar{b}}{\sqrt{a+\theta^{2} b}}\right\} \exp \bar{a}\left[e^{i t / \sqrt{\bar{a}+\theta^{2} \bar{b}}-1}+\bar{b}\left[\exp \frac{-i \theta t}{\sqrt{a+\theta^{2} b}}-1\right]\right. \\
& =e^{-t 2 / 2} \exp \left\{-\frac{i t^{3}}{3 !} \frac{\bar{a}-\theta^{3} \bar{b}}{\left(\bar{a}+\theta^{2} \bar{b}\right)^{3 / 2}+\cdots}\right\} .
\end{aligned}
$$

As $\bar{a}$ approaches infinity the characteristic function $\phi_{\xi}(t)$ approaches $e^{-t 2 / 2}$ which is the characteristic function of a variable that is normally distributed with zero mean and unit variance. This is a necessary and sufficient condition that the variable $\xi$, defined in Eq. (III.81), be similarly distributed. This follows from the fact that there exists a one-to-one correspondence between a distribution and its characteristic function. If two distributions are identical, then their characteristic functions are identical and conversely. Thus the corrected counts, Eq. (III.78), are asymptotically normally distributed with mean $\bar{a}_{j}-\theta_{j} \bar{b}_{j}$ and variance $\bar{a}_{j}+\theta_{j} 2 \bar{b}_{j}$.

The above arguments are easily extended to those cases in which the corrected counts $c_{j}$ are obtained from the observed counts $a_{j}$ by 
a series of "background" subtractions. For example, if

$$
c_{j}=a_{j}-\theta_{j} b_{j}-\theta_{j}^{\prime} b_{j}^{\prime}-\ldots
$$

where each of the measured quantities $a_{j}, b_{j}, b_{j}{ }^{\prime}, \ldots .$. are distributed according to a Poisson law with expected values $\bar{a}_{j}, \bar{b}_{j}, \bar{b}_{j}, \ldots$. . respectively, then $c_{j}$ is asymptotically normal with mean value

$$
\bar{c}_{j}=\bar{a}_{j}-\theta_{j} \bar{b}_{j}-\theta_{j} \bar{b}_{j}^{\prime}-\ldots
$$

and variance

$$
\operatorname{var}\left(c_{j}\right)=\bar{a}_{j}+\theta_{j} 2 \bar{b}_{j}+\theta_{j}^{\prime} 2 \bar{b}_{j}^{\prime}+\ldots .
$$

In the above derivations the factors $\theta_{j}$ are assumed to be determined with an error that is negligible compared with that for the $b_{j}$.

\section{Estimates Based on Corrected Spectra}

In terms of counts $c_{j}$ which have been corrected as outlined in Sec. II, the results of the previous subsection show that the quantities

$$
t_{j}=\left(c_{j}-\bar{c}_{j}\right) /\left[\operatorname{var}\left(c_{j}\right)\right]^{I / 2}
$$

are, in the limit of large $c_{j}$, distributed according to the normal law with zero mean and unit variance. Let

$$
Q=\left\{c_{j}: \underline{j} \leqslant j \leqslant \bar{j}\right\}
$$

denote that portion of the corrected simple spectrum that is used to estimate the values of the components of the corresponding parameter vector $\vec{a}$. 
If the counts $c_{j}$ for each channel $j$ are assumed to be statistically independent of the corrected counts in neighboring channels, the quadratic

$$
x^{2}\left(\vec{a}^{\prime}\right)=\sum_{j \in \ell} t_{j}^{2}=\sum_{j \in \ell}\left[c_{j}-F_{j}\left(\vec{a}^{\prime}\right)\right]^{2} / \operatorname{var}\left(c_{j}\right)
$$

is asymptotically distributed as chi-squared provided $\vec{a}^{\prime}$ is chosen such that, for every $\mathrm{j}$ in $\mathrm{C}$.

$$
\bar{c}_{j}=F_{j}\left(\vec{a}^{i}\right)
$$

Equation (III.89) may be considered to be a definition of the "true values" of the vector components $a_{\ell}{ }^{\prime}$.

Let the conditional limiting distribution function of the $c_{j}$ be denoted by $\phi\left(c_{j} \mid \vec{a}\right)$, where

$$
\phi\left(c_{j} \mid \vec{a}\right)=\frac{1}{\sigma_{j} \sqrt{2} \pi} \exp \left\{-\frac{1}{2}\left(\frac{c_{j}-F_{j}(\vec{a})}{\sigma_{j}}\right)^{2}\right\},
$$

with

$$
\sigma_{j}^{2}=\operatorname{var}\left(c_{j}\right)
$$

The probability of observing the result $C$ is

$$
\operatorname{Pr}\{Q\}=\prod_{j \in \ell} \phi_{j}\left(c_{j} \mid \vec{a}\right),
$$

and the maximum-1ikelihood estimates $a_{\ell}$ for the values of $a_{\ell}$ are obtained as the solution of the set of equations

$$
\frac{\partial \ln \operatorname{Pr}\{C\}}{\partial a_{\ell}}=\sum_{j \in C} \frac{c_{j}-F_{j}\left(\vec{a}^{*}\right)}{\sigma_{j}{ }^{2}} \frac{\partial F_{j}\left(\vec{a}^{*}\right)}{\partial a_{l}}=0 .
$$


where

$$
\sigma_{j} 2=F_{j}\left(\vec{a}^{\prime}\right)+\theta_{j} 2 \bar{b}_{j}+\theta_{j}{ }^{\prime 2} \bar{b}_{j}^{\prime}+\cdots \cdots
$$

Except for the additional terms in the expression for the variance, the conditions given by Eq. (III.93) are identical to those defined by Eq. (III.61) in the case in which the background subtractions are treated as nonrandom processes. By analogy with the previous results, Eqs. (III.74) and (III. 76), the error matrix for the joint distribution of the estimates $a_{\ell} *$ is the reciprocal of the real symmetric matrix $B$ whose components are defined as

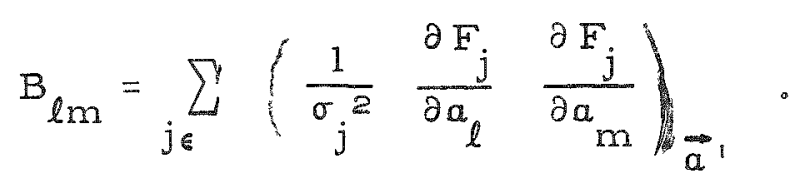

\section{$\underline{\text { Summary }}$}

The results obtained in this section are based on the following major assumptions: (1) The response of the spectrometer to a source of monoenergetic gamma rays can be described by the functions $F_{j}(\vec{a})$ defined in Eq. (II,2). (2) The corrected counts $c_{j}$ are statistically independent for all channel numbers $j_{0}$ (3) There are no random or systematic fluctuations in the channel widths $\Delta_{j}$ for different channels. (4) The "background" subtractions can be carried out, as indicated in Eq. (III.83), with negligible uncertainty in the factors $\theta_{j}$.

The first of these assumptions can be subjected to experimental test and, as discussed in Sec. IV, some effort has been expended to verify the analysis in relation to this assumption. However, since a. separate examination of all of these assumptions is impossible, an attempt was made to take account of these possible perturbations through a normalization of our error estimates by the criterion of external consistency. In 
order to explain the present application of this criterion, we summarize the steps which we followed to obtain the estimates $a_{l}{ }^{*}$ and the associated error matrix.

Let us consider a corrected spectrum $Q$ consisting of data

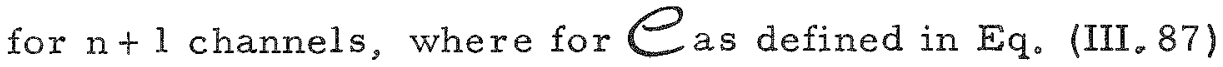

$$
n=\bar{j}-\underline{j} .
$$

The estimates $a_{\ell} *$ are obtained as solutions of the set of equations (II. 12) with $\Lambda_{j} 2$ given by

$$
\Lambda_{j} 2=\sigma 2 / \sigma_{j}^{2}
$$

where $\sigma^{2}$ is a constant, independent of channel number, defined as the weight associated with a measurement of unit variance. In the pth iterative solution of Eqs. (II, 12), $\sigma_{j} 2$ is obtained from the previous iteration as

$$
\sigma_{j} 2=F_{j}(\vec{a} p-1)+\theta_{j} 2 b_{j}+\ldots .
$$

The error matrix is evaluated as the inverse of the matrix $B$, where

$$
B_{l m}=\sum_{j \in e} \Lambda_{j} 2\left(\vec{a}^{*}\right)\left(\frac{\partial F_{j}}{\partial a_{b}} \frac{\partial F_{j}}{\partial a_{m}}\right)_{\vec{a}} .
$$

We have followed the usual procedure of approximating a variance by substituting the sample values $a_{\ell} *$ for the unknown population values $a_{\ell}{ }^{\prime}$. Let the quadratic defined in Eq. (III.88) take on the value $x^{2}\left(\vec{a}^{*}\right)$ for the sample values $a_{l}$. Since this quantity is distributed (at least approximately) as chi-squared with $n-5$ degrees of freedom, its expected value is $n-5$. If the calculated value $\chi^{2}(\vec{a})$ is much greater 
than $n-5$, the experiment itself or the assumptions listed above must be considered suspect. On the other hand, if $\chi^{2}\left(\vec{a}^{*^{*}}\right)$ is much less than $n-5$ the agreement must be considered fortuitous. Thus the normalization is carried out by choosing $\sigma^{2}$ in Eq. (III.97) to be either $\chi^{2}\left(\vec{a}^{*}\right) /(n-5)$ or 1, whichever is larger.

\section{EXPERIMENTAL INVESTIGATION OF THE RESPONSE EUNCTION}

The practical value of the analysis developed in Sec. III depends on the accuracy with which a Gaussian function represents the response of a spectrometer system to monoenergetic gamma radiation. Although the discussion in Sec. III shows that under certain conditions the asymptotic form of the response function is normal, it is necessary to investigate the validity of this approximation for the conditions that actually prevail in an experiment. Obviously any significant difference between the observed response and its functional representation can lead to biased estimates for the values of the parameters of the assumed function and consequently to systematic errors in determinations of gammaray energy and intensity。

A number of the results derived in Sec. III can be subjected to direct experimental test. We now consider three specific examples, namely, (i) the representation of the shape of observed response by the Gaussian function, Eq. (III. 47); (ii) the energy dependence of the width of the response curve, Eq. (III.43); and (iii) the relation between the mean pulse height of a line and the energy of the associated gamma radiation, Eq. (III.31).

As noted earlier our primary interest in these investigations is the practical one of establishing realistic limits for the accuracy that can be attained in various energy and intensity measurements with a crystal spectrometer. However, it is possible that some of the present 
techniques could be used in the investigation of some of the remaining questions concerning the mechanism of energy conversion and transfer in crystals.

\section{The Line Shape}

A large amount of data for gamma rays in the energy interval from $100 \mathrm{kev}$ to $3 \mathrm{Mev}$ has been analyzed as described in Secs. II and III. For each full-energy peak, the minimum value of $\Omega$ was computed from Eq. (II.3) and compared with the expected value of the corresponding chi-squared distribution. Examples of the results of these calculations are shown in Figs。 $9(a)$ and $9(b)$ For the relatively small sample represented by these data, the agreement must be considered satisfactory and indicates that there is no signuficant difference between the shape of a Gaussian function and the measured shape of an instrumental line. (By "significant" in this context we mean that the observed deviations are generally smaller in magnitude than the random variations which must be expected from ordinary counting statistics.) In each of these comparisons the observed line was truncated such that the extreme channel numbers $x_{e}$ saisfy the inequality

$$
\left|x_{e}-a_{2}\right| \geq 1.6 a_{3}
$$

where $a_{2}$ and $a_{3}$ are defined by Eq. (II.2). This condition guarantees that at least $97 \%$ of the counts in the full-energy peak are included in the analyzed data。

As shown in Sec. III, the vanishing of the coefficient of skewness $\gamma_{q}^{(j)}$ is a condition which must be satisfied if the response function is Gaussian. We have analyzed several observed lines by use of an Edgeworth distribution [Eq. (III.49) with $\gamma_{q}^{(2)}$ set equal to zero] in place of the Gaussian function in the first term of Eq. (II.2). The values of 


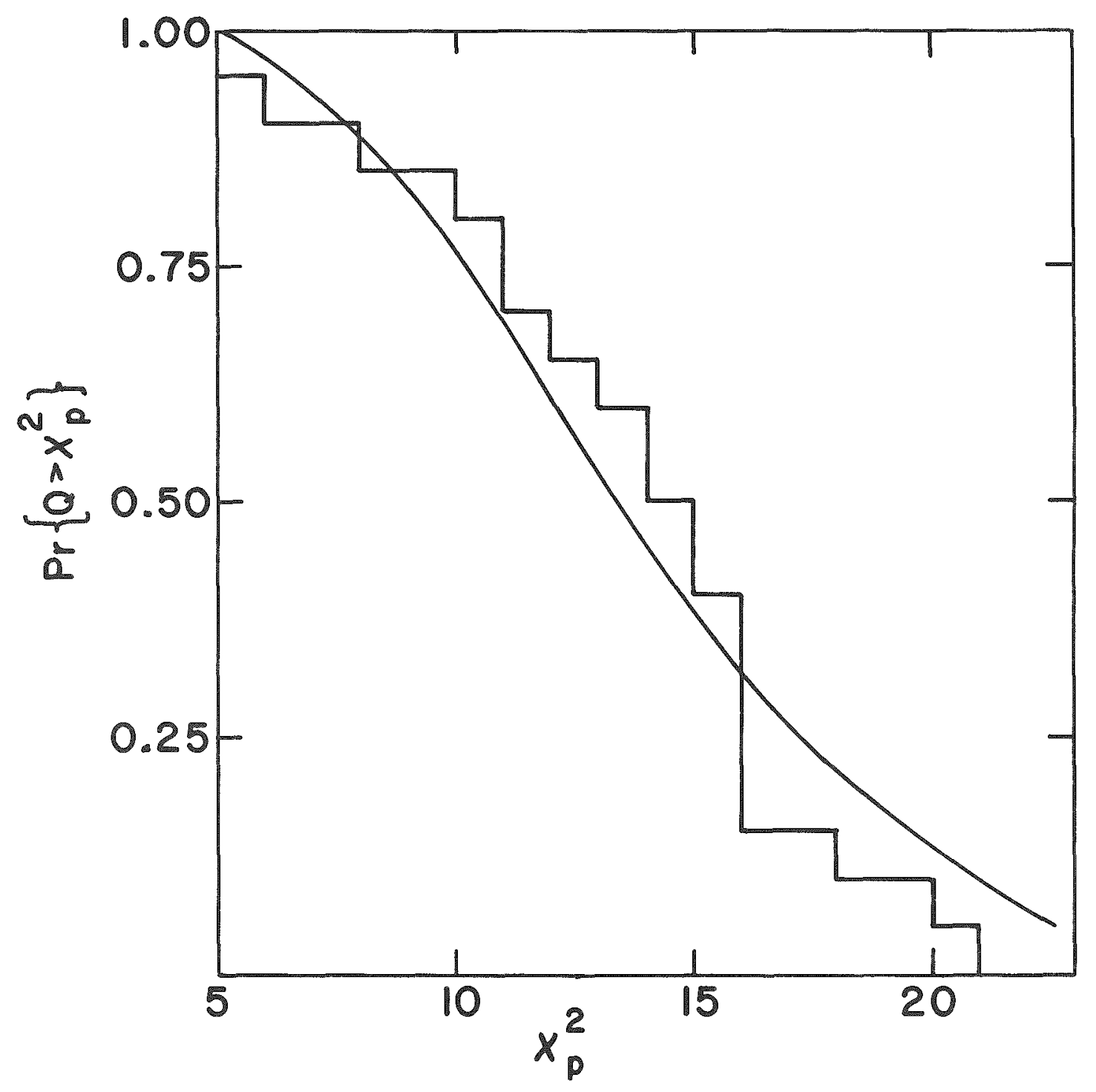

Fig. 9(a). A comparison of the observed distribution of min $(Q)$ and the theoretical chi-squared distribution. The histogram shows the distribution of the minimum values of $Q$, Eq. (II.3), calculated for 20 response curves at an energy of 2.1 Mev. The data were grouped in 19 channels. The continuous curve is the corresponding chi-squared distribution for 14 degrees of freedom. 


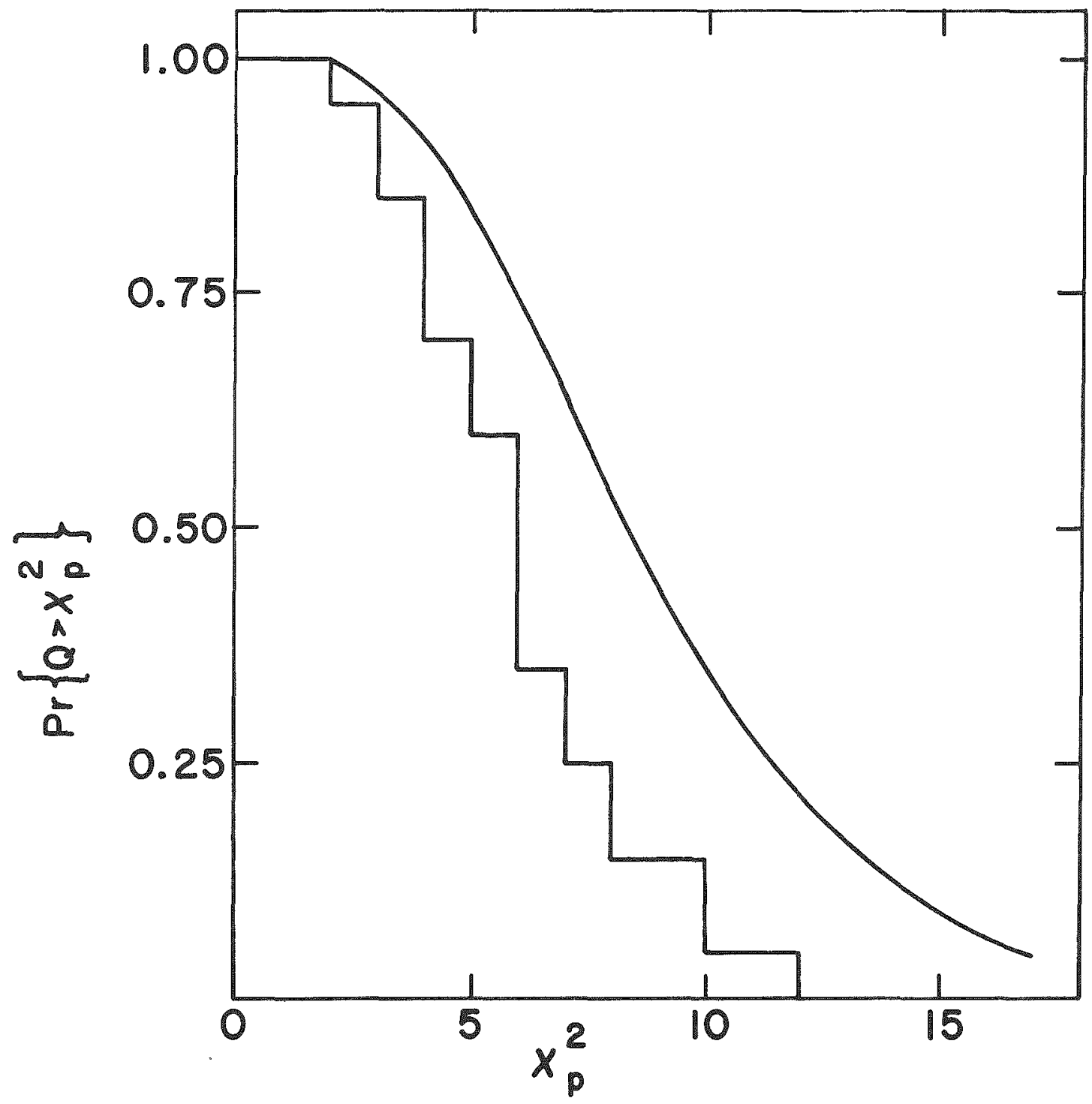

Fig. 9(b). A comparison of the observed distribution of min $(Q)$ and the theoretical chi-squared distribution for 20 response curves at an enerby of 1.0 Mev. The data were grouped in 14 channels so that the corresponding chi-squared distribution has 9 degrees of freedom. 
$\gamma_{q}$

(1) which were obtained are shown in Fig, 10, Obviously very little conjecture concerning the behavior of this coefficient is justified on the basis of these results. However, it should be emphasized that no special effort was made to obtain data of sufficient statistical accuracy to carry out this analysis. In fact the lines that were analyzed in this manner contained less than twice the number of counts routinely taken in an experiment. A more precise determination, particularly of the asymptotic behavior of this coefficient, would be of some interest.

For our present purpose we observe that the se results indicate a small positive skewness in the observed line shape. However, at least for the number of counts usually obtained, the resultant deviation from a Gaussian shape is less in magnitude than the random fluctuations which can be attributed to counting statistics. The possibility that a nonzero coefficient of skewness introduces a bias in our estimates of mean channel positions is considered below。

\section{The Line Width}

The energy dependence of the relative variance $\mathcal{U}_{\mathrm{q}}$ of the response line, as predicted by Eq。(III.43), was investigated by plotting the observed values of $\mathcal{U}_{\mathrm{q}}$ against the reciprocal energy $\mathrm{E}^{-1}$ of the incident gamma ray. The results are shown in Fig. 11. The break in the predicted linearity of this curve occurs in an energy interval, roughly from $200 \mathrm{kev}$ to $400 \mathrm{kev}$, where the Compton cross section for NaI becomes larger than the photoabsorption cross section. The region of nonlinearity is thus a region of transition from predominantly single to predominantly multiple excitation processes within the crystal.

Similar measurements have been reported by Bisi and Zappa. ${ }^{7}$ They also observe a break from linearity, but at a somewhat higher energy. Furthermore, their results show an increasing deviation from linearity with increasing energy of the incident gamma ray。 On the 


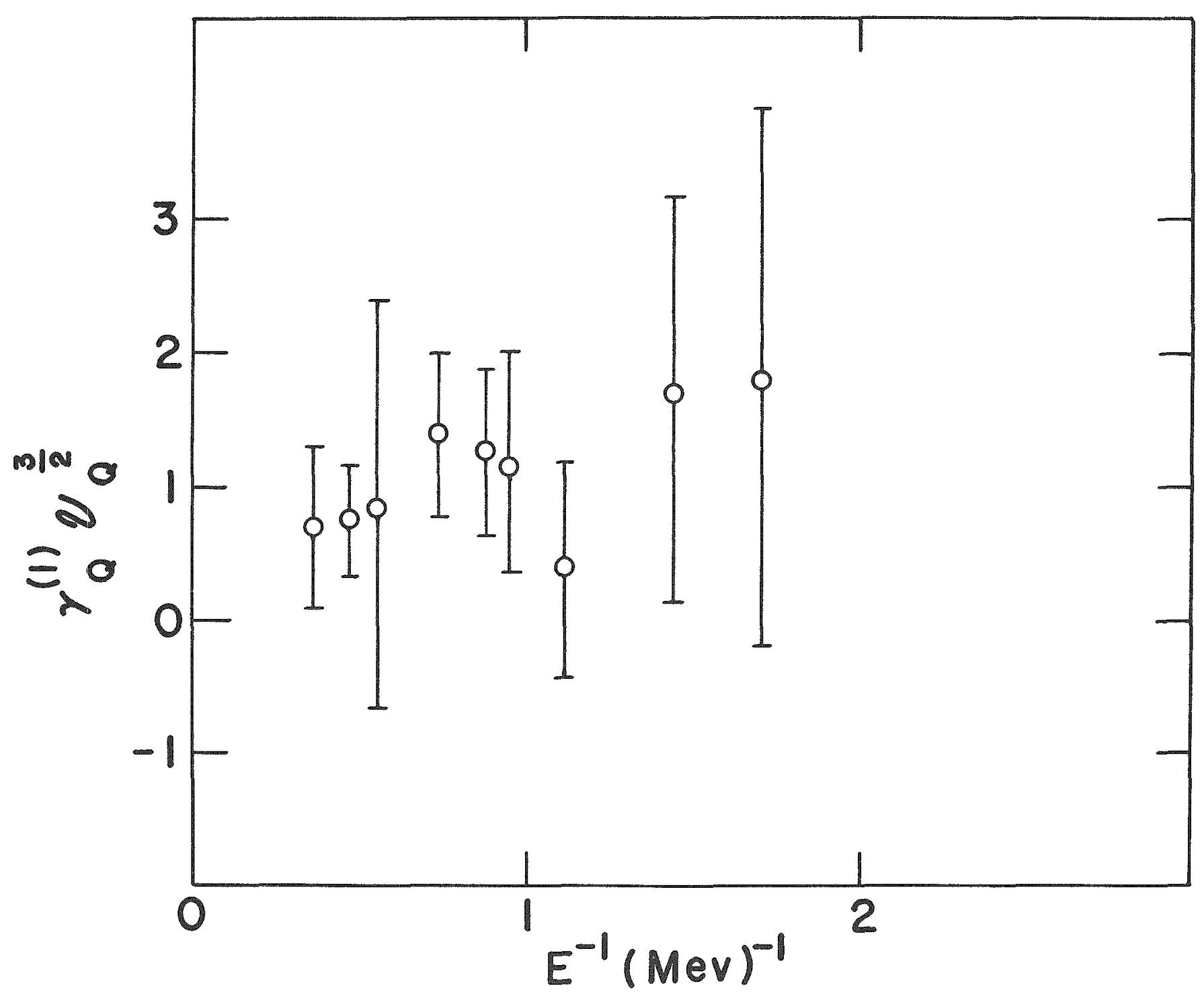

Fig. 10. The observed values of $\gamma_{q}^{(1) / / J_{q}^{3 / 2}}$ as a function of $1 /$ E. The conversion factor between pulse-height and energy is not included in the values of $\gamma_{q}^{(1) /)^{3 / 2}}$ shown in this graph.

a 


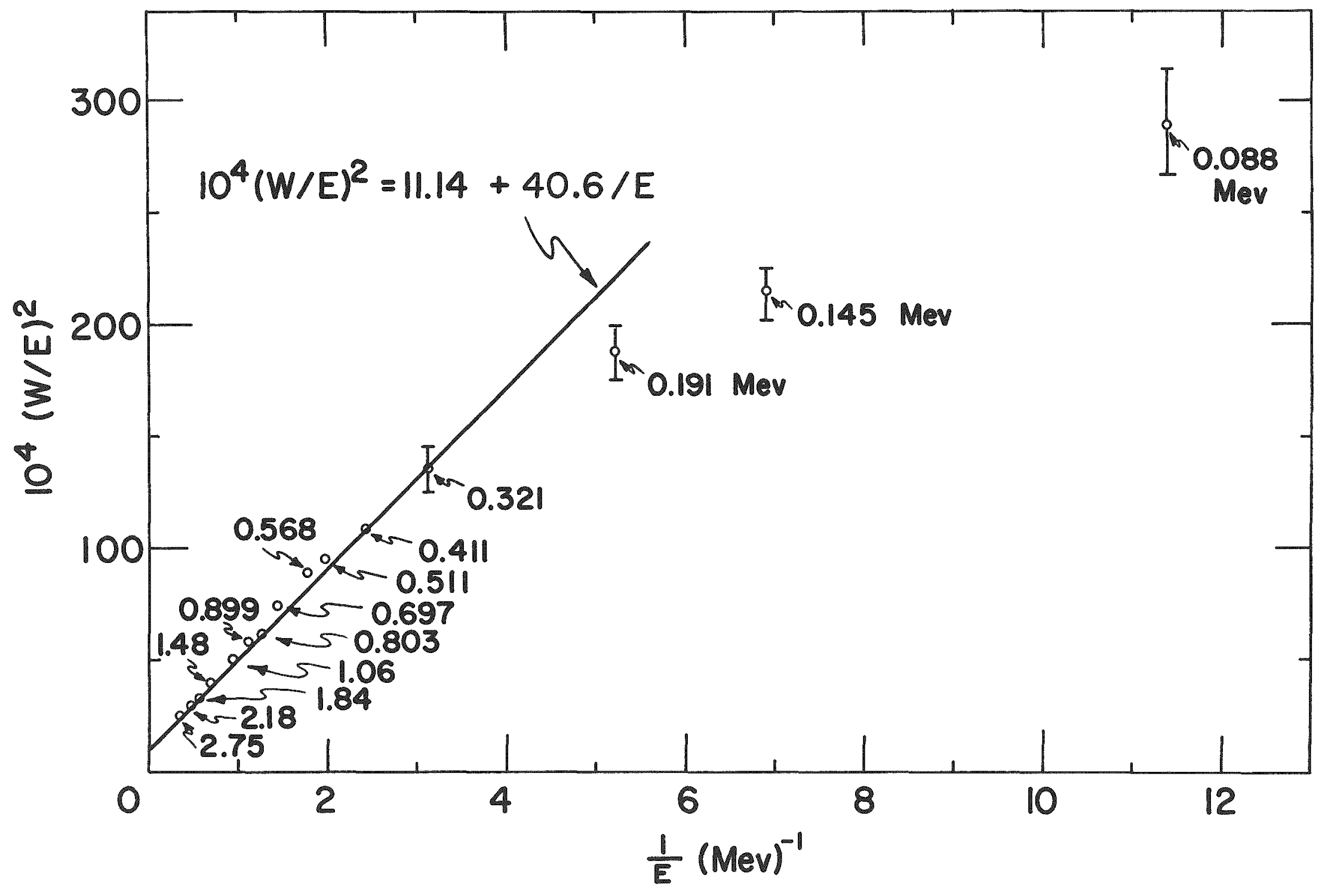

Fig. 11. The observed values of $\mathbb{Q}_{\mathrm{q}}$ as a function of $1 / \mathrm{E}$. The quantity $(W / E)^{2}$ is the square of the ratio of observed width to energy of a response line. This is proportional to the relative variance $\mathcal{1}_{q^{\circ}}$ 
other hand our results, which cover the same range of incident energies, are in good agreement with a linear law for all energies outside of the transition region mentioned above.

In order to explain the results of their measurements Bisi and Zappa assume that, insofar as the contribution to the photopeak at $E$ is concerned, an event giving rise to a Compton electron with energy $E_{1}$ and a photoelectron with energy $\mathbf{E}-E_{1}$ can be treated as the simultaneous absorption in the crystal of two independent quanta with energies $E_{1}$ and $E-E_{I}$. It is of interest to consider a derivation of the expression given by these authors for the relative variance of the pulse-height distribution in this latter case.

We use the notation introduced in Sec. III, where $x_{i}$ is now the number of light quanta produced in the phosphor for a gamma ray of energy $E_{i}$. The number $\int_{x}$ of photoelectrons that arrives at the first dynode as a result of the incidence of two gamma rays with energy $E_{\perp}$ and $E-E_{1}=E_{2}$ is

$$
\mathscr{d}_{\mathrm{x}}=\sum_{k=1}^{\mathrm{x}_{1}} \mathrm{t}_{\mathrm{k}} \mathrm{q}_{\mathrm{k}}+\sum_{\mathrm{k}=1}^{\mathrm{x}_{2}} \mathrm{t}_{\mathrm{k}} \mathrm{q}_{\mathrm{k}}
$$

The resulting output pulse $q$ is

$$
q=\sum_{i=1}^{d_{x}} q_{i}
$$

The moment-generating function for the $q$ distribution of this process becomes

$$
\Omega_{q}(k)=\sum_{i, j} q_{i} q_{j} \exp \left\{\left(\bar{x}_{I} t_{i}+\bar{x}_{2} t_{j}\right)\left(e^{\psi} m(k)-1\right)\right\}
$$

and the corresponding semi-invariant of order two is 


$$
\begin{gathered}
\lambda_{q}^{(2)}=\left(\bar{x}_{1}+\bar{x}_{2}\right) \lambda_{t}{ }^{(I)}\left[\left(\lambda_{m}^{(I)}\right)^{2}+\lambda_{m}{ }^{(2)}\right]-\left(\bar{x}_{I}+\bar{x}_{2}\right)\left[\lambda_{m}^{(I)} \lambda_{t}^{(I)}\right]^{2} \\
+\left(\lambda_{m}^{(1)}\right)^{2} \sum_{i, j} q_{i} q_{j}\left[\bar{x}_{I} t_{i}+\bar{x}_{2} t_{j}\right]^{2} .
\end{gathered}
$$

If we assume that the two quanta are absorbed in completely uncorrelated events, the double sum in the last term on the right-hand side of Eq. (IV.5) can be evaluated to give

$\sum_{i, j} q_{i} q_{j}\left[\bar{x}_{1} t_{i}+\bar{x}_{2}{ }_{j}\right]^{2}=\bar{x}_{I} 2\left(\lambda_{t}{ }^{(I)}\right)^{2}+\bar{x}_{2} 2\left(\lambda_{t}{ }^{(I)}\right)^{2}+2 \bar{x}_{1} \bar{x}_{2}\left(\lambda_{t}{ }^{(I)}\right)^{2}$,

and the associated relative variance is

$$
U_{q}=\mathcal{L}_{t}\left[1-\frac{2 \bar{x}_{I} \bar{x}_{2}}{\left(\bar{x}_{1}+\bar{x}_{2}\right)^{2}}\right]+\frac{1+\sum_{m}}{\bar{x} \lambda_{t}^{(1)}} \text {. }
$$

Using Eq. (IV. 7) with $\bar{x}_{I}$ proportional to the average energy of the Compton electron and considering that a fraction of the pulses that contribute to the full-energy peak at $\mathrm{E}$ arise from photoabsorption, Bisi and Zappa calculate values of the relative variance which agree quite well with their measured values.

The data shown in Fig. 11 do not exhibit the curvature at higher energies which is predicted by Eq. (IV.7) and which is observed by Bisi and Zappa. The present data were obtained with a considerably larger crystal than was used by Bisi and Zappa. Thus for incident gamma rays of given energy, a larger fraction of multiple-excitation processes should contribute to our full-energy peak and a correspondingly larger deviation from linearity with increasing energy would be predicted by Eq. (IV. 7).

For crystals of the size used in the present experiment the multiple events contributing to the full-energy peak are about equally divided between those that involve three or more collisions in the crystal and those 
that involve two collisions for incident energies above about $0.7 \mathrm{Mev}$. These excitation processes of higher multiplicity should be considered in the calculation of the variance $\mathcal{W}_{\mathrm{q}}$. In the case of three statistically independent events which contribute to the full-energy peak, the relative variance of the $q$ distribution becomes

$$
\mathscr{L}=\mathscr{U}_{t}\left[1-2 \frac{\left.\bar{x}_{1} \bar{x}_{2}+\bar{x}_{1} \bar{x}_{3}+\bar{x}_{2} \bar{x}_{3}\right]}{\left.\left(\bar{x}_{1}+\bar{x}_{2}+\bar{x}_{3}\right)^{2}\right\rfloor}+\lambda_{t}^{(1)} \frac{1+\mathcal{L}_{m}}{\left(\bar{x}_{1}+\bar{x}_{2}+\bar{x}_{3}\right)}\right. \text {. }
$$

The numerical calculation of variances when these higher order processes are considered requires knowledge not only of the relative contribution to the full-energy peak from each of these processes but also of the distribution of energies at each stage of a given multiple event. We have not carried out such a calculation.

However, from Eq. (IV.8), it seems likely that processes of higher multiplicity would further increase the curvature of $\mathcal{U}_{\mathrm{q}}$ as the energy of incidence is increased. If this is true the dependence of $\mathcal{L}_{\mathrm{q}}$ on energy, shown in Fig. 11, is not explained by Eqs. (IV.7) and (IV.8).

The assumption of complete statistical independence of events in a Compton process, which is necessary for the application of Eq. (IV.7), is difficult to justify in these calculations. However, the apparent existence of a well-defined region of nonlinearity, which is also a region of transition from single-to multiple-excitation processes, suggests that some effect such as that suggested by Bisi and Zappa is contributing to the observed variance. One possibility is the following purely qualitative explanation. If we drop the assumption that the number $\mathrm{x}$ of light quanta resulting from a gamma ray of given energy E follows a. Poisson distribution, then the relative variance of the response line becomes

$$
1_{q}=\mathcal{L}_{t}+\left(1+\mathcal{D}_{t}\left(\mathcal{L}_{\mathrm{x}}-\frac{1}{\mathrm{x}}\right)+\frac{\left(1+\mathcal{N}_{\mathrm{m}}\right)}{\lambda_{\mathrm{t}}^{(1)} \overline{\mathrm{x}}}\right. \text {. }
$$


where $\mathcal{L}_{x}$ is the relative variance of $x$. If for single-excitation processes $\mathcal{H}_{\mathrm{x}}$ decreases with increasing energy, Eq. (IV.9) would predict roughly the energy dependence of $\mathcal{D}_{\mathrm{q}}$ which is observed in Fig. 11. For incident energies above the transition region, the main contribution to $\mathcal{D}$ would be from the lower energy events in multiple processes and these would show a much less pronounced dependence on the incident energy than would the singleexcitation processes. It is also quite probable that more complicated energy dependences of $\mathcal{L}_{t}$ and $\mathcal{L}_{\mathrm{m}}$ are involved. Obviously these questions require further investigation.

The Energy Calibration of the Spectrometer: The Two-Error Problem

It is well known that the size of the output pulse from a scintillation counter is closely proportional to the energy of the incident gamma ray. This is also the prediction of Eqs。(III, 31) and (III.412). However, because the labeling of the pulse-height channel corresponding to zero energy is arbitrary, Eq. (III.4la) must be written in the form

$$
E=a+b a_{2}
$$

where $a_{2}$ is the channel number for the mean pulse height of the full energy peak at energy $E, a$ is the energy corresponding to channel number zero, and, in terms of the notation of Sec. III,

$$
b=1 /\left(\zeta \lambda_{t}^{(1)} \lambda_{m}^{(1)}\right)
$$

In our investigation ${ }^{\ominus}$ of the relation between pulse size and energy in the interval from $500 \mathrm{kev}$ to $3000 \mathrm{kev}$, we find that the specific scintillation $\mathrm{da}_{2} / \mathrm{dE}$ is not constant, as would be predicted by Eq. (IV.10) provided $b$ is independent of energy, but rather varies as

$$
\frac{d a_{2}}{d E} \approx \frac{1}{A-B E}
$$


where $\mathrm{B} / \mathrm{A} \leqq 10^{-5} / \mathrm{kev}$. In fact, when the spectrometer is calibrated with a set of standard gamma-ray energies in the energy interval from $500 \mathrm{kev}$ to $3000 \mathrm{kev}$ it is found that this calibration gives in the region of about $2000 \mathrm{kev}$ a measure of the energy about 0.5 percent higher than a simple linear calibration between two standards at $1400 \mathrm{kev}$ and $2750 \mathrm{kev}$.

A partial explanation for this observed nonlinearity is the fitting procedure of Sec. III, which is used to estimate the value $a_{2}$ of the mean position. If the full-energy peak is a skewed distribution, $a_{2}$ will be somewhere between its mean $\bar{q}$ and its mode $q_{0}$. These statistics are related by the coefficient of skewness $\gamma_{I}$ as

$$
\bar{q}=q_{0}-\gamma_{1} a_{3} / \sqrt{8}
$$

where $a_{3}$ is the width of the distribution as defined in Eq. (II. 2). If we assume that it is $q_{0}$ instead of $\bar{q}$ which is actually being interpreted as $a_{2}$ in Eq. (IV. 10), then for $\gamma_{I}>0$ the specific scintillation would show an apparent decrease with increasing energy as predicted by Eq. (IV.12). However, from the results of the analysis by means of the Edgeworth series we find that the upper limit of deviations from linearity, i。e., deviations from constant slope, as a result of this effect is about half of the deviation actually observed. This result is in qualitative agreement with similar considerations published by Wright ${ }^{10}$ for much lower incident energies.

Apparently the observed nonlinearity cannot be attributed entirely to the method of analysis and it is necessary to conclude that this is a real property of the scintillator system. Several plausible mechanisms have been suggested ${ }^{3}, I I$ to explain the decrease of $\mathrm{da}_{2} / \mathrm{dE}$ with increasing values of $E_{\text {. Th }}$ Thus far, however, it has not been possible to predict the energy dependence of the coefficient b in Eq. (IV.11) from these "models." It is hardly necessary to point out that this constitutes the main limitation on the use of a crystal spectrometer in the determination of energy. 
As an interpolation formula for the energy corresponding to a given mean pulse height we have used the relation

$$
E=a+b a_{2}+c a_{2}{ }^{2}
$$

The only a priori justification for a relation of this form is that it provides a first approximation to Eq. (IV.13). However, we have found that the inclusion of the quadratic term in Eq. (IV.14) improves the energy calibration of the pulse height to an extent greater than would be expected by the introduction of an additional term which is completely arbitrary. A discussion of the data on which this statement is based is given in the next subsection.

In the energy calibration of the pulse-height scale, we are given the structural relation (IV. 14) and a set of $n$ measurements $\left(E_{j}, a_{j}\right)$ where $a_{j}$ is the mean pulse height corresponding to an incident energy $E_{j}$. (In the remainder of this section we drop the subscript "2" in the designation of mean pulse height.) The problem is to estimate the values of the coefficients in Eq. (IV, 14) from the se data.

This problem differs from the usual estimation problem in that both "coordinates" $E_{j}$ and $a_{j}$ are measured quantities and as such are subject to errors of observation. The general problem of estimation under such conditions has been discussed by Wald. ${ }^{12}$ In the present case, in which the variances $\operatorname{var}\left(a_{j}\right)$ and $\operatorname{var}\left(E_{j}\right)$ of the measured quantities are assumed to be known, the general principle of least squares gives an efficient and unbiased estimate of the values of the coefficients in the structural relation. An outline of the general principle of least squares is given in the Appendix.

In the present case the principle of least squares requires that the estimated values $e_{j}$ and $a_{j}$ be determined from the measured values $E_{j}$ and $a_{j}$ such that the quantity

$$
S=\sum_{i=1}^{n}\left\{\frac{\left(e_{i}-E_{i}\right)^{2}}{\operatorname{var} E_{i}}+\frac{\left(a_{i}-a_{i}\right)^{2}}{\operatorname{var} a_{i}}\right\}
$$


be a minimum subject to the conditional equations

$$
e_{i}-a-b a_{i}-c a_{i} 2=0, \quad i=1,2, \ldots, n 。
$$

Deming $^{13}$ has shown that the minimum value of $S$ is distributed as chisquared provided that the observations are random and that Eqs. (IV.16) are the correct relations for the true values of the coordinates. The number of degrees of freedom of the chi-squared distribution is given as the number of observed pairs minus the number of structural-equation parameters whose values are to be estimated.

The method of least squares is particularly convenient in the present problem since it is the only known method of adjustment in the related problem of energy determination which is considered in the next section.

We return to the point mentioned at the end of the previous subsection. A calibration of the pulse-height scale over the energy interval from 0.51 Mev to 2.75 Mev was made according to the principle of least squares for five "standard" energies $E_{i}$. Hence the number of degrees of freedom for the theoretical distribution of $S$ is 2 in the case of quadratic interpolation and 3 in the case of linear interpolation. For a series of eight such determinations, the computed values of $S$ varied from about 3 to 20 for quadratic interpolation and from 30 to 80 for linear interpolation. The average ratio of values of $S$ for these two calibration functions is 5.9. If equal validity is assumed for both interpolation formulae, the expected value of this ratio is 1.5. This indicates that the quadratic term in Eq. (IV.14) improves the agreement to an extent greater than would be expected if this term represented an arbitrary free parameter.

Even for quadratic interpolation, however, the distribution of observed values of $\mathrm{S}$ does not agree with the theoretical chi-squared 
distribution as well as might be expected if Eq. (IV.14) were an accurate representation of the relation between energy and pulse height.

\section{EXPERIMENTAL TESTS FOR CONSISTENCY}

On the basis of the experimental results which have been discussed thus far, it is not possible to state with assurance that the as sumptions associated with the analysis are valid to within the statistical uncertainties expected in a given measurement. It thus remains to determine whether the analysis provides realistic estimates of the accuracy of a given result. Fortunately it is possible to test the analytic error esti= mates in both energy and intensity measurements by means of well established physical principles, namely the laws of conservation of energy and of the exponential decay of a radioactive source.

\section{Measurement of Energy}

The transitions from the 2.18-Mev state to the ground state in NdI4, shown in Fig. 4, provide a consistency check on the estimated accuracy of our energy measurements. If the small difference in recoil energy is neglected, the sum of the energies of the two transitions in cascade to the ground state must equal the energy of the crossover transition. We have measured ${ }^{\ominus}$ the energies of the cascade gamma rays to be 1487.0 $01.1 \mathrm{kev}$ and $696.7 \pm 0.6 \mathrm{kev}$, and the energy of the crossover transition to be $2186.0 \pm 2.2 \mathrm{kev}$. This latter value is to be compared with the sum of the energies of the cascade gamma rays, which is $2183.7 \pm 1.7 \mathrm{kev}$. If the difference between these results is treated as a sample drawn from a normal population with zero mean, it is easily shown that 4 out of 10 similar experiments will result in a difference at least as large as that observed.

A description of these measurements has been published. However, some of the details of the analysis which were not included in the published work deserve mention. 
The problem of energy calibration differs fromthe problem of energy determination in that for the latter case one of the energy values, say $E_{n+1}$, is considered as an unknown. In order to indicate how the problem of energy determination is treated, suppose that the pairs $\left(E_{j}, a_{j}\right)$ for $j=1,2, \ldots$. . n have been measured as has the mean pulse height $a_{n+1}$ corresponding to an energy value $E_{n+1}$ which we wish to determine. Let the quadratic $S$ be defined as

$$
S=\sum_{i=1}^{n}\left(e_{i}-E_{i}\right) 2 / \operatorname{var}\left(E_{i}\right)+\sum_{i=1}^{n+1}\left(a_{i}-a_{i}\right) 2 / \operatorname{var}\left(a_{i}\right)
$$

In this case the principle of least squares requires that $S$ be minimized subject to the conditions

$$
e_{i}-a-b a_{i}-c a_{i}{ }^{2}=0, \quad i=1,2, \ldots, n+1,
$$

where $e_{n+1}$ as well as $a, b$, and $c$ are now considered as unknown parameters. A method for the solution of this problem is given in the Appendix.

A further complication is introduced by the following experi mental situation which frequently arises in practice. The energy values, say $E_{n+1}$ and $E_{n+2}$, axe determined in terms of $n$ "standard" energies $E_{j}$ $j=1,2, \ldots \ldots, n$, as in Eqs. $(V, 1)$ and $(V, 2)$. Then a subset of the original "standards," say $E_{j}, j=1,2, \ldots, n-2$, are used in conjunction with the values $E_{n+1}$ and $E_{n+2}$ to determine a new energy value $E_{n+3^{\circ}}$. Let the original conditional equations be

$$
e_{j}-a-b a_{j}-c a_{j} 2=0, \quad j=1,2, \ldots, n+2,
$$

where $a, b, c, e_{n+1}$, and $e_{n+2}$ are parameters whose values are to be estimated; and let the conditional equations for the second measurement be

$$
e_{i}-a^{\prime}-b^{\prime} \beta_{i}-c^{\prime} \beta_{i}^{2}, \quad i=1,2, \ldots, n-2, n+1, n+2, n+3,
$$


where $a^{\prime}, b^{\prime}, c^{\prime}$, and $e_{n+3}$ are parameters whose values are to be estimated. Obviously, the equations of this second set are not observationally independent since $e_{n+1}$ and $e_{n+2}$ are determined only in terms of the re maining $e_{i}$ in Eqs. $(V .3)$. However, there is a related set in which the $2 n+1$ equations in the seven unknowns $a, b, c, a^{\prime}, b^{\prime}, c^{\prime}$, and $e_{n+1}$ namely,

$$
\begin{array}{ll}
e_{j}-a-b a_{j}-c a_{j}{ }^{2}=0, & j=1,2, \ldots, n, \\
e_{i}-a^{\prime}-b^{\prime} \beta_{i}-c^{\prime} \beta_{j} 2=0, & i=1,2, \ldots, n-2, n+3,
\end{array}
$$

and

$$
a+b a_{j}+c a_{j}{ }^{2}-a^{\prime}-b^{\prime} \beta_{j} 2=0, \quad j=n+1, n+2 。
$$

are observationally independent and can be treated in the same manner as described above.

The least-squares formalism described in the Appendix is applicable to the case in which the conditional equations are not independent. However, in this case the weight matrix, Eq, (A.14), is not diagonal and the computation becomes somewhat lengthy。

\section{Measurement of the Lifetimes of Radioactive Nuclei}

In order for a counter to register an incident gamma ray, it is necessary that no gamma ray has arrived at the counter during a preceding time interval of length $\tau$. This interval of time during which the counter is unable to register an incident pulse is called the resolving time, or dead time, of the counting system. For a system which includes a multi-channel analyzer of the type used in the present measurements. this resolving time depends linearly on the energy $\epsilon$ of the recorded pulse, i.e.,

$$
\tau_{\epsilon}=\tau_{0}+k \in
$$


where $\tau_{0}$ and $K$ are constants. If the radioactive source is such that the counting rate for the entire measured spectrum exceeds $\sim 10^{3}$ counts $/ \mathrm{sec}$. it is necessary to take explicitly into account this finite resolving time in any determination of relative intensities and, consequently, in any measurement of the lifetime of a radioactive decay. This can be done in a manner similar to that proposed by Schiff. ${ }^{14}$ Several features of the present measurements, however, make it desirable to reconsider this problem in some detail.

Let $\Lambda_{\epsilon}(t)$ denote the expected rate at which gamma rays of energy $\epsilon$ are incident on the sensitive portion of the detector. For a radioactive source which emits gamma rays of energy $\epsilon$ with decay con$\operatorname{stant} \lambda_{\epsilon}$ we have

$$
\Lambda_{\epsilon}(t)=\beta_{\epsilon}+N_{\epsilon} \lambda_{\epsilon} e^{-\lambda \epsilon t},
$$

where $\beta_{\epsilon}$ is a constant (with respect to the time $t$ ) background rate and $N_{\epsilon}$ is a constant that depends on the strength of the source for emission of gamma rays of energy \& at some arbitrary zero time and on the geometrical arrangement of the experiment. The expectation that a gamma ray with energy $\epsilon$ arrives at the counter in the time interval $t-T$ to $t$ is

$$
G_{\epsilon}\left(t-\tau_{\epsilon}, t\right)=\int_{t-\tau_{\epsilon}}^{t} d t \Lambda_{\epsilon}(t) .
$$

If the distribution of the number of gamma rays with energy $\epsilon$ incident on the counter in any time interval is Poisson, then the chance at time that no such gamma ray has arrived at the counter during the preceding inter val of duration $\tau_{\epsilon}$ is $\exp \left\{-G_{\epsilon}\left(t-\tau_{\epsilon}, t\right)\right\}$.

Suppose we wish to determine the value of the decay constant $\lambda_{E}$ corresponding to a particular gamma-ray energy $E$ contained in the spec-

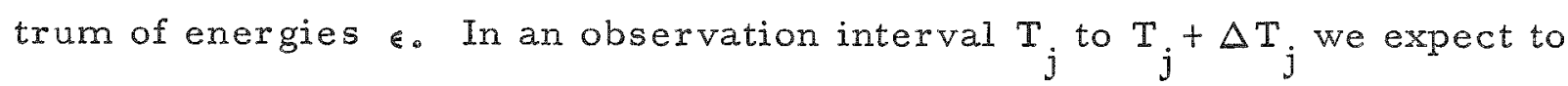
count a number $n_{j}(E)$ of these gamma rays where 


$$
n_{j}(E)=\int_{T_{j}}^{T_{j}+\Delta T_{j}} d t \Lambda_{E}(t) \exp \left\{-\sum_{\epsilon} G_{G}\left(t-T_{\epsilon}, t\right)\right\},
$$

The summation on $\epsilon$ in Eq. $(V .9)$ is over the entire observed spectrum and it has been assumed that the decays corresponding to different values of $\epsilon$ are independently distributed. In terms of the values $a_{1 j}$ and $a_{3 j}$ of the first and third components of the vector $\vec{a}$ estimated, as discussed in Sec. II, for the photopeak at energy $E$ we have

$$
n_{j}(E)=\sqrt{\pi}_{1 j} a_{3 j}+b_{j}(E)
$$

where

$$
b_{j}(E)=\beta_{E} \int_{T_{j}}^{T_{j}+\Delta T_{j}} d t \exp \left\{-\sum_{\epsilon} G_{\epsilon}\left(t-T_{\epsilon}, t\right)\right\}
$$

is the number of background counts in the observed spectrum at energy $\mathbb{E}_{\sigma}$

Let $\mathrm{d}_{\mathrm{j}}$ denote that fraction of the $\mathrm{jth}$ counting interval during which the counting system is in a state such that it is not capable of registering an incident gamma ray。 Most multi-channel pulse-height analyzers record a separate measure of this total dead time $d_{j} \Delta T_{j}$ For reasonably small values of $d_{j}$ we have

$$
\mathrm{d}_{j} \Delta \mathrm{T}_{\mathrm{j}}=\sum_{\epsilon} \mathrm{n}_{\mathrm{j}}^{\mathrm{o}}(\epsilon) \tau_{\epsilon}
$$

where $n_{j}{ }^{\circ}(\epsilon)$ is the number of gamma rays of energy 6 which would have been counted in the jth interval if the dead time of the counter were zero.

If a given spectrum consisting of $M$ separate full-energy peaks is measured for $L$ separate intervals $\Delta T_{j}$, there are ML equations of the form (V.12) which describe the results of the experiment. For each of the $M$ values of e corresponding to a full-energy peak there are at most, two "unknowns" $N_{\epsilon}$ and $\lambda_{\epsilon}$. If the values of $\tau_{0}$ and $\kappa$ in Eq。 $(V .6)$ are also 
to be determined from the data, we have $L(M+1)$ conditional equations which contain at most $2(\mathrm{M}+1)$ unknowns. Equations $(V .9)$ and $(V .12)$ thus constitute an overdetermined set for $L>2$. However, for a complex spectrum consisting of many photopeaks the solution of this set of equations becomes involved and requires a series of decisions which are impractical to code for a digital computer. For example, the value of each $\beta_{\epsilon}$ in Eq. $(V .9)$ must be obtained from the observed $b_{j}(\epsilon)$ by solving $E q .(V .11)$. Also the factor $\Lambda_{E}(t)$ in $E q .(V .9)$ must include as background not only the natural background rate $\beta_{E}$ but also any contribution from the tails of neighboring photopeaks. Variations in the decay rate of these additional backgrounds prevent their inclusion in the constant term $\beta_{E^{\circ}}$ This is also true for each of the terms $\Lambda_{\epsilon}(t)$ contained in the factors $G_{\epsilon}$ in Eq. $(V .9)$. For these terms, however, there is the additional complication that the resolving time must be properly correlated with the observed pulse height for each of the backgrounds.

For all practical purposes it is obvious that such complications must be avoided in order to obtain a measure of the decay constant of any radioactive scurce. This imposes a limitation either on the maximum countung $x$ ate in any experiment or on the complexity of the spectra which can be measured. These two limitations actually are not independent. For a spectrum containing many photopeaks the counting rate in the peak of interest may be a small fraction of the total counting rate. If this latter is limited to less than $10^{3}$ counts/sec it may be impossible to obtain a number of counts in the photopeak during a practical counting interval that are sufficient for statistically significant results. However, it is usually possible to design the experiment such that enough of these complications are avoided that the analysis becomes practical.

For example, suppose that the decay constant $\lambda_{E}$ corresponding to radiation of energy $\mathbb{E}$ is to be measured. Let the energy values $\epsilon$ of the various photopeaks in the measured spectrum be divided into two sets 
such that one set $\varepsilon$ contains all peaks of energy $\epsilon$ with $\lambda_{E}=\lambda_{E}$ and a second set $\varepsilon^{\prime}$, the complement of $\mathcal{E}$, contains the remaining photopeaks, For all $\epsilon$ in $\varepsilon^{\prime}$ assume that $\lambda_{\epsilon}$ is sufficiently less than $\lambda_{E}$ that the variation in source strength for these gamma rays can be neglected over any single observation interval $\Delta T_{j}$. This implies for all observations $j$ and for all $\epsilon$ in $\varepsilon^{\prime}$ that

$$
\lambda_{\epsilon} \Delta T_{j} \ll 1
$$

In this case the expected decay rates can be written

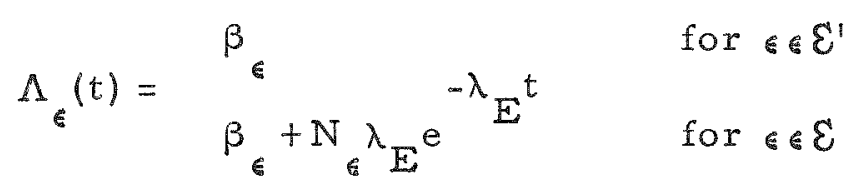

From Eq. (V.8) it follows that

$$
\begin{aligned}
& \beta_{\epsilon}^{\tau} \epsilon \quad \text { for } \& \in \mathcal{E}^{\prime} \\
& G(t-\tau, t)=\quad \beta_{\epsilon} \sigma_{\epsilon}^{T}+N_{\epsilon} \lambda_{E} E^{T} e^{-\lambda \epsilon} e^{t} \text { for } \epsilon \epsilon \mathcal{E},
\end{aligned}
$$

where we have introduced an approximation

$$
e^{\lambda_{E^{\top} \epsilon}} \approx 1+\lambda_{E^{\top} \epsilon}
$$

which must be valid in order to obtain useful data.

The expected number of gamma rays of energy E actually counted in the jth interval, Eq. $(V .9)$, can now be written in the form

$$
\left.n_{j}(E)=\int_{T_{j}}^{T_{j}+\Delta T_{j}} d t\left[\beta_{E}+N_{E} \lambda_{E} e^{-\lambda_{E} t}\right]^{\exp -\psi-\chi_{j} \lambda_{E}} e^{-\lambda_{E} t}\right\}
$$

where

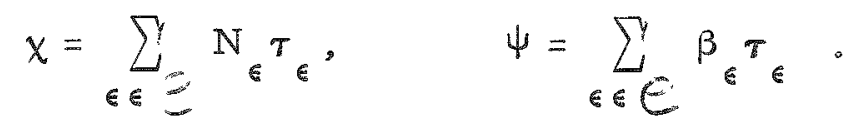


Here $\because$ is the entire observed spectrum, $i_{0} e_{0}$, the union of $\mathcal{E}$ and $\mathcal{E}^{\prime}$. The number of gamma rays of energy $E$ which would have been counted on the jth interval if the dead time of the counter were negligible is

$$
n_{j}^{0}(E)=\beta_{E} \Delta T_{j}+N_{E}\left(y_{I}-y_{2}\right)
$$

where

$$
y_{I}=e^{-\lambda_{E} T_{j}}, \quad y_{2}=y_{I} e^{-\lambda_{E} \Delta T_{j}}
$$

In terms of this notation Eq. $(V .12)$ becomes

$$
d_{j} \Delta T_{j}=\psi \Delta T_{j}+x\left(y_{I}-y_{2}\right)
$$

Equation (V.17) can be integrated to give

$\mathrm{n}_{j}(\mathrm{E})-\mathrm{b}_{j}(\mathrm{E})=\left(\mathrm{N}_{E} / \lambda_{E} X\right) \exp \left\{-\lambda_{E} \mathrm{y}_{2} x-\psi\right\}\left[1-\exp \left\{-\lambda_{E} X\left(y_{2}-y_{2}\right)\right\}\right]$,

where, by Eq. (V.10),

$$
n_{j}(E)-b_{j}(E)=\sqrt{\pi} a_{1 j} a_{3 j}
$$

Combining these equations and eliminating the quantity $\psi$ by means of the relation ( V.21) we obtain

$\sqrt{\pi} a_{1 j} a_{3 j} e^{d}=\left(N_{E} / \lambda_{E} x\right) \exp \left\{-\lambda_{E} y_{2} x+x\left(y_{I}-y_{2}\right) / \Delta T_{j}\right\}\left[1-\exp \left\{-\lambda_{E} x\left(y_{I}-y_{2}\right)\right\}\right]$.

This is the desired relation between the observed intensities, Eq. (V.23), and the "unknowns" $\lambda_{E}$ and $N_{E}$.

It is usually possible to introduce approximations which considerably simplify the relation (V.24). Since Eq. (V.19) may be 
written

$$
\lambda_{E} x\left(y_{I}-y_{2}\right)=\left(\lambda_{E} X / N_{E}\right)\left(n_{j}{ }^{0}(E)-\beta_{E} \Delta T_{j}\right)
$$

where $X / N_{E}$ is of the order of the maximum value of the resolution time $\tau_{\epsilon}$, it is easily seen that for

$$
\mathrm{n}_{\mathrm{j}}{ }^{0}(\mathrm{E}) \ll \tau_{E} / \lambda_{\mathrm{E}}
$$

Eq. (V.24) may be written

$$
\sqrt{\pi} a_{1 j} a_{3 j} e^{d}=N_{E}\left(y_{1}-y_{2}\right) e^{-\lambda_{E} y^{y_{2}}} e^{\chi\left(y_{1}-y_{2}\right) / \Delta T_{j}} .
$$

Similarly, if the average counting rate in the photopeak at energy $E$ is such that

$$
\mathrm{n}_{j}\left(\mathrm{E}_{\mathrm{j}}\right) / \Delta \mathrm{T}_{\mathrm{j}} \ll 1 / \tau_{\mathrm{E}^{2}}
$$

we have, neglecting second order and higher terms in both $n_{j} T_{E} / \Delta T_{j}$ and $\mathrm{n}_{\mathrm{j}} \lambda_{\mathrm{E}} / \mathrm{T}_{\mathrm{E}}$

$$
\sqrt{\pi a_{1 j} a_{3 j}} e^{d_{j}} e^{\lambda_{E} y_{2} x}=N_{E}\left(y_{I}-y_{2}\right)\left[1+x\left(y_{I}-y_{2}\right) / \Delta T_{j}\right] 。
$$

We have used the above procedure to measure the decay time of several radioactive nuclei. In each of these measurements the counting rates were such that the approximation (V.29) could be used with negligible error. Given a sequence of $L$ such measurements of $a_{1 j} a_{3 j}$ and $d_{j}$ for the same source, one can use the principle of least squares to obtain estimates of $\mathrm{N}_{E}$ and $\lambda_{E^{\circ}}$ The least-squares criterion is simply the minimization of $\mathrm{S}$, where 
$S=\sum_{j=1}^{L}\left\{a_{1 j} a_{3 j}-\pi^{-\frac{1}{2}} e^{-d_{j}-\lambda_{E} y_{2} x} N_{E}\left(y_{I}-y_{2}\right)\left(1+x\left(y_{I}-y_{2}\right) / \Delta T_{j} / \operatorname{var}\left(a_{1 j} a_{3 j}\right) / 。\right.\right.$

It is assumed that the uncertainties associated with the measured values $T_{j}, \Delta T_{j}$ and $d_{j}$ are negligible compared with the uncertainty in the value of $a_{1 j} a_{3 j}$ and

$$
\operatorname{var}\left(a_{1 j} c_{3 j}\right) \approx a_{1} \dot{c}^{2} \operatorname{var} a_{3}+a_{3} 2 \operatorname{var} a_{1}+2 a_{1} a_{3} \operatorname{cov}\left(a_{1}, a_{3}\right)
$$

The variances and covariances on the right-hand side of Eq. (V.31) are estimated as described in Sec.II.

Since the minimum value of $S$ in Eq. $(V .30)$ is distributed as chi-squared with $L-2$ degrees of freedom, ${ }^{13}$ it is possible to judge the validity of the error estimates in Eq. (V.31) by comparing a series of observed values of min (S) with the expectation value of this quantity. Such a comparison is shown in Table I. The agreement is seen to be quite satisfactory.

In a similar manner. maximum likelihood estimates of $\mathrm{N}_{E}$ and $\lambda_{E}$ can be calculated from Eq. (V.29) on the assumption that for each observation the number of counts in the photopeak at $E$ is distributed according to the Poisson law. The results of some measurements $^{35}$ of the decay times of radioactive nuclei are shown in Table II. In each case, the standard deviation associated with the calculated value of the half-life has been normalized to external consis tency.

TABLEI. A comparison of the observed minimum values of $S, E q$. $(V, 30)$, and the corresponding expected values $x^{2}$ for a series of deter minations of mean life.

\begin{tabular}{cc}
\hline$x^{2}$ & $\min (S)$ \\
\hline 8 & 6.2 \\
8 & 3.0 \\
7 & 1.3 \\
10 & 13.5 \\
5 & 6.3 \\
5 & 6.8 \\
9 & 2.8 \\
9 & 5.5 \\
\hline
\end{tabular}


TABLE II. Measured half-lives

\begin{tabular}{clll}
\hline $\begin{array}{c}\text { Radioactive } \\
\text { source }\end{array}$ & $\begin{array}{c}\text { Chemical } \\
\text { form }\end{array}$ & By least squares & $\frac{\text { Half-life }}{\text { By maximum likelihood }}$ \\
\hline $\mathrm{C138}$ & $\mathrm{MgCl}_{2}$ & $37.12 \pm 0.18 \mathrm{~min}$ & $37.18 \pm 0.21 \mathrm{~min}$ \\
$\mathrm{G138}$ & $\mathrm{NaCl}$ & $37.53 \pm 0.60 \mathrm{~min}$ & $37.75 \pm 0.90 \mathrm{~min}$ \\
$\mathrm{~K}_{42}$ & $\mathrm{KF}$ & $12.47 \pm 0.07 \mathrm{hr}$ & $12.42 \pm 0.06 \mathrm{hr}$ \\
$\mathrm{Na24}$ & $\mathrm{NaF}$ & $15.05 \pm 0.05 \mathrm{hr}$ & $15.04 \pm 0.05 \mathrm{hr}$ \\
\hline
\end{tabular}

Discussion and Conclusions

The experimental results presented in this section indicate that our calculated exror estimates are realistic. This means that with sufficient care an accuracy of about $0.1 \%$ can be obtained in the measure ment of gamma-ran energies in the interval from $0.5 \mathrm{Mev}$ to roughly 3.0 Mev with a scintillation counter of the type used in the above experiments. There is little doubt that the energy interval in which $0.1 \%$ measurements are feasible can be extended downward at least to $0.1 \mathrm{Mev}$. A similar analysis of mean-life data indicates that the relative intensity of a source (i.e., the product $a_{1} a_{3}$ ) can be measured with an accuracy of about $0.3 \%$ to $0.5 \%$.

In order to improve the accuracy with which energy can be determined with a scintillation spectrometer, it is obviously necessary that the relation between the energy of an incident gamma ray and the size of the resulting output pulse be determined with greater precision than we have been able to attain. As indicated by the results discussed at the end of the last subsection the inaccuracy of the interpolation formula (IV, 14) is the major source of uncertainty in energy measurements. There exist several possible sources of nonlinearity in the mechanism of light formation and transfer within the crystal. However, the observed deviations 
from linearity seem to be of the order of magnitude which might be expected from nonlinearities in the electronic equipment necessary to these measurements. Certainly this prosaic possibility should be investigated. In the determination of the absolute strength of a gammaray source, an error of $0.5 \%$ in the measured value of $a_{1} a_{3}$ is insignificant compared with the uncertainty in any calculated value of the photoefficiency of a given experimental setup.

Both the attainable accuracy and the range of applicability of these techniques for measuring mean lives deserve further investiga tion. Certainly an accuracy of about one percent is easily achieved in such measurements whenever the mean life is (1) sufficiently long that no special precautions need to be taken in the measurement of time intervals but (2) sufficiently short that a decay can be measured before drifts and other time-dependent instabilities become a source of uncer = tainty.

\section{APPENDIX。THE LEAST-SQUARES FORMALISM}

None of the standard works on statistical estimation seem to formulate the principle of least squares with sufficient generality to include all the applications considered in this report. Hence a brief outline of such a formulation is given in this appendix.

Let $\left\{x_{i}: i=1,2, \ldots, n\right\}$ denote a set of observed values of the variables $\left\{\eta_{i}: i=1,2, \ldots, n\right\}$. Suppose that these variables are related by a family of structural equations

$$
g_{K}\left(\eta_{I}, \ldots, \eta_{n}: \gamma_{I}, \ldots, \gamma_{r}\right)=0, \quad k=1,2, \ldots, \nu,
$$

which involve $r$ unknown parameters $\gamma_{a}{ }^{\circ} \operatorname{Let}\left\{c_{a}: a=1,2, \ldots, r\right\}$ be a set of estimates for the values of these $r$ parameters and let $\left\{X_{i}: i=1,2, \ldots, n\right\}$ be a set of estimates for the values of the 
variables. Let $R_{i}$ denote the $i$ th residual, $i, e$.

$$
R_{i}=x_{i}-x_{i}{ }^{2} \quad i=1,2, \ldots, n,
$$

and let $\Lambda_{i}{ }^{2}$ be the weight which is associated with the observed value $x_{1}$ so that

$$
\Lambda_{i}^{2} \propto 1 / \operatorname{var}\left(\mathrm{x}_{i}\right), \quad i=1,2, \ldots, n,
$$

where var $\left(x_{i}\right)$ is the variance of $x_{i}$. Finally let $Q$ denote the weighted sum of the squares of the $n$ residuals, $i_{0} e_{0}$

$$
Q=\sum_{i=1}^{p} \Lambda_{i}^{2} R_{i}^{2}
$$

The principle of least squares requires that the estimates $\mathrm{X}_{\mathrm{i}}$ and $\mathrm{c}_{a}$ be determined subject to the set of conditional equations

$g_{K}\left(X_{I}, \cdots, X_{n}: C_{I}, \ldots, C_{K}\right)=0, \quad K=1,2, \ldots, k$

such that the quadratic $Q$ is a minimum.

In order to carry out the least-squares prescription it is convenient to introduce the following notation:

$$
\begin{aligned}
& g_{K i}=\frac{\partial}{\partial X_{i}} g_{K}\left(X_{1}, \ldots, X_{n} ; c_{I} \ldots \ldots, c_{I}\right), K=1,2, \ldots, v ; i=1,2, \ldots, n_{2}\left(A_{0}, 6 a\right) \\
& h_{k a}=\frac{\partial}{\partial c_{a}} g_{k}\left(X_{1}, \ldots, x_{n}: c_{1}, \ldots, c_{r}\right), k=1,2, \ldots, v ; a=1,2, \ldots, x,(A, 6 b) \\
& g_{K}=g_{K}\left(x_{I}, \ldots, x_{n}: c_{1} 0, \ldots, c_{I} 0\right), \quad k=1,2, \ldots, v_{3}
\end{aligned}
$$

$$
\Delta_{a}=c_{a}^{0}-c_{a}, \quad a=1,2, \ldots, r
$$


where the $c_{a}{ }^{0}$ are the initial values of the parameters $c_{a}$. We will need the numerical values of the quantities $g_{K i}$ and $h_{K a}$ and, since these depend on the estimates which we are attempting to evaluate, the procedure is necessarily an iterative one. It is usually sufficient to use the observed values $x_{i}$ for $x_{i}$ in the initial iteration and we as sume that values $c_{a}^{0}$ can somehow be found such that the conditions necessary for the stability of the iterative sequence are satisfied.

Expansion of Eq. (A.6c) in a Taylor series about the esti= mates $X_{i}$ and $c_{a}$ leads to

$$
g_{k 0}=\sum_{i=1}^{n} g_{k i} R_{i}+\sum_{a=1}^{r} h_{k a} \Delta_{a} \quad k=1,2, \ldots, \nu,(A, 7)
$$

where the condicions expressed by the set of equations (A.5) have been used and residuals of order two and higher have been neglected. Denoting by of the variation of a function $f$ with respect to the estimates, we obtain from Eqs, (A.4) and (A,7)

$$
\frac{1}{2} \delta Q=\sum_{i=1}^{n} \Lambda_{i}^{2} R_{i} \delta R_{i}=0
$$

and

$$
\delta g_{\kappa}=\sum_{i=1}^{n} g_{\kappa^{i}} \delta R_{i}+\sum_{a=1}^{r} h_{\kappa a} \delta \Delta_{a}=0, \quad k=1,2, \ldots, \nu,
$$

We multiply the $\kappa^{\text {th }}$ equation of the set $(A .9)$ by the arbitrary Lagrange multiplier $\ell_{K}$ and add the resulting $v$ equations to Eq. (A.8) to obtain

$$
\sum_{i=1}^{n}\left\lceil\Lambda_{i}{ }^{2} R_{i}+\sum_{\kappa=1}^{\nu} \ell_{K} g_{K i}\right\rceil \delta R_{i}+\sum_{a=1}^{r} \sum_{K=1}^{\nu} \ell_{K} h_{K a} \Delta_{a}=0 .
$$

This equation contains $n+r$ variations of which only $n+r-v$ are independent because of the conditional equations (A.5). We choose values for the $\ell_{K}$ such that the coefficients of $v$ of the variations in $(A, 10)$ vanish. The 
remaining $n+r-v$ variations are independent so that, with this choice of the $\ell_{K}$, all of the coefficients in Eq. (A.10) vanish and we can write

$$
\mathrm{R}_{i}=\Lambda_{\mathrm{i}}-2 \sum_{\kappa=1}^{\nu} \ell_{\kappa} \mathrm{g}_{\kappa^{\mathrm{i}}} \quad \mathrm{i}=1,2, \ldots, \mathrm{n}
$$

and

$$
0=\sum_{\kappa=1}^{\nu} \ell_{K}{ }_{K} a^{\prime} \quad a=1,2, \ldots, r_{0}
$$

The substitution of the right-hand side of $E q .(A, 11)$ for the $R_{i}$ in $E q .(A, 7)$ gives

$$
g_{\kappa 0}=\sum_{i=1}^{n} g_{K^{i}} \Lambda_{i}{ }^{-2} \sum_{\sigma=1}^{\nu} \ell_{\sigma^{\prime}} g_{\sigma_{i}}+\sum_{a=1}^{r} h_{K^{a}} \Delta_{a}, \quad \kappa=1,2, \ldots, v 。
$$

The $r+v$ equations (A.12) and (A.13) are the normal equations for the $v+r$ "unknowns" $\Delta a$ and $\ell_{\kappa}$.

At this point it is convenient to define (a) the $v \times v$ symmetric matrix $\mathcal{L}$, the components of which are

$$
\mathcal{L}_{\kappa \sigma}=\sum_{i=1}^{n} g_{\kappa i} \Lambda_{i}^{-2} g_{\sigma i}
$$

(b) the $\nu \times r$ matrix $H$, where

$$
H_{\kappa^{a}}=h_{\kappa^{a}}
$$

(c) the $(v+r)$-dimensional column vector 9 , where

$$
\begin{aligned}
& g_{t}{ }^{g_{t 0}, \quad t=1,2, \ldots, v,} \\
& \text { o, } \quad t=v+1, v+2, \ldots, v+r \text {; }
\end{aligned}
$$


(d) and the $(v+r)$-dimensional column vector, where

$$
\begin{array}{ll}
l_{t}, & t=1,2, \ldots, v, \\
\Delta_{a^{\prime}} & t=v+a ; a=1,2, \ldots, r .
\end{array}
$$

The normal equations (A.13) and (A.14) can be written in terms of these matrices as

$$
Y=\mathrm{Q}
$$

where $J$ is $a(v+r) \times(v+r)$ matrix

$$
J=\left|\begin{array}{cc}
f & H \\
\tilde{H} & 0
\end{array}\right|
$$

Here $\widetilde{H}$ is the transpose of the matrix $H$ and 0 is the $r \times x$ null matrix.

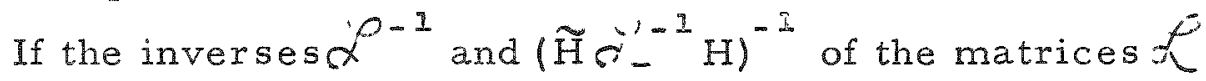
and $\tilde{\mathrm{H}} \mathcal{X}^{-1} \mathrm{H}$ exist, the left inverse $\mathrm{J}^{-1}$ of the matrix $\mathrm{J}$ exists and is given by

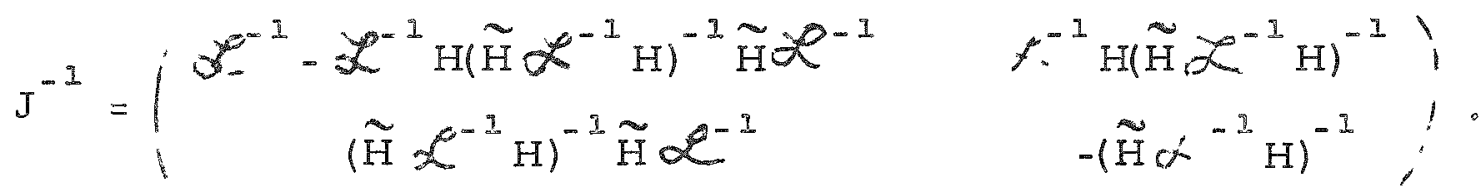

The solution of Eq. (A.18) for the vector $U$, which contains as its components the values of the Lagrange multipliers $\ell_{K}$ and of the iterates $c_{a}$, can be written as

$$
i=J^{-2} y
$$

In component form this matrix relation gives

$$
\begin{aligned}
& \ell_{\sigma}=\sum_{t=1}^{\nu}\left(J^{-I}\right) g_{t 0} \\
& \left.=\sum_{t=1}^{\nu} \cdot\left(f^{-1}\right)_{\sigma t}-\sum_{\kappa, \mu=1}^{\nu} \sum_{a, \beta=1}^{r}\left(\mathcal{L}^{-1}\right)_{\sigma \kappa} \mathrm{H}_{\kappa \mathrm{a}}\left[\left(\tilde{\mathrm{H}} \mathcal{X}^{-1} \mathrm{H}\right)^{-1}\right]_{\alpha \beta} \mathrm{H}_{\mu \beta}\left(\mathcal{X}^{-1}\right)_{\mu t}\right\}_{\mathrm{t} 0}^{\left(\mathrm{A}_{0} 22\right)}
\end{aligned}
$$


and

$$
\begin{aligned}
\Delta_{a} & =\sum_{t=1}^{v}\left(J^{-1}\right)_{v+a, t} g_{t 0} \\
& =\sum_{t, \kappa=1}^{\nu} \sum_{\beta=1}^{r}\left[\left(\widetilde{H} \mathscr{L}^{-1} \mathrm{H}\right)^{-1}\right]_{a \beta} H_{\kappa \beta}\left(\mathcal{X}_{-1}^{-1}\right)_{\kappa} g_{t 0},
\end{aligned}
$$

where $\sigma=1,2, \ldots, v$ and $a=1,2, \ldots, r$.

The estimates $X_{i}$ are obtained from Eq. (A. 11) with the values of the Lagrange multipliers obtained from Eq. (A,22). The estimates $c_{a}$ are given as the solutions of Eq. (A.23). Obviously the values of $\mathrm{X}_{i}$ and $\mathrm{C}_{a}$ can be used in an iterative manner in the evaluation of the derivatives defined by Eqs. (A.6a) and (A.6b). Similarly, the values $c_{a}$ can be used for the initial values $c_{a}^{0}$ in Eq. (A.6c) at any stage in the iteration process.

The values of the elements of the error matrix associated with the joint probability distribution of the $c_{a}$ can be estimated as follows. Since the set of equations (A.23) remains valid for any value of $c_{a}{ }^{0}$, we can write for $a=1,2, \ldots, r$

$\gamma_{a}-c=\sum_{t}^{\nu} \sum_{\kappa=1}^{r}\left[\left(\tilde{H} \mathcal{L}^{-1} H\right)^{-1}\right]_{a \beta} H_{\kappa \beta}\left(\mathcal{L}^{-1}\right)_{\kappa t} g_{t}\left(x_{I} \ldots \ldots x_{n}: \gamma_{I}, \ldots, \gamma_{1 r}\right)$,

where the $\gamma_{a}$ are the "true values" of the estimates $c_{a}$. If the notation $\left\langle g_{t}\right\rangle$ indicates the average value of $g_{t}\left(x_{1}, \ldots, x_{n}: \gamma_{1}, \ldots, \gamma_{r}\right)$ for a long series of measurements of the $n$-tuple $\left(x_{1}, \ldots, x_{n}\right)$, then to a first approximation its value is

$$
\left\langle\mathrm{g}_{\mathrm{t}}\right\rangle=0, \quad \mathrm{t}=1,2, \ldots, v,
$$

and

$$
\begin{aligned}
\left\langle g_{t} g_{t^{\prime}}\right\rangle & =\sum_{i, j=1}^{n} g_{t i^{\prime} g^{\prime} j}\left\langle\left(x_{i}-\eta_{i}\right)\left(x_{j}-\eta_{j}\right)\right\rangle \\
& =\sum_{i=1}^{n} g_{t i} g_{t^{\prime} i} \Lambda_{i}^{-2}=\alpha_{t t^{\prime}} \quad t, t^{\prime}=1,2, \ldots, v,
\end{aligned}
$$


From Eq. (A.24) it follows that

$$
\begin{aligned}
& \left\langle\left(\gamma_{a}-c_{a}\right)\left(\gamma_{\beta}-c_{\beta}\right)\right\rangle=\sum_{t, k_{0}, t^{\prime}, k^{\prime},=1}^{v} \sum_{\epsilon, \epsilon^{\prime}=1}^{r}\left[\left(\tilde{H} \mathcal{L}^{-1} H\right)^{-1}\right]_{a \in}\left[\left(\tilde{H} \mathcal{L}^{-1} H\right)^{-1}\right]_{\beta \in} \\
& \times H_{K \in} H_{K^{\prime} \epsilon^{\prime}}\left(\mathcal{L}^{3}\right)_{K t}\left(\mathcal{L}^{-3}\right)_{K^{\prime} t^{\prime}}\left\langle g_{t} g_{t}{ }^{\prime}\right\rangle \\
& =\left[\left(\tilde{\mathrm{H}} \mathscr{\mathcal { L }}^{-1} \mathrm{H}\right)^{-1}\right]_{a \beta^{0}} \quad a, \beta=1,2, \ldots, r \text {. }
\end{aligned}
$$

Thus the varlance and covariance of the estimates $c_{a}$ are

$$
\operatorname{var}\left(c_{a}\right)=\left[\left(\tilde{H} \mathcal{L}^{-1} H\right)^{-1}\right]_{a a^{\prime}} \quad a=1,2, \ldots, r
$$

and

$$
\operatorname{cov}\left(c_{a}, c_{\beta}\right)=\left[\left(\tilde{\mathrm{H}} \rho^{2}\right)^{-1}\right]_{a \beta^{\prime}} \quad a, \beta=1,2, \ldots, r ; a \neq \beta \text {. }
$$

As an example of the application of this formalism to problems of the type considered in this report, consider the set of $\mathrm{m}$ observed pairs $\left\{\left(x_{K}{ }_{k} x_{K}+m\right): K=1,2, \ldots \circ, m\right\}$, where the "true value" $\left(\eta_{K}, \eta_{K}+\mathrm{m}\right)$ of the $\kappa^{t h}$ pair satisfies a structural equation of the form

$$
g_{k}=\eta_{K}-\gamma_{1}-\gamma_{2} \eta_{k+m}=0, \quad k=1,2, \ldots, m_{0}\left(A_{0} 29\right)
$$

The problem is to obtain estimates $c_{2}$ and $c_{2}$ of the values of the parameters $\gamma_{1}$ and $\gamma_{2}$ in the structural equations from the observed data. This is obviously a special case of the "two-error-problem" which was introduced in Sec。IV.

From Eqs. (A.6a), (A.6B), and $(A, 6 C)$ we obtain

$$
\begin{aligned}
& \mathrm{g}_{K^{i}}=\delta_{K^{i}}-c_{2} \delta_{K}+\mathrm{mi} \\
& \mathrm{h}_{K^{1}}=-1, \\
& \mathrm{~h}_{K^{2}}=-\mathrm{x}_{\kappa+\mathrm{m}},
\end{aligned}
$$


and

$$
g_{K 0}=x_{K},
$$

where $\kappa$ and $i$ take on all integral values from 1 to $m$. For the initial iteration we have set $c_{1}{ }^{0}$ and $c_{2}{ }^{0}$ equal to zero and have used the observed values $\mathrm{x}_{\kappa+\mathrm{m}}$ for the estimates $\mathrm{X}_{\kappa+\mathrm{m}}$. The residuals calculated by Eq. (A. 11) are

$$
\begin{array}{ll}
R_{i}=l_{i} \Lambda_{i}^{-2} & i=1,2, \ldots, m, \\
-c_{2} \ell_{i}-m_{i}^{-2}, & i=m+1, m+2, \ldots, 2 m,
\end{array}
$$

where

$$
\Lambda_{i}^{-2}=\operatorname{var}\left(x_{i}\right), \quad i=1,2, \ldots, 2 m
$$

The equations (A. 13) now become

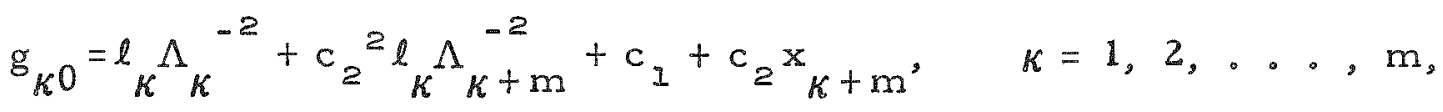

so that

$\ell_{K}=\left(g_{\kappa}-c_{I}-c_{2} x_{K+m}\right) /\left(\Lambda_{K}{ }^{-2}+c_{2}{ }^{2} \Lambda_{\kappa+m}^{-2}\right), k=1,2, \ldots, m$

Finally from Eqs. (A. 12) we obtain the normal equations for the estimates $c_{1}$ and $c_{2}$. These are

$$
\sum_{k=1}^{m}\left[\left(x_{k}-c_{1}-c_{2} x_{k+m}\right) /\left(\Lambda_{k}{ }^{-2}+c_{2}{ }^{2} \Lambda_{k+m}^{-2}\right)\right]=0
$$

and

$$
\sum_{\kappa=1}^{m}\left[x_{\kappa}+m\left(x_{k}-c_{1}-c_{2} x_{k}+m\right) /\left(\Lambda_{\kappa}^{-2}+c_{2}^{2} \Lambda_{\kappa+m}^{-2}\right)\right]=0
$$


The values $c_{1}$ and $c_{2}$ obtained as solutions of these equations can be used for $c_{1}^{0}$ and $c_{2}{ }^{0}$ and the estimates $X_{K}$ and $X_{K+m}$ obtained from Eq. (A.11) can be used for the evaluation of the derivatives $\mathrm{h}_{\mathrm{Ka}}$ in the next iteration. It is interesting to note that the normal equations (A.35a) and (A. 35b) are the conditions for the minimization of a quadratic $S$ of the form

$$
S=\sum_{\kappa=1}^{m}\left(x_{k}-c_{1}-c_{2} x_{k}+m\right)^{2} /\left(\Lambda_{k}^{-2}+c_{2}{ }^{2} \Lambda_{k+m}^{-2}\right)
$$

provided the denominator is considered constant in the variation of $S$ with respect to the estimate $\mathrm{C}_{2}$. 


\section{REEERENCES}

1

C. C. Trail and Sol Raboy, Rev. Sci. Instr. 30, 425 (1959).

${ }^{2} R_{\text {. W. Schumann and J.P. McMahon, Rev. Sci.Instr. 27, } 675}$ $(1956)$.

${ }^{3}$ E. Breitenberger, Prog. Nuclear Phys.4, 56 (1958). This article contains numerous references to other work on this subject.

${ }^{4}$ F. Seitz and D.W. Mueller, Phys,Rev。78, 605 (1950).

${ }^{5}$ P. M. Woodward, Proc.Cambridge Phil。Soc.44, 404 (1948)。

${ }^{6}$ M. G. Kendall and A. Stuart, The Advanced Theory of Statistics (Hafner Publishing Company, New York, 1958, Vol。1, p。109.

7 A. Bisi and L. Zappa, Nuclear Instr, and Methods 3, 17 (1958).

${ }^{8}$ C. M. Davisson and L.A. Beach, U.S. Naval Research Laboratory Report NRL-5408, December 31, 1959 。

J. E. Monahan, S.Raboy, and C.C.Trail, Phys。Rev。123, 1373 (1961).

${ }^{10}$ G。 T. Wright, Phys。Rev。 96, 569 (1954)。

${ }^{12}$ Y. A. Nemilov, I. I. Lomonosov, A. No Pisarevskii, L。D.

Soshin, and N.D. Teterin, Bull. Acad。Sci。U.S.S.R。23, (2) (1959).

12 A. Wald, Ann。 Math. Statistics 11, 284(1940).

${ }^{13}$ W. E。 Deming, J.Am。 Stat,Assoc。29, 372 (1934)。

14 L.I.Schiff, Phys.Rev。50, 88 (1936).

I5 J.E. Monahan, S.Raboy, and C.C.Trail, to be published. 